\title{
Catalytic Conjugate Addition of Allyl Groups to Styryl-Activated Enones
}

\author{
Joshua D. Sieber, Shubin Liu' ${ }^{1}$, and James P. Morken ${ }^{*}$ \\ Department of Chemistry, Merkert Chemistry Center, Boston College, Chestnut Hill, MA 02467 \\ ${ }^{1}$ Scientific Computing Center, University of North Carolina, Chapel Hill, NC 27599
}

\section{Supplementary Material}

General. Melting points were determined using a Mel-Temp II melting point apparatus and are uncorrected. ${ }^{1} \mathrm{H}$ NMR spectra were recorded on Bruker DRX 300 or $400 \mathrm{MHz}$ spectrometers or a Gemini-400 (400 MHz) spectrometer. Chemical shifts are reported in ppm from tetramethylsilane with the solvent resonance as the internal standard $\left(\mathrm{CDCl}_{3}: 7.24 \mathrm{ppm}\right)$. Data are reported as follows: chemical shift, integration, multiplicity ( $\mathrm{s}=$ singlet, $\mathrm{d}=$ doublet, $\mathrm{t}=$ triplet, $\mathrm{q}=$ quartet, $\mathrm{p}=$ pentet, $\mathrm{h}=$ hextet, $\mathrm{br}=$ broad, $\mathrm{m}=$ multiplet), and coupling constants $(\mathrm{Hz}) .{ }^{13} \mathrm{C}$ NMR was recorded using a Bruker $400 \mathrm{MHz}(100 \mathrm{MHz})$ instument, a Gemini-400 (100 MHz) instrument, or a Gemini-500 (125 MHz) instrument with complete proton decoupling. Chemical shifts are reported in ppm from tetramethylsilane with the solvent as the internal standard $\left(\mathrm{CDCl}_{3}\right.$ : $77.0 \mathrm{ppm}$ ). Low-resolution mass spectrometry was performed by the University of North Carolina, Department of Chemistry Mass Spectrometry Facility. Infrared (IR) spectra were obtained on a Nicolet 560 Magna-FTIR.

Liquid chromatography was performed using forced flow (flash chromatography) on silica gel $\left(\mathrm{SiO}_{2}, 40-63\right.$ $\mu \mathrm{m})$ purchased from SiliCycle. Thin layer chromatography was performed on $250 \mu \mathrm{m}$ silica gel plates from EMD Chemicals Inc. Visualization was achieved using UV light, phosphomolybdic acid in ethanol, or potassium permanganate in water, each followed by heating.

Analytical supercritical fluid chromatography (SFC) was performed on a Berger Instruments supercritical chromatograph equipped with an Alcott autosampler and a Knauer UV detector.

All reactions were conducted in oven or flame dried glassware under an inert atmosphere of nitrogen or argon. Anhydrous THF, used in reactions that were prepared in a dry-box, was purchased from Aldrich Chemical Company. For all other reactions prepared outside of a dry-box, THF that was freshly distilled from Na metal and benzophenone was used. Tris(dibenzylideneacetone)dipalladium, Bis(1,5-cyclooctadiene)nickel, and tricyclohexylphosphine were purchased from Strem Chemical Company. Allylboronic acid pinacol ester was purchased from Aldrich Chemical Company and distilled through a 6 inch Vigreux column $\left(58-62{ }^{\circ} \mathrm{C}\right.$ at 20 torr) and stored in the freezer under Ar. The styryl-activated substrates were synthesized by addition of the desired vinyl lithium reagent, prepared by Li-halogen exchange of the corresponding vinyl iodide or bromide, to $N$-methoxy- $N$-methylcinnamide at low temperature and is described below. $N$-Methoxy- $N$-methylcinnamide was synthesized according to the literature (Hiyama, T.; Reddy, G. B.; Minami, T.; Hanamoto, T. Bull. Chem. Soc. Jpn. 1995, 68, 350.). 1-Iododheptene was synthesized from 1-heptyne via hydroalumination with DIBAL followed by iodination (Stille, J. K.; Simpson, J. H. J. Am. Chem. Soc. 1987, 109, 2138. Trost, B. M.; Rudd, M. T. Org. Lett. 2003, 5, 4599.). 2-Iodovinylcyclohexane and 1-iodo-3,3-dimethyl-1-butene were synthesized via hydroboration of cyclohexylacetylene or t-butylacetylene, respectively, followed by iodination (Brown, H. C.; Hamaoka, T. Ravindran, N.; Subrahmanyam, C.; Somayaji, V.; Bhat, N. G. J. Org. Chem. 1989, 54, 6075. Gagnon, D.; Lauzon, S.; Godbout, C.; Spino, C. Org. Lett. 2005, 7, 4769.). Vinyl iodides bearing pendant TBSprotected alcohols were synthesized via hydrozirconation of the TBS-protected propargyl or homo propargyl alcohol with the Schwartz reagent, followed by iodination (Germain, J.; Deslongchamps, P. J. Org. Chem. 2002, 67, 5269.). Vinyl bromides were purchased from Aldrich Chemical Company and used without further 
purification. All other reagents were purchased from either Fisher or Aldrich Chemical Companies and used directly.

\section{Representative procedure for the synthesis of styryl-activated substrates.}<smiles>[R]C=CC(=O)C=Cc1ccccc1</smiles>

Method A, from the vinyl iodide: To $2.25 \mathrm{~g}(10.0 \mathrm{mmol})$ of 1 -iodoheptene in $10 \mathrm{~mL}$ of THF at $-78{ }^{\circ} \mathrm{C}$ was added $4.2 \mathrm{~mL}$ (10 mmol) of a $2.4 \mathrm{M}$ solution of $n$-BuLi in hexane dropwise. This solution was stirred for $30 \mathrm{~min}$ at $-78{ }^{\circ} \mathrm{C}$ and then transferred dropwise via canula to a solution of $0.965 \mathrm{~g}$ (5.00 mmol) of $N$-methoxy- $N$ methylcinnamide in $50 \mathrm{~mL}$ of THF at $-78{ }^{\circ} \mathrm{C}$. After complete addition, TLC analysis showed complete consumption of the starting material after $15 \mathrm{~min}$ at $-78{ }^{\circ} \mathrm{C}$, so the reaction was subsequently quenched with satd. $\mathrm{NH}_{4} \mathrm{Cl}_{(a q)}$. The crude reaction was transferred to a separatory funnel with $1 \mathrm{M} \mathrm{HCl}$ and $\mathrm{CH}_{2} \mathrm{Cl}_{2}$. The organic layer was collected after shaking, and the aqueous layer was extracted with $\mathrm{CH}_{2} \mathrm{Cl}_{2}(1 \mathrm{x})$. The combined organics were dried over $\mathrm{Na}_{2} \mathrm{SO}_{4}$ and concentrated using reduced pressure. Silica gel chromatography (hexanes/EtOAc) of the crude mixture afforded $1.0 \mathrm{~g}(91 \%)$ of $(1 E, 4 E)-1$-phenyldeca-1,4-dien-3-one as a yellow oil. Spectral data was consistent with the literature (Tsuge, O.; Kanemasa, S.; Nakagawa, N.; Suga, H. Bull. Chem. Soc. Jpn. 1987, 69, 4091.).

Method B, from the vinyl bromide: To $1.05 \mathrm{~g}$ of (E)-1-bromopropene in $16 \mathrm{~mL}$ of THF at $-78{ }^{\circ} \mathrm{C}$ was added $10.8 \mathrm{~mL}(17 \mathrm{mmol})$ of a $1.6 \mathrm{M}$ solution of $t$-BuLi in pentane dropwise. This solution was stirred at this temperature for $30 \mathrm{~min}$ and then transferred at $\sim 1 \mathrm{drop} / \mathrm{s}$ via canula to a solution of $0.832 \mathrm{~g}$ of $N$-methoxy- $N$ methylcinnamide in $33 \mathrm{~mL}$ of THF at $-78{ }^{\circ} \mathrm{C}$ (addition of the vinyllithium at faster rates gave lower yields). After complete addition, TLC analysis showed complete consumption of the starting material after 15 min at $78{ }^{\circ} \mathrm{C}$, so the reaction was subsequently quenched with satd. $\mathrm{NH}_{4} \mathrm{Cl}_{(a q)}$. The crude reaction was transferred to a separatory funnel with $1 \mathrm{M} \mathrm{HCl}$ and $\mathrm{Et}_{2} \mathrm{O}$. The organic layer was collected after shaking, and the aqueous layer was extracted with $\mathrm{Et}_{2} \mathrm{O}(1 \mathrm{x})$. The combined organics were washed with $\mathrm{H}_{2} \mathrm{O}$ then brine and finally dried over $\mathrm{Na}_{2} \mathrm{SO}_{4}$ and concentrated using reduced pressure. Silica gel chromatography (hexanes/EtOAc) of the crude mixture afforded $0.571 \mathrm{~g}(76 \%)$ of (1E, 4E)-1-phenylhexa-1,4-dien-3-one as a yellow oil.

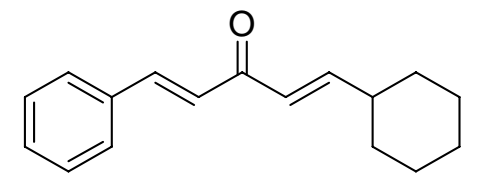

(1E, 4E)-1-cyclohexyl-5-phenylpenta-1,4-dien-3-one. Prepared using Method A in $96 \%$ yield. A yellow solid. mp $42-46{ }^{\circ} \mathrm{C} . \mathrm{R}_{f}=0.20$ (12:1 Hexanes:EtOAc); IR (KBr): 2924 (m), 2847 (m), 1806 (w), 1670 (s), 1627 (s), 1592 (s), 1441 (s), 1332 (s), $1278(\mathrm{~m}), 1184(\mathrm{~m}) \mathrm{cm}^{-1}$; ${ }^{1} \mathrm{H}$ NMR: $\delta 7.62(1 \mathrm{H}, \mathrm{d}, J=16 \mathrm{~Hz}), 7.55(2 \mathrm{H}, \mathrm{m})$, 7.30-7.42 (3H, m), 6.97 (1H, d, $J=16 \mathrm{~Hz}), 6.93$ (1H, dd, $J=16 \mathrm{~Hz}, J=6.8 \mathrm{~Hz}), 6.37(1 \mathrm{H}, \mathrm{dd}, J=14 \mathrm{~Hz}, J=$ $1.2 \mathrm{~Hz}), 2.19(1 \mathrm{H}, \mathrm{m}), 1.61-1.88(5 \mathrm{H}, \mathrm{m}) 1.10-1.40(5 \mathrm{H}, \mathrm{m}) ;{ }^{13} \mathrm{C} \mathrm{NMR}: \delta 189.6,153.2,142.8,134.8,130.3$, 128.9, 128.2, 126.8, 124.8, 40.87, 31.80, 25.90, 25.71. LRMS (ESI+) Calc’d for $\mathrm{C}_{17} \mathrm{H}_{20} \mathrm{O}(\mathrm{M}+\mathrm{Na})^{+}: 263.1$ Found $(\mathrm{M}+\mathrm{Na})^{+}$: 263.2 . 


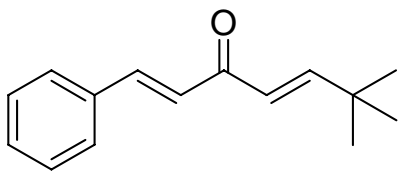

(1E, 4E)-6,6-dimethyl-1-phenylhepta-1,4-dien-3-one. Prepared using Method A in $86 \%$ yield. An off-white solid. $\mathrm{mp} 60-64{ }^{\circ} \mathrm{C} . \mathrm{R}_{f}=0.30$ (12:1 Hexanes:EtOAc); IR (KBr): $3060(\mathrm{w}), 3017(\mathrm{w}), 2948(\mathrm{~m}), 2862(\mathrm{w}), 1950(\mathrm{w}), 1880(\mathrm{w}), 1658(\mathrm{~s}), 1588$ (s), 1449 (m), 1324 (s), 1208 (s), 1165 (s) cm ${ }^{-1} ;{ }^{1} \mathrm{H}$ NMR: $\delta 7.63(1 \mathrm{H}, \mathrm{d}, J=16 \mathrm{~Hz})$, $7.56(2 \mathrm{H}, \mathrm{m}), 7.37$ (3H, m), $6.98(2 \mathrm{H}, \mathrm{d}, J=16 \mathrm{~Hz}), 6.32(1 \mathrm{H}, \mathrm{d}, J=16 \mathrm{~Hz}), 1.12(9 \mathrm{H}, \mathrm{s}) ;{ }^{13} \mathrm{C}$ NMR: $\delta$ 189.7, 157.9, 142.9, 134.8, 130.3, 128.9, 128.3, 124.9, 124.5, 33.97, 28.72. LRMS (ESI+) Calc'd for $\mathrm{C}_{15} \mathrm{H}_{18} \mathrm{O}(\mathrm{M}+$ $\mathrm{Na})^{+}:$237.1 Found $(\mathrm{M}+\mathrm{Na})^{+}: 237.1$.

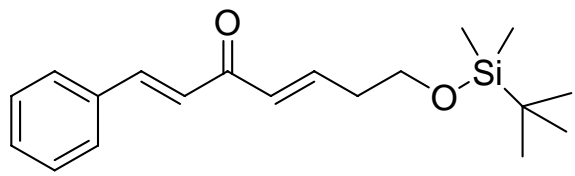

(1E, 4E)-7-(t-butyldimethylsilyloxy)-1-phenylhepta-1,4-dien-3-one. Prepared using Method A in 87\% yield. A yellow oil. $\mathrm{R}_{f}=0.19$ (12:1 Hexanes:EtOAc); IR (neat): 3029 (w), 2952 (s), 2854 (s), 1950 (w), 1802 (w), 1662 (s), 1631 (s), $1596(\mathrm{~s}), 1472(\mathrm{~m}), 1336(\mathrm{~m}), 1254(\mathrm{~s}), 1185(\mathrm{~m})$ $\mathrm{cm}^{-1}$; ${ }^{1} \mathrm{H}$ NMR: $\delta 7.63(1 \mathrm{H}, \mathrm{d}, J=16 \mathrm{~Hz}), 7.55(2 \mathrm{H}, \mathrm{m}), 7.38(3 \mathrm{H}, \mathrm{m}), 6.98(1 \mathrm{H}, \mathrm{dt}, J=16 \mathrm{~Hz}, J=8.6 \mathrm{~Hz})$, $6.96(1 \mathrm{H}, \mathrm{d}, J=16 \mathrm{~Hz}), 3.61(1 \mathrm{H}, \mathrm{dt}, J=16 \mathrm{~Hz}, J=1.2 \mathrm{~Hz}), 3.76(2 \mathrm{H}, \mathrm{t}, J=6.4 \mathrm{~Hz}) 3.08(2 \mathrm{H}, \mathrm{dq}, J=7.2 \mathrm{~Hz}, J$ $=1.6 \mathrm{~Hz}), 0.88(9 \mathrm{H}, \mathrm{s}), 0.050(3 \mathrm{H}, \mathrm{s}), 0.042(3 \mathrm{H}, \mathrm{s}) ;{ }^{13} \mathrm{C}$ NMR: $\delta 189.1,144.7,143.1,134.8,130.8,130.3$, 128.9, 128.2, 124.6, 61.60, 36.15, 25.86, 18.28, -5.33. LRMS (ESI+) Calc'd for $\mathrm{C}_{19} \mathrm{H}_{28} \mathrm{O}_{2} \mathrm{Si}(\mathrm{M}+\mathrm{Na})^{+}: 339.2$ Found $(\mathrm{M}+\mathrm{Na})^{+}: 339.2$.

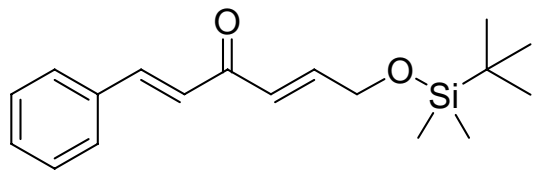

$(1 E$,

4E)-6-(t-butyldimethylsilyloxy)-1-phenylhexa-1,4-dien-3-one. Prepared using Method A in 60\% yield. A yellow oil. $\mathrm{R}_{f}=0.20(12: 1$ Hexanes:EtOAc); IR (neat): 3060 (w), 2955 (s), 2932 (s), 2854 (s), 1953 (w), 1806 (w), 1666 (s), 1634 (s), 1592 (s), 1449 (s), 1332 (s), 1254 (s), 1138 (s) $\mathrm{cm}^{-1}$; ${ }^{1} \mathrm{H}$ NMR: $\delta 7.64(1 \mathrm{H}, \mathrm{d}, J=16 \mathrm{~Hz}), 7.56(2 \mathrm{H}, \mathrm{m}), 7.38(3 \mathrm{H}, \mathrm{m}), 7.02(1 \mathrm{H}, \mathrm{dt}, J=15 \mathrm{~Hz}, J=3.6 \mathrm{~Hz})$, $6.95(1 \mathrm{H}, \mathrm{d}, J=16 \mathrm{~Hz}), 6.73(1 \mathrm{H}, \mathrm{dt}, J=15 \mathrm{~Hz}, J=2.0 \mathrm{~Hz}), 4.41(2 \mathrm{H}, \mathrm{m}), 0.94(9 \mathrm{H}, \mathrm{s}), 0.10(6 \mathrm{H}, \mathrm{s}) ;{ }^{13} \mathrm{C} \mathrm{NMR}$ : $\delta$ 189.1, 146.1, 143.4, 134.7, 130.4, 128.9, 128.3, 126.5, 125.3, 62.52, 25.86, 18.37, -5.41. LRMS (ESI+) Calc'd for $\mathrm{C}_{18} \mathrm{H}_{26} \mathrm{O}_{2} \mathrm{Si}(\mathrm{M}+\mathrm{H})^{+}$: 303.2 Found $(\mathrm{M}+\mathrm{H})^{+}$: 303.2 .

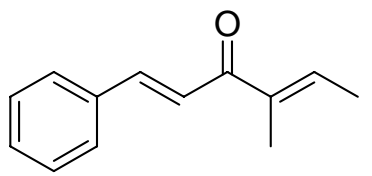

(1E, 4E)-4-methyl-1-phenylhexa-1,4-dien-3-one. Prepared using Method B in 53\% yield. A yellow oil. $\mathrm{R}_{f}=0.19$ (14:1 Hexanes:EtOAc); IR (neat): 3060 (m), 2920 (m), 1953 (w), 1887 (w), 1654 (s), 1600 (s), 1449 (s), 1328 (s), 1300 (s), 1223 (s) cm ${ }^{-1}$; ${ }^{1} \mathrm{H}$ NMR: $\delta 7.59(1 \mathrm{H}, \mathrm{d}, J=16 \mathrm{~Hz}), 7.55(2 \mathrm{H}, \mathrm{m}), 7.37(3 \mathrm{H}, \mathrm{m}), 7.28(1 \mathrm{H}, \mathrm{d}, J=16 \mathrm{~Hz})$, $6.82(1 \mathrm{H}, \mathrm{q}, J=6.0 \mathrm{~Hz}), 1.89(3 \mathrm{H}, \mathrm{d}, J=6.0 \mathrm{~Hz}), 1.88(3 \mathrm{H}, \mathrm{s}) ;{ }^{13} \mathrm{C}$ NMR: $\delta 191.7,142.5,139.3,137.4,135.2$, 129.9, 128.8, 128.1, 121.7, 14.80, 11.55. LRMS (ESI+) Calc'd for $\mathrm{C}_{13} \mathrm{H}_{14} \mathrm{O}(\mathrm{M}+\mathrm{Na})^{+}$: 209.1 Found (M + $\mathrm{Na})^{+}: 209.1$.

\section{Procedure for the catalytic conjugate allylation of dibenzylidene acetone, chalcone, and benzylidene} acetone (Scheme 1).

The procedure for dibenzylidene acetone is representative. An oven-dried $20 \mathrm{~mL}$ vial equipped with a magnetic stir-bar was charged with $4.9 \mathrm{mg}(0.0053 \mathrm{mmol})$ of tris(dibenzylideneacetone)dipalladium, $3.6 \mathrm{mg}$ $(0.013 \mathrm{mmol})$ of tricyclohexylphosphine, and $1.42 \mathrm{~mL}$ of THF in a dry-box under an argon atmosphere. The vial was capped and stirred for $45 \mathrm{~min}$. Next, $39.4 \mathrm{mg}(0.234 \mathrm{mmol})$ of allylboronic acid pinacol ester was added followed by $50.0 \mathrm{mg}(0.213 \mathrm{mmol})$ of dibenzylidene acetone. The vial was capped, taped with electrical 
tape, removed from the dry-box, and allowed to stir at ambient temperature for the time indicated in Scheme 1. After this time period, water was added and the mixture transferred to a separatory funnel with $\mathrm{CH}_{2} \mathrm{Cl}_{2}$. After gently swirling the layers (to avoid formation of emulsions), the organic layer was collected and the aqueous layer washed with $\mathrm{CH}_{2} \mathrm{Cl}_{2}(1 \mathrm{x})$. The combined organic layers were dried with $\mathrm{Na}_{2} \mathrm{SO}_{4}$, and volatiles were removed under reduced pressure. Silica gel chromatography (hexanes/EtOAc) afforded 50.4 mg (80\%) of 1,5diphenyl-1,7-octadien-3-one as a white solid whose spectral data was consistent with the literature (Mandal, S. K.; Amin, Sk. R.; Crowe, W. E. J. Am. Chem. Soc. 2001, 123, 6457.).

\section{Procedure for the catalyst survey in the conjugate allylation (Table 1).}

Procedure using $\mathbf{P d}_{\mathbf{2}}(\mathbf{d b a})_{3}$ : An oven-dried 2-dram vial equipped with a magnetic stir-bar was charged with $2.3 \mathrm{mg}(0.0025 \mathrm{mmol})$ of tris(dibenzylideneacetone)dipalladium, $0.0060 \mathrm{mmol}$ of phosphine ligand, and $0.20 \mathrm{~mL}$ of THF in a dry-box under an argon atmosphere (ligands that could not be weighed in directly were added as $0.20 \mathrm{~mL}(0.0060 \mathrm{mmol})$ of a $0.030 \mathrm{M}$ stock solution in THF and no additional solvent was added). The vial was capped and stirred for $45 \mathrm{~min}$. Next, $19 \mathrm{mg}(0.11 \mathrm{mmol})$ of allylboronic acid pinacol ester was added followed by $0.31 \mathrm{~mL}(0.10 \mathrm{mmol})$ of a $0.325 \mathrm{M}$ stock solution of (1E, 4E)-1-phenyldeca-1,4-dien-3-one in THF. The vial was capped, taped with electrical tape, removed from the dry-box, and allowed to stir at ambient temperature for the time indicated in Table 1. After this time period, water was added and the mixture transferred to a separatory funnel with $\mathrm{CH}_{2} \mathrm{Cl}_{2}$. After gently swirling the layers, the organic layer was collected and the aqueous layer washed with $\mathrm{CH}_{2} \mathrm{Cl}_{2}(1 \mathrm{x})$. The combined organic layers were dried with $\mathrm{Na}_{2} \mathrm{SO}_{4}, a$ and volatiles were removed under reduced pressure. NMR analysis of the crude mixture was used to determine the regioselectivity of the reaction. Yields were determined after isolation of pure material, as a mixture of isomers, using silica gel chromatography (hexanes/EtOAc).

Procedure using $\mathrm{Ni}(\mathbf{c o d})_{2}$ : An oven-dried $20 \mathrm{~mL}$ scintillation vial equipped with a magnetic stir-bar was charged with $6.0 \mathrm{mg}$ (0.022 mmol) of bis(1,5-cyclooctadiene)nickel, $0.0438 \mathrm{mmol}$ of phosphine ligand, and $1.46 \mathrm{~mL}$ of THF in a dry-box under an argon atmosphere. The vial was capped and stirred for $45 \mathrm{~min}$. Next, $44.2 \mathrm{mg}(0.263 \mathrm{mmol})$ of allylboronic acid pinacol ester was added followed by $50.0 \mathrm{mg}(0.219 \mathrm{mmol})$ of $(1 E$, $4 E$ )-1-phenyldeca-1,4-dien-3-one. The vial was capped, taped with electrical tape, removed from the dry-box, and allowed to stir at ambient temperature for $4 \mathrm{~h}$. After this time period, $15 \mathrm{~mL}$ of water was added and the mixture transferred to a separatory funnel with $\mathrm{CH}_{2} \mathrm{Cl}_{2}$. After gently swirling the layers, the organic layer was collected and the aqueous layer washed with $\mathrm{CH}_{2} \mathrm{Cl}_{2}(1 \mathrm{x})$. The combined organic layers were dried with $\mathrm{Na}_{2} \mathrm{SO}_{4}$, and volatiles were removed under reduced pressure. NMR analysis of the crude mixture was used to determine the regioselectivity of the reaction. Yields were determined after isolation of pure material, as a mixture of isomers, using silica gel chromatography (hexanes/EtOAc).

\section{Procedure for the Ni-catalyzed conjugate allylation of styryl enones (Table 2).}

The conjugate allylation of styryl-activated enones using $\mathrm{Ni}(\mathrm{cod})_{2}$ was performed using the same procedure described above for the reaction in Table 1 using $\mathrm{Ni}(\mathrm{cod})_{2}$, where tricyclohexylphosphine was the ligand used. Reactions were performed using $50.0 \mathrm{mg}$ of substrate and run at the temperature and for the time indicated in Table 2. For the allylation reaction using (E)-5-methyl-1-phenylhexa-1,4-dien-3-one (entry 8) as the substrate, 2.0 equiv of allylboronic acid pinacol ester was used. For substrates bearing TBS-protected alcohols (entry 5, 6), buffer $(\mathrm{pH}=7)$ was used instead of $\mathrm{H}_{2} \mathrm{O}$ in the extraction step. The reaction utilizing $(1 E$, $4 E$ )-4-methyl-1-phenylhexa-1,4-dien-3-one as the substrate (entry 9) was quenched with 2.2 equiv of $1.0 \mathrm{M}$ $\mathrm{HCl}$ in $\mathrm{Et}_{2} \mathrm{O}$ under $\mathrm{N}_{2}$ before adding $\mathrm{H}_{2} \mathrm{O}$. 


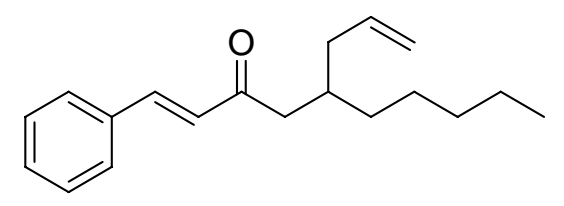

E)-5-allyl-1-phenyldec-1-en-3-one. An oil. Regioisomers were separable using iterative column chromatography $\left(\mathrm{SiO}_{2}\right.$ /hexanes:EtOAc). $\mathrm{R}_{f}$ (major) $=$ 0.22 (18:1 Hexanes:EtOAc); $\mathrm{R}_{f}$ (minor) $=0.19$ (18:1 Hexanes:EtOAc); IR (major, neat): 3064 (w), 2948 (s), 2917 (s), 2858 (s), 1942 (w), 1821 (w), 1689 (s), 1654 (s), 1608 (s), 1445 (s), 1320 (m), 1167 (m) cm ${ }^{-1} ;{ }^{1} \mathrm{H}$ NMR: $\delta$ (major) 7.48-7.59 (3H, m), 7.37 $(3 \mathrm{H}, \mathrm{m}), 6.72(1 \mathrm{H}, \mathrm{d}, J=16 \mathrm{~Hz}), 5.76(1 \mathrm{H}, \mathrm{m}), 5.01(2 \mathrm{H}, \mathrm{d}, J=12 \mathrm{~Hz}), 2.61(1 \mathrm{H}, \mathrm{dd}, J=16 \mathrm{~Hz}, J=6.0 \mathrm{~Hz})$, 2.52 (1H, dd, $J=16 \mathrm{~Hz}, J=6.0 \mathrm{~Hz}$ ), 1.95-2.20 (3H, m), 1.15-1.40 (8H, m), 0.86 (3H, t, $J=7.2 \mathrm{~Hz}) ; \delta$ (minor) 7.20-7.30 (2H, m), 7.10-7.20 (3H, m), $6.72(1 \mathrm{H}, \mathrm{dt}, J=16 \mathrm{~Hz}, J=7.2 \mathrm{~Hz}), 5.99(1 \mathrm{H}, \mathrm{d}, J=16 \mathrm{~Hz}), 5.63(1 \mathrm{H}$, m), $4.94(2 \mathrm{H}, \mathrm{m}), 3.29$ (1H, p, $J=7.2 \mathrm{~Hz}), 2.82(2 \mathrm{H}, \mathrm{m}), 2.37$ (2H, t, $J=7.2 \mathrm{~Hz}), 2.13$ (2H, q, $J=6.8 \mathrm{~Hz}), 1.38$ (2H, p, $J=7.2 \mathrm{~Hz}$ ), $1.17-1.32(6 \mathrm{H}, \mathrm{m}), 0.87$ (3H, t, $J=6.8 \mathrm{~Hz}$ ); ${ }^{13} \mathrm{C}$ NMR: $\delta$ (major) 200.4, 142.2, 136.6, 134.6, 130.3, 128.9, 128.2, 126.6, 116.6, 45.23, 38.26, 34.07, 33.80, 31.97, 26.40, 22.58, 14.03. LRMS (ESI+) Calc’d for $\mathrm{C}_{19} \mathrm{H}_{26} \mathrm{O}(\mathrm{M}+\mathrm{Na})^{+}$: 293.2 Found $(\mathrm{M}+\mathrm{Na})^{+}$: 293.2.

${ }^{1} \mathrm{H}$ NMR analysis of Crude Reaction Mixture (400 MHz, $\left.C D C l_{3}\right)$ :

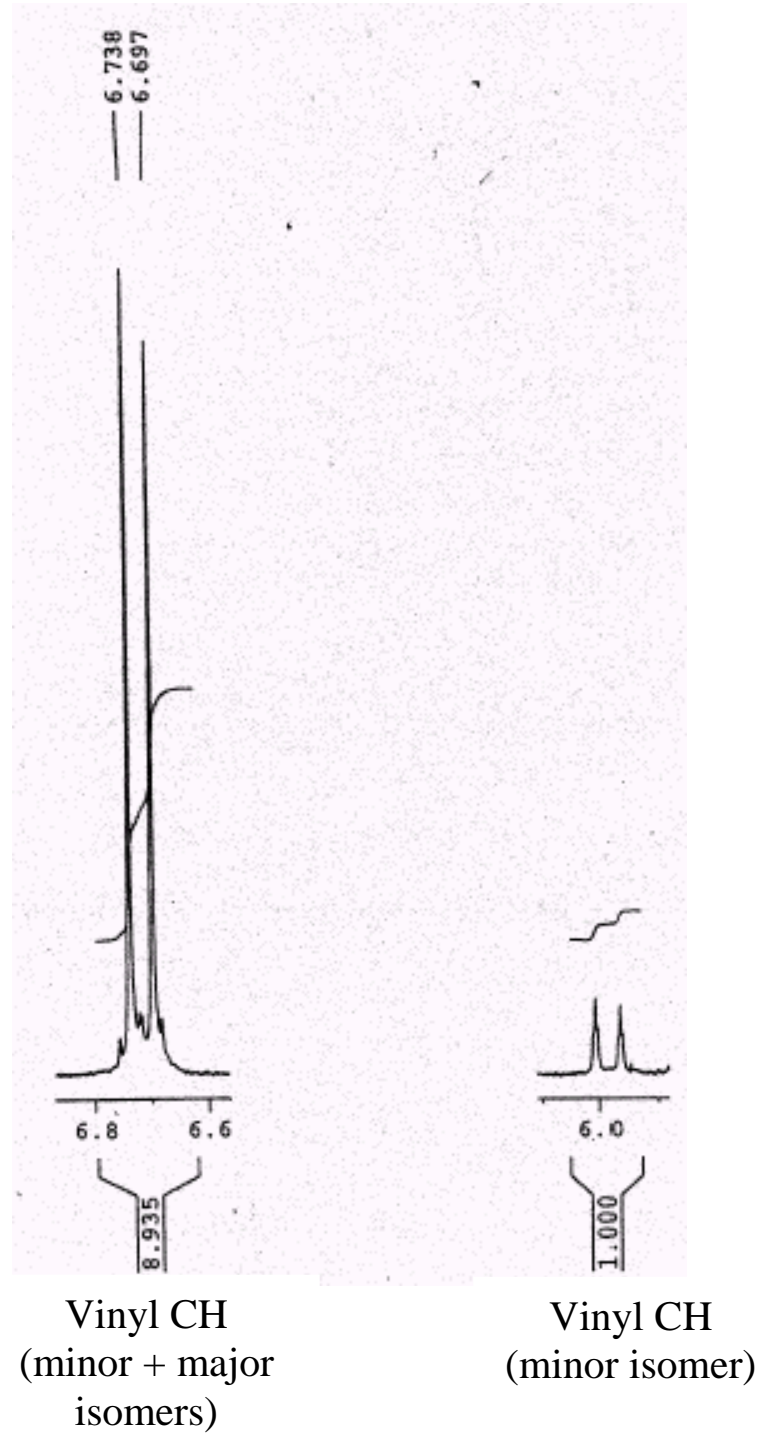




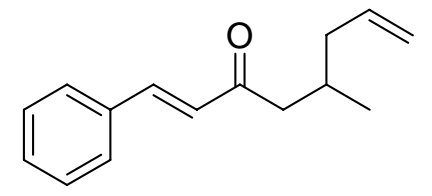

(E)-5-methyl-1-phenylocta-1,7-dien-3-one. $\quad$ An oil. $\quad \mathrm{R}_{f}=0.24 \quad(12: 1$ Hexanes:EtOAc); IR (neat): 3064 (m), 2955 (s), 2917 (s), 1946 (w), 1829 (w), 1689 (s), 1666 (s), 1611 (s), 1452 (s), 1324 (s), 1173 (s) cm ${ }^{-1}$; ${ }^{1} \mathrm{H}$ NMR: $\delta$ 7.45-7.57 (3H, m), 7.28-7.44 (3H, m), $6.72(1 \mathrm{H}, \mathrm{d}, J=16 \mathrm{~Hz}), 5.77(1 \mathrm{H}, \mathrm{m}), 5.02(2 \mathrm{H}, \mathrm{d}, J=14 \mathrm{~Hz})$, 2.66 (1H, dd, $J=16 \mathrm{~Hz}, J=6.0 \mathrm{~Hz}), 2.43(1 \mathrm{H}, \mathrm{dd}, J=16 \mathrm{~Hz}, J=8.0 \mathrm{~Hz}), 2.19(1 \mathrm{H}, \mathrm{m}), 1.95-2.12(2 \mathrm{H}, \mathrm{m})$, 0.95 (3H, d, $J=6.4 \mathrm{~Hz}$ ); ${ }^{13} \mathrm{C}$ NMR: $\delta 200.2,142.4,136.7,134.5,130.4,128.9,128.2,126.5,116.5,47.43$, 41.20, 29.52, 19.77. LRMS (ESI+) Calc'd for $\mathrm{C}_{15} \mathrm{H}_{18} \mathrm{O}(\mathrm{M}+\mathrm{Na})^{+}: 237.1$ Found $(\mathrm{M}+\mathrm{Na})^{+}:$237.1.

${ }^{1} \mathrm{H} N \mathrm{NR}$ analysis of Crude Reaction Mixture $\left(400 \mathrm{MHz}, \mathrm{CDCl}_{3}\right)$ :

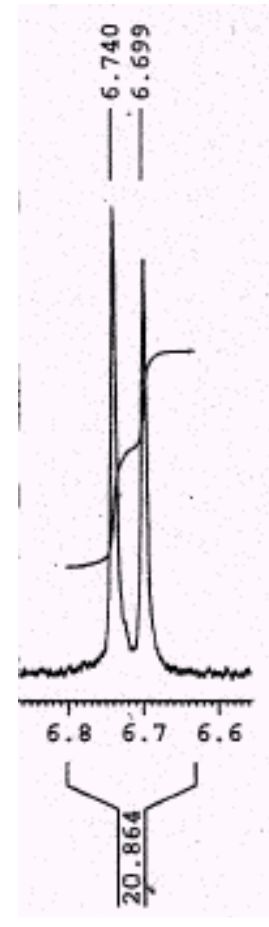

Vinyl CH

(minor + major isomers)

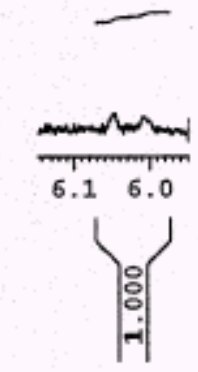

Vinyl CH

(minor isomer) 


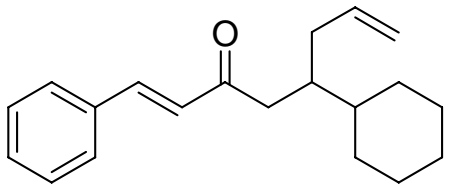

(E)-5-cyclohexyl-1-phenylocta-1,7-dien-3-one. An oil. Regioisomers were inseparable using column chromatography $\left(\mathrm{SiO}_{2}\right.$ /hexanes:EtOAc). $\mathrm{R}_{f}=0.29(12: 1$ Hexanes:EtOAc); IR (neat): 3056 (m), 2913 (s), 2842 (s), 1953 (w), 1817 (w), 1685 (s), 1662 (s), 1603 (s), 1441 (s), 1320 (m), 1181 (m) cm ${ }^{-1}$; ${ }^{1} \mathrm{H}$ NMR: $\delta 7.52$ (3H, m, major), 7.37 (3H, m, major), 7.25 (2H, m, minor), 7.16 (3H, m, minor), 6.72 (1H, d, $J=16 \mathrm{~Hz}$, major), $6.65(1 \mathrm{H}, \mathrm{dd}, J=16 \mathrm{~Hz}, J=6.8 \mathrm{~Hz}$, minor), $5.93(1 \mathrm{H}, \mathrm{d}, J=16 \mathrm{~Hz}, \operatorname{minor}), 5.74(1 \mathrm{H}, \mathrm{m}$, major), $5.63(1 \mathrm{H}, \mathrm{m}$, minor), 4.90-5.05 (4H, m, major \& minor), $3.28(1 \mathrm{H}, \mathrm{p}, J=7.2 \mathrm{~Hz}$, minor), 2.81 (2H, m, minor), 2.61 (1H, dd, $J$ $=16 \mathrm{~Hz}, J=6.0 \mathrm{~Hz}$, major) $2.52(1 \mathrm{H}, \mathrm{dd}, J=16 \mathrm{~Hz}, J=6.8 \mathrm{~Hz}$, major), 2.37 ( $2 \mathrm{H}, \mathrm{t}, J=7.2 \mathrm{~Hz}$, minor), 2.16 (1H, m, major), 1.91-2.10 (3H, m, major \& minor), 1.57-1.78 (10H, m, major \& minor), $1.36(1 \mathrm{H}, \mathrm{m}$, major), 0.94-1.29 (10H, m, major \& minor); ${ }^{13} \mathrm{C}$ NMR: $\delta$ 200.4, 199.5, 152.3, 144.2, 142.0, 137.4, 136.2, 134.6, 130.2, 128.8, 128.3, 128.2, 128.0, 127.5, 126.5, 126.2, 116.6, 116.2, 46.10, 42.55, 41.05, 40.59, 40.54, 40.40, 39.20, 35.90, 31.73, 30.06, 29.68, 26.71, 25.90, 25.68. LRMS (ESI+) Calc'd for $\mathrm{C}_{20} \mathrm{H}_{26} \mathrm{O}(\mathrm{M}+\mathrm{Na})^{+}: 305.2$ Found (M $+\mathrm{Na})^{+}: 305.2$.

${ }^{1} \mathrm{H} N \mathrm{NR}$ analysis of Crude Reaction Mixture (400 MHz, $\left.\mathrm{CDCl}_{3}\right)$ :

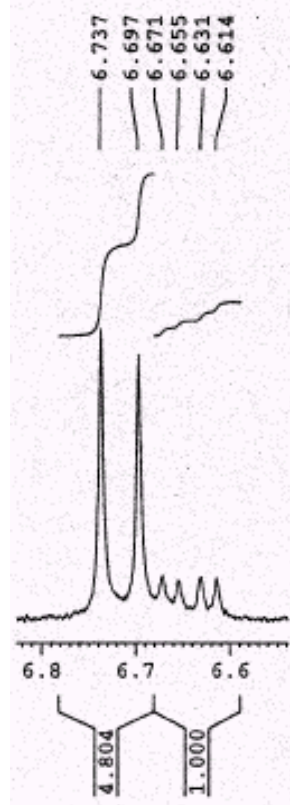

Vinyl CH

(minor + major isomers) 


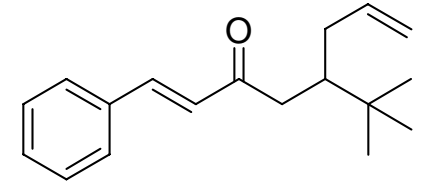

(E)-5-t-butyl-1-phenylocta-1,7-dien-3-one. $\quad$ An oil. $\quad \mathrm{R}_{f}=0.24 \quad(15: 1$ Hexanes:EtOAc); IR (neat): 3064 (m), 2959 (s), 2870 (m), 1953 (w), 1814 (w), 1689 (s), $1654(\mathrm{~s}), 1608$ (s), 1445 (s), 1363 (s), 1185 (m) cm ${ }^{-1}$; ${ }^{1} \mathrm{H}$ NMR: $\delta$ 7.48-7.57 (3H, m), $7.37(3 \mathrm{H}, \mathrm{m}), 6.73(1 \mathrm{H}, \mathrm{d}, J=16 \mathrm{~Hz}), 5.72(1 \mathrm{H}, \mathrm{m}), 4.97(1 \mathrm{H}, \mathrm{d}, J=17 \mathrm{~Hz}), 4.91$ $(1 \mathrm{H}, \mathrm{d}, J=10 \mathrm{~Hz}) 2.66(1 \mathrm{H}, \mathrm{dd}, J=17 \mathrm{~Hz}, J=4.0 \mathrm{~Hz}), 2.46(1 \mathrm{H}, \mathrm{dd}, J=17 \mathrm{~Hz}, J=6.0 \mathrm{~Hz}), 2.32-2.41(1 \mathrm{H}$, m), $2.13(1 \mathrm{H}, \mathrm{m}), 1.78(1 \mathrm{H}, \mathrm{m}), 0.89(9 \mathrm{H}, \mathrm{s}) ;{ }^{13} \mathrm{C}$ NMR: $\delta$ 200.1, 141.6, 138.4, 134.5, 130.1, 128.7, 128.0, 126.3, 115.8, 42.47, 41.99, 35.65, 33.28, 27.50. LRMS (ESI+) Calc'd for $\mathrm{C}_{18} \mathrm{H}_{24} \mathrm{O}(\mathrm{M}+\mathrm{Na})^{+}:$279.2 Found (M $+\mathrm{Na})^{+}: 279.2$.

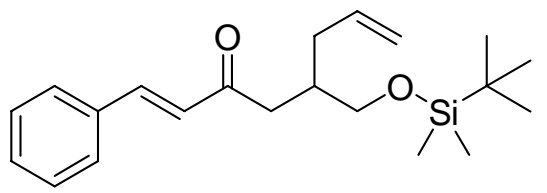

(E)-5-(t-butyldimethylsilyloxy)methyl)-1-phenylocta-1,7-dien-3-one. An oil. Regioisomers were separable using column chromatography ( $\mathrm{SiO}_{2}$ /hexanes:EtOAc). $\mathrm{R}_{f}=0.26$ (14:1 Hexanes:EtOAc); IR (neat): 3076 (m), 2955 (s), 2924 (s), 2854 (s), 1689 (s), 1662 (s), 1611 (s), 1471 (m), 1363 (m), 1251 (s), 1103 (s) $\mathrm{cm}^{-1} ;{ }^{1} \mathrm{H}$ NMR: $\delta$ 7.49-7.59 (3H, m), 7.37 (3H, m), $6.72(1 \mathrm{H}, \mathrm{d}, J=16 \mathrm{~Hz}), 5.77(1 \mathrm{H}, \mathrm{m}), 5.03(2 \mathrm{H}, \mathrm{m}), 3.56(1 \mathrm{H}, \mathrm{dd}, J=10 \mathrm{~Hz}, J=4.8 \mathrm{~Hz}), 3.51(1 \mathrm{H}, \mathrm{dd}, J=10$ $\mathrm{Hz}, J=5.6 \mathrm{~Hz}), 2.77(1 \mathrm{H}, \mathrm{dd}, J=16 \mathrm{~Hz}, J=6.8 \mathrm{~Hz}), 2.53(1 \mathrm{H}, \mathrm{dd}, J=16 \mathrm{~Hz}, J=6.0 \mathrm{~Hz}), 2.11-2.31(2 \mathrm{H}, \mathrm{m})$, 2.07 (1H, m), 0.87 (9H. s), 0.015 (3H, s), 0.0030 (3H, s); ${ }^{13} \mathrm{C}$ NMR: $\delta 200.2,142.3,136.5,134.6,130.3,128.9$, 128.2, 126.7, 116.6, 64.73, 41.91, 36.94, 35.63, 25.89, 18.26, -5.46. LRMS (ESI+) Calc'd for $\mathrm{C}_{21} \mathrm{H}_{32} \mathrm{O}_{2} \mathrm{Si}(\mathrm{M}+$ $\mathrm{Na})^{+}:$367.2 Found $(\mathrm{M}+\mathrm{Na})^{+}: 367.3$.

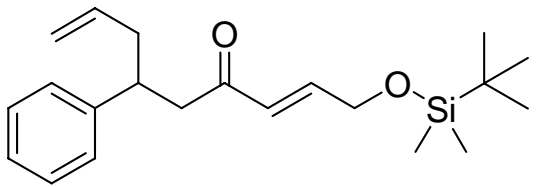

(E)-1-(t-butyldimethylsilyloxy)-6-phenylnona-2,8-dien-4-one. An oil. Regioisomers were separable using column chromatography $\left(\mathrm{SiO}_{2} /\right.$ hexanes:EtOAc). $\mathrm{R}_{f}=0.18$ (14:1 Hexanes:EtOAc); IR (neat): $3056(\mathrm{~m})$, 3029 (m), 2948 (s), 2924 (s), 2858 (s), 1697 (s), 1670 (s), 1635 (s), 1468 (m), $1359(\mathrm{~m}), 1251$ (s), 1134 (s) cm ${ }^{-1}$; ${ }^{1} \mathrm{H}$ NMR: $\delta$ 7.20-7.30 (2H, m), 7.10-7.20 (3H, m), $6.76(1 \mathrm{H}, \mathrm{dt}, J=16 \mathrm{~Hz}, J$ $=3.2 \mathrm{~Hz}), 6.28(1 \mathrm{H}, \mathrm{d}, J=16 \mathrm{~Hz}), 5.63(1 \mathrm{H}, \mathrm{m}), 4.96(1 \mathrm{H}, \mathrm{d}, J=16 \mathrm{~Hz}), 4.93(1 \mathrm{H}, \mathrm{d}, J=10 \mathrm{~Hz}), 4.28(2 \mathrm{H}, \mathrm{m})$, $3.31(1 \mathrm{H}, \mathrm{p}, J=7.2 \mathrm{~Hz}), 2.85(2 \mathrm{H}, \mathrm{m}), 2.37(2 \mathrm{H}, \mathrm{t}, J=7.2 \mathrm{~Hz}), 0.90(9 \mathrm{H}, \mathrm{s}), 0.045(6 \mathrm{H}, \mathrm{s}) ;{ }^{13} \mathrm{C}$ NMR: $\delta 198.9$, 145.3, 144.2, 136.2, 128.4, 127.9, 127.5, 126.3, 116.7, 62.23, 46.70, 40.81, 40.60, 25.84, 18.35, -5.45. LRMS $(\mathrm{ESI}+)$ Calc'd for $\mathrm{C}_{21} \mathrm{H}_{32} \mathrm{O}_{2} \mathrm{Si}(\mathrm{M}+\mathrm{Na})^{+}: 367.2$ Found $(\mathrm{M}+\mathrm{Na})^{+}: 367.3$.

${ }^{1} \mathrm{H} N \mathrm{NR}$ analysis of Crude Reaction Mixture (400 $\left.\mathrm{MHz}, \mathrm{CDCl}_{3}\right)$ :

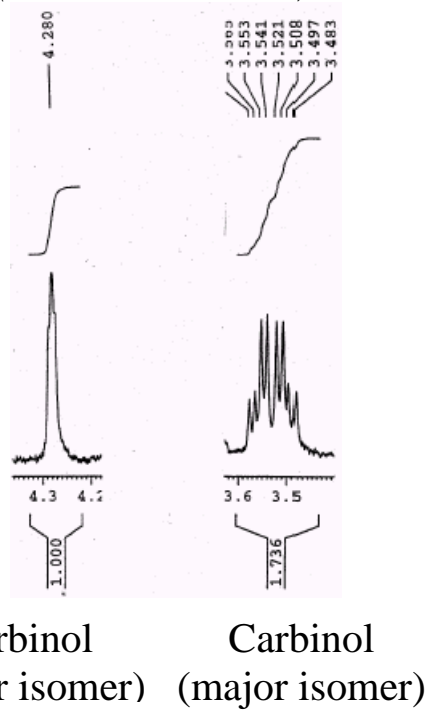




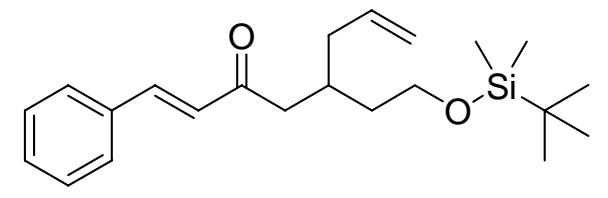

(E)-5-(2-t-butyldimethylsilyloxy)ethyl)-1-phenylocta-1,7-dien-3-one. An oil. Regioisomers were separable using iterative column chromatography ( $\mathrm{SiO}_{2} /$ hexanes:EtOAc). $\quad \mathrm{R}_{f} \quad$ (major) $=0.25$ (15:1 Hexanes:EtOAc); $\mathrm{R}_{f}$ (minor) = 0.18 (15:1 Hexanes:EtOAc); IR (major, neat): 3068 (m), 2948 (s), 2932 (s), 2847 (s), 1953 (w), 1817 (w), 1682 (s), 1658 (s), 1611 (s), 1468 (m), 1328 (m), 1254 (s) cm ${ }^{-1}$; ${ }^{1} \mathrm{H}$ NMR: $\delta$ (major) 7.45-7.57 (3H, m), 7.37 (3H, m), $6.72(1 \mathrm{H}, \mathrm{d}, J=16 \mathrm{~Hz}), 5.76(1 \mathrm{H}, \mathrm{m}), 5.02(2 \mathrm{H}, \mathrm{m}), 3.66(2 \mathrm{H}, \mathrm{m}) 2.63(2 \mathrm{H}, \mathrm{d}, J=6.4 \mathrm{~Hz}), 2.27(1 \mathrm{H}$, heptet, $J=$ 6.4 Hz), 2.01-2.20 (2H, m), 1.45-1.67 (2H, m), $0.86(9 \mathrm{H}, \mathrm{s}), 0.020(6 \mathrm{H}, \mathrm{s}) ; \delta$ (minor) 7.21-7.30 (2H, m), 7.10$7.20(3 \mathrm{H}, \mathrm{m}), 6.74(1 \mathrm{H}, \mathrm{dt}, J=16 \mathrm{~Hz}, J=7.2 \mathrm{~Hz}), 6.04(1 \mathrm{H}, \mathrm{d}, J=16 \mathrm{~Hz}), 5.62(1 \mathrm{H}, \mathrm{m}), 4.96(1 \mathrm{H}, \mathrm{d}, J=15$ $\mathrm{Hz}), 4.93(1 \mathrm{H}, \mathrm{d}, J=10 \mathrm{~Hz}), 3.67(2 \mathrm{H}, \mathrm{t}, J=6.4 \mathrm{~Hz}), 3.30(1 \mathrm{H}, \mathrm{p}, J=7.2 \mathrm{~Hz}), 2.83(2 \mathrm{H}, \mathrm{m}), 2.35(4 \mathrm{H}, \mathrm{m}), 0.87$ (9H, s), 0.023 (6H, s); ${ }^{13} \mathrm{C}$ NMR: $\delta$ (major) 200.1, 142.3, 136.4, 134.6, 130.3, 128.9, 128.2, 126.5, 116.9, 61.20, 45.29, 38.42, 36.61, 31.23, 25.92, 18.26, -5.34. LRMS (ESI+) Calc'd for $\mathrm{C}_{22} \mathrm{H}_{34} \mathrm{O}_{2} \mathrm{Si}(\mathrm{M}+\mathrm{Na})^{+}: 381.2$ Found $(\mathrm{M}+\mathrm{Na})^{+}: 381.3$.

${ }^{1} \mathrm{H}$ NMR analysis of Crude Reaction Mixture $\left(400 \mathrm{MHz}, \mathrm{CDCl}_{3}\right)$ :

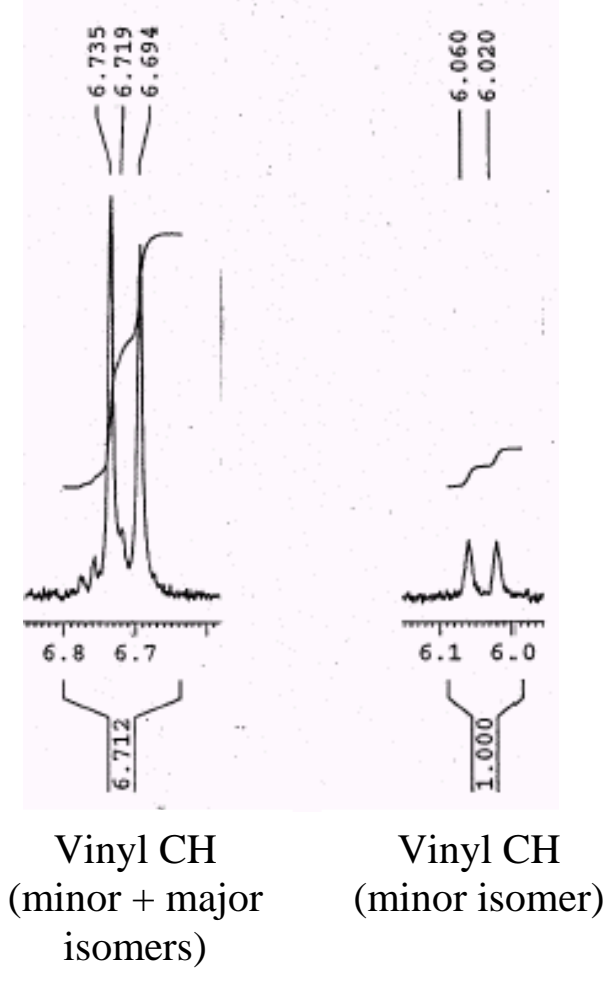




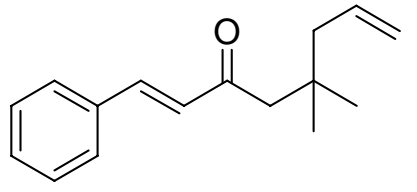

(E)-5,5-dimethyl-1-phenylocta-1,7-dien-3-one. An oil. $\mathrm{R}_{f}=0.26$ (14:1 Hexanes:EtOAc); IR (neat): 3072 (m), 2959 (s), 2862 (s), 1950 (w), 1814 (w), 1685 (s), 1651 (s), 1608 (s), 1440 (s), 1332 (s), 1200 (s) cm ${ }^{-1} ;{ }^{1} \mathrm{H}$ NMR: $\delta 7.52$ (2H, m), $7.49(1 \mathrm{H}, \mathrm{d}, J=16 \mathrm{~Hz}), 7.36$ (3H, m), $6.72(1 \mathrm{H}, \mathrm{d}, J=16 \mathrm{~Hz}), 5.84(1 \mathrm{H}, \mathrm{m}), 5.05$ (2H, m), $2.52(2 \mathrm{H}, \mathrm{s}), 2.12(2 \mathrm{H}, \mathrm{d}, J=7.6 \mathrm{~Hz}), 1.03(6 \mathrm{H}, \mathrm{s}) ;{ }^{13} \mathrm{C}$ NMR: $\delta 200.0,141.9,135.0,134.6,130.3$, 128.9, 128.3, 127.6, 117.7, 51.49, 46.86, 34.30, 27.40. LRMS (ESI+) Calc'd for $\mathrm{C}_{16} \mathrm{H}_{20} \mathrm{O}(\mathrm{M}+\mathrm{Na})^{+}: 251.1$ Found $(\mathrm{M}+\mathrm{Na})^{+}:$251.2.

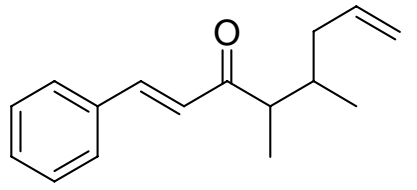

(E)-4,5-dimethyl-1-phenylocta-1,7-dien-3-one. An oil. Isolated as an inseparable mixture of diastereomers using column chromatography $\left(\mathrm{SiO}_{2}\right.$ /hexanes:EtOAc). $\mathrm{R}_{f}=$ 0.26 (14:1 Hexanes:EtOAc); IR (neat): 3076 (m), 2967 (s), 2920 (s), 1942 (w), 1821 (w), 1685 (s), 1651 (s), 1608 (s), 1449 (s), 1324 (s), 1188 (m) cm ${ }^{-1} ;{ }^{1} \mathrm{H}$ NMR: $\delta 7.58$ (2H, d, $J=16 \mathrm{~Hz}$, major \& minor), $7.54(4 \mathrm{H}, \mathrm{m}$, major \& minor), 7.37 (6H, major \& minor), 6.80 (1H, d, $J=16$ $\mathrm{Hz}$, major), $6.79(1 \mathrm{H}, \mathrm{d}, J=16 \mathrm{~Hz}$, minor), $5.76(2 \mathrm{H}, \mathrm{m}$, major \& minor), 5.02 (4H, m, major \& minor), 2.80 $(1 \mathrm{H}, \mathrm{p}, J=6.4 \mathrm{~Hz}$, minor), $2.73(1 \mathrm{H}, \mathrm{p}, J=6.8 \mathrm{~Hz}$, major), 1.85-2.28 (6H, m, major \& minor), 1.13 (3H, d, $J=$ $6.8 \mathrm{~Hz}$, major), 1.06 (3H, d, $J=7.2 \mathrm{~Hz}$, minor), 0.92 (3H, d, $J=6.4 \mathrm{~Hz}$, major), 0.84 (3H, d, $J=6.4 \mathrm{~Hz}$, minor); ${ }^{13}$ C NMR: $\delta$ 203.6, 203.5, 142.3, 136.9, 136.65, 136.59, 134.6, 130.4, 130.3, 128.9, 128.3, 128.27, 125.3, 125.1, 116.5, 116.4, 49.60, 48.47, 39.67, 37.42, 35.31, 34.59, 17.72, 15.25, 13.65, 11.35. LRMS (ESI+) Calc'd for $\mathrm{C}_{16} \mathrm{H}_{20} \mathrm{O}(\mathrm{M}+\mathrm{Na})^{+}:$251.1 Found $(\mathrm{M}+\mathrm{Na})^{+}: 251.1$.

\section{Procedure for the ring closing metathesis in Scheme 2.}

To $47.9 \mathrm{mg}(0.173 \mathrm{mmol})$ of 1,5-diphenyl-1,7-octadien-3-one was added $2.2 \mathrm{mg}(0.0035 \mathrm{mmol})$ of Hoveyda-Grubb's $2^{\text {nd }}$ generation catalyst (Garber, S. B.; Kingbury, J. S.; Gray, B. L.; Hoveyda, A. H. J. Am. Chem. Soc. 2000, 122, 8168) in a dry-box under Ar. This was diluted with $5.7 \mathrm{~mL}$ of dry, degassed $\mathrm{CH}_{2} \mathrm{Cl}_{2}$. A magnetic stir bar was added, followed by a septum, and the reaction was removed from the dry-box and stirred at room temperature under $\mathrm{N}_{2}$. After $1 \mathrm{~h}, 3$ drops (22 G needle) of t-butyl vinyl ether was added and volatiles removed under reduced pressure. Silica gel chromatography (pentane/ $\mathrm{Et}_{2} \mathrm{O}$ ) of the crude mixture afforded 28.1 mg (94\%) of 5-phenylcyclohex-2-enone whose spectral data was consistent with the literature (Rutherford, A. P.; Gibb, C. S.; Hartley, R. C.; Goodman, J. M. J. Chem. Soc. Perkin Trans. 1 2001, 1051.).

\section{Procedure for the Sn-catalyzed Baeyer-Villager oxidation in Scheme 2.}

For lead reference, see: Göttlich, R.; Yamakoshi, K.; Sasai, H.; Shibasaki, M. Synlett 1997, 971. In a 2dram vial with magnetic stir bar in a dry-box under Ar was weighed $\sim 30 \mathrm{mg}$ of crushed $4 \AA$ molecular sieves. Next, $33.8 \mu \mathrm{L}(0.0338 \mathrm{mmol})$ of a $1 \mathrm{M}$ solution of ( \pm )-trans-1,2-diaminocyclohexane in $\mathrm{CH}_{2} \mathrm{Cl}_{2}$ was added by syringe followed by dilution with $0.32 \mathrm{~mL}$ of $\mathrm{CH}_{2} \mathrm{Cl}_{2}$. Next, $33.8 \mu \mathrm{L}(0.0338 \mathrm{mmol})$ of a $1 \mathrm{M}$ solution of $\mathrm{SnCl}_{4}$ in $\mathrm{CH}_{2} \mathrm{Cl}_{2}$ was added and the vial was capped with a septum, removed from the dry-box and cooled to $0{ }^{\circ} \mathrm{C}$ (ice/brine). $\mathrm{TMS}_{2} \mathrm{O}_{2}$ was added dropwise as a $1 \mathrm{M}$ solution in $\mathrm{CH}_{2} \mathrm{Cl}_{2}(0.27 \mathrm{~mL}, 0.27 \mathrm{mmol})$. After stirring for $10 \mathrm{~min}$ at this temperature, $37.3 \mathrm{mg}(0.135 \mathrm{mmol})$ of 1,5-diphenyl-1,7-octadien-3-one was added in $0.59 \mathrm{~mL}$ $\mathrm{CH}_{2} \mathrm{Cl}_{2}$ via canula. The reaction became a blue-gray color and was subsequently warmed to room temperature and stirred for $15 \mathrm{~h}$. Sodium sulfite $(41 \mathrm{mg}$ ) was then added, and the reaction stirred for an additional $3 \mathrm{~h}$. Finally, the reaction was filtered through a pad of silica gel using EtOAc and concentrated under reduced pressure. Silica gel chromatography (hexanes/EtOAc) of the crude material afforded $31.5 \mathrm{mg}(80 \%)$ of (E)styryl-3-phenylhex-5-enoate as a white solid. 


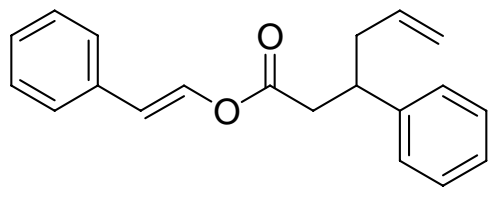

(E)-styryl-3-phenylhex-5-enoate. A white solid. $\mathrm{mp}=66-70^{\circ} \mathrm{C} . \quad \mathrm{R}_{f}=0.24$ (30:1 Hexanes:EtOAc); IR ( $\mathrm{CH}_{2} \mathrm{Cl}_{2}$ solution): $3087(\mathrm{~m}), 3024(\mathrm{~m}), 2917(\mathrm{~m})$, 1747 (s), 1652 (m), 1495 (m), 1212 (m), 1142 (s) cm ${ }^{-1}$; ${ }^{1} \mathrm{H}$ NMR: $\delta 7.74(1 \mathrm{H}, \mathrm{d}$, $J=13 \mathrm{~Hz}), 7.24-7.39$ (6H, m), 7.10-7.24 (4H, m), 6.03 (1H, d, $J=13 \mathrm{~Hz}), 5.66$ (1H, m), 5.02 (2H, m), $3.26(1 \mathrm{H}, \mathrm{p}, J=7.2 \mathrm{~Hz}), 2.81(1 \mathrm{H}, \mathrm{dd}, J=16 \mathrm{~Hz}, J=6.4 \mathrm{~Hz}), 2.68(1 \mathrm{H}, \mathrm{dd}, J=16 \mathrm{~Hz}, J$ = 8.4 Hz), $2.42(2 \mathrm{H}, \mathrm{m}) ;{ }^{13} \mathrm{C}$ NMR: $\delta$ 169.3, 143.1, 136.1, 135.6, 134.0, 128.6, 128.5, 127.3, 126.7, 126.1, 117.1, 115.2, 41.56, 40.61, 40.26. Note that the peak at 127.3 ppm was 2 overlapping signals that could not be resolved at higher frequency. ${ }^{13} \mathrm{C}$ analysis in $\mathrm{C}_{6} \mathrm{D}_{6}(125 \mathrm{MHz})$ resolved these peaks. This data $\left(\mathrm{C}_{6} \mathrm{D}_{6}\right.$ referenced at 128.39 relative to tetramethylsilane) was: $169.3,143.9,137.0,136.4,134.9,129.2,129.1,128.1$, 127.8, 127.3, 126.8, 117.4, 115.7, 42.22, 41.12, 40.63. LRMS (ESI+) Calc’d for $\mathrm{C}_{20} \mathrm{H}_{20} \mathrm{O}_{2}(\mathrm{M}+\mathrm{Na})^{+}: 315$ Found $(\mathrm{M}+\mathrm{Na})^{+}: 315$.

\section{Procedure for the hydrolysis of $(E)$-styryl-3-phenylhex-5-enoate (Scheme 2).}

To a solution of $26.3 \mathrm{mg}(0.900 \mathrm{mmol})$ of $(E)$-styryl-3-phenylhex-5-enoate in $0.68 \mathrm{~mL}$ of THF was added $0.22 \mathrm{~mL}$ of water, and the reaction then subsequently cooled to $0{ }^{\circ} \mathrm{C}$. $\mathrm{LiOH} \cdot \mathrm{H}_{2} \mathrm{O}(7.6 \mathrm{mg}, 0.18 \mathrm{mmol})$ was then added, and the reaction was stirred at this temperature and monitored by TLC. After complete consumption of the starting material (2-2.5 h), the reaction was acidified with $1 \mathrm{M} \mathrm{HCl}$ and extracted with EtOAc (3x). The organic layers were combined, washed with brine, and dried over anhydrous $\mathrm{Na}_{2} \mathrm{SO}_{4}$. Volatiles were removed under reduced pressure, and the product was purified using silica gel chromatography (1\% AcOH in $\mathrm{CH}_{2} \mathrm{Cl}_{2} / \mathrm{Et}_{2} \mathrm{O}, \mathrm{R}_{f}=0.25$ in $1 \% \mathrm{AcOH}$ in $20: 1 \mathrm{CH}_{2} \mathrm{Cl}_{2}: \mathrm{Et}_{2} \mathrm{O}$ ) to give $16.5 \mathrm{mg}$ (96\%) of 3-phenylhex-5-enoic acid after removal of $\mathrm{AcOH}$ by azeotropic distillation with toluene using a rotary evaporator followed by removal of toluene via azeotropic distillation with $\mathrm{CH}_{2} \mathrm{Cl}_{2}$. Spectral data was consistent with the literature (Allin, S. M.; Essat, M.; Pita, C. H.; Baird, R. D.; McKee, V.; Elsegood, M.; Edgar, M.; Andrews, D. M.; Shah, P.; Aspinall, I. Org. Biomol. Chem. 2005, 3, 809.).

\section{Procedure for the asymmetric conjugate allylation of dibenzylidene acetone (Scheme 4).}

An oven-dried 2-dram vial equipped with a magnetic stir-bar was charged with $2.5 \mathrm{mg}(0.0027 \mathrm{mmol})$ of tris(dibenzylideneacetone)dipalladium, $6.3 \mathrm{mg}(0.0064 \mathrm{mmol})$ of chiral ligand (for ligand synthesis see: Woodward, A. R.; Burks, H. E.; Chan, L. M.; Morken, J. P. Org. Lett. 2005, 7, 5505), and $0.71 \mathrm{~mL}$ of THF in a dry-box under an argon atmosphere. The vial was capped and stirred for $45 \mathrm{~min}$. Next, $19.8 \mathrm{mg}(0.118 \mathrm{mmol})$ of allylboronic acid pinacol ester was added followed by $25.0 \mathrm{mg}(0.107 \mathrm{mmol})$ of dibenzylidene acetone. The vial was capped, taped with electrical tape, removed from the dry-box, and allowed to stir at ambient temperature for $24 \mathrm{~h}$. After this time period, water was added and the mixture transferred to a separatory funnel with $\mathrm{CH}_{2} \mathrm{Cl}_{2}$. After gently swirling the layers, the organic layer was collected and the aqueous layer washed with $\mathrm{CH}_{2} \mathrm{Cl}_{2}(2 \mathrm{x})$. The combined organic layers were dried with $\mathrm{Na}_{2} \mathrm{SO}_{4}$, and volatiles were removed under reduced pressure. Silica gel chromatography (hexanes/EtOAc) afforded $26.3 \mathrm{mg}$ (83\%) of 1,5-diphenyl-1,7octadien-3-one whose spectral data has been reported previously (Mandal, S. K.; Amin, S. R.; Crowe, W. E. J. Am. Chem. Soc. 2001, 123, 6457.). The optical rotation was: $[\alpha]^{20}=+13^{\circ}\left(c=1.0, \mathrm{CHCl}_{3}\right)$. The absolute configuration was determined by performing the ring-closing metathesis shown in Scheme 2 on the chiral material and comparing the optical rotation $\left([\alpha]_{\mathrm{D}}^{20}=-42^{\circ}\left(c=0.5, \mathrm{CHCl}_{3}\right)\right)$ with the known value $(\mathrm{Hareau}, \mathrm{G}$. PJ.; Koiwa, M.; Hikichi, S; Sato, F. J. Am. Chem. Soc. 1999, 121, 3640). The enantiomeric excess was determined using chiral SFC (data shown below). 
Chiral SFC (AD-H, Chiralpak, $150 \mathrm{psi}, 50^{\circ} \mathrm{C}$, flow $\left.=3 \mathrm{~mL} / \mathrm{min}, 4 \% \mathrm{MeOH}\right)$ analysis of conjugate allylation product:

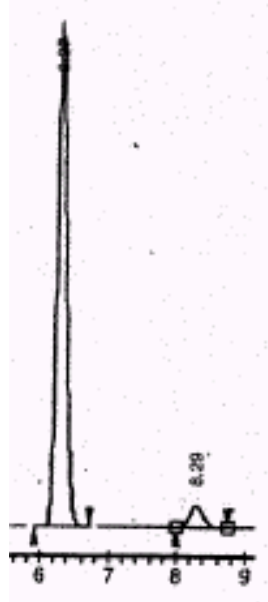

Allylation
product

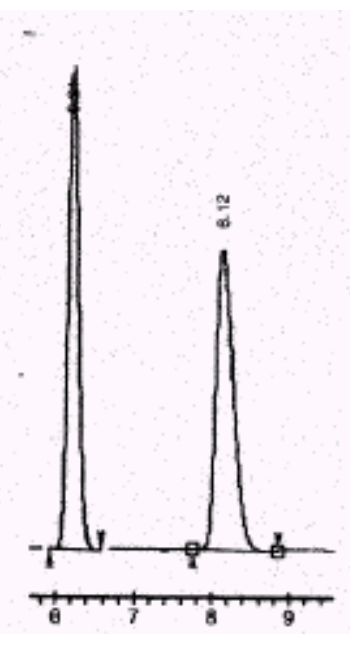

Racemic

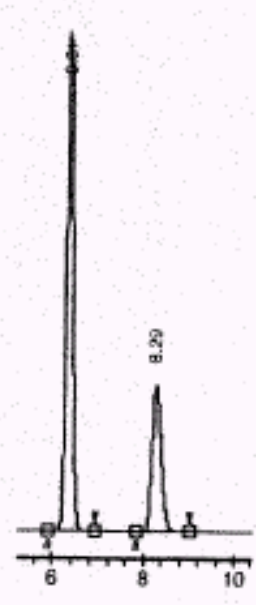

Allylation product + racemic coinjection

\begin{tabular}{|c|c|c|c|c|c|c|c|c|c|}
\hline$\#$ & Name & $\begin{array}{l}\text { Start } \\
\text { [Min] }\end{array}$ & $\begin{array}{l}\text { Time } \\
\text { [Min] }\end{array}$ & $\begin{array}{l}\text { End } \\
\text { [Min] }\end{array}$ & $\begin{array}{l}\text { RT } \\
\text { Oftset } \\
\text { [Min] }\end{array}$ & $\begin{array}{l}\text { Quantity } \\
{[\%} \\
\text { Area] }\end{array}$ & $\begin{array}{l}\text { Height } \\
{[\mu \mathrm{V}]}\end{array}$ & $\begin{array}{l}\text { Area } \\
\text { [uV.Min] }\end{array}$ & $\begin{array}{l}\text { Area } \\
{[\%]}\end{array}$ \\
\hline 1 & UNKNOWN & 5.91 & 6.27 & 6.72 & 0 & 94.41 & 24264.9 & 3924 & 94.412 \\
\hline 2 & UNKNOWN & 8 & 8.29 & 8.75 & 0 & 5.59 & 1032.6 & 232.3 & 5.588 \\
\hline Tanal & & & & -5 & & 100 & 2529975 & 41562 & 100 \\
\hline
\end{tabular}


Computational Methods: DFT calculations were performed using B3LYP ${ }^{1,2}$ with the basis set of Stuttgart RSC 1997 ECP $^{3}$ for Pd, $6-311+\mathrm{G}^{* 4}$ for others. All calculations were conducted using Gaussian 03 C02 package ${ }^{5}$ with tight SCF convergence and ultra fine integration grids on the 128-CPU SGI Altix 3700 SMP machine ${ }^{6}$ at University of North Carolina at Chapel Hill. In search for the transition state structure, a single-point frequency calculation has been performed to ensure that the final structure obtained (i) has only one imaginary frequency and (ii) the vibration mode of the negative frequency corresponds to the anticipated bond formation.

\section{Reactant $\left(3,3^{\prime}\right.$-Elmination $)$ :}
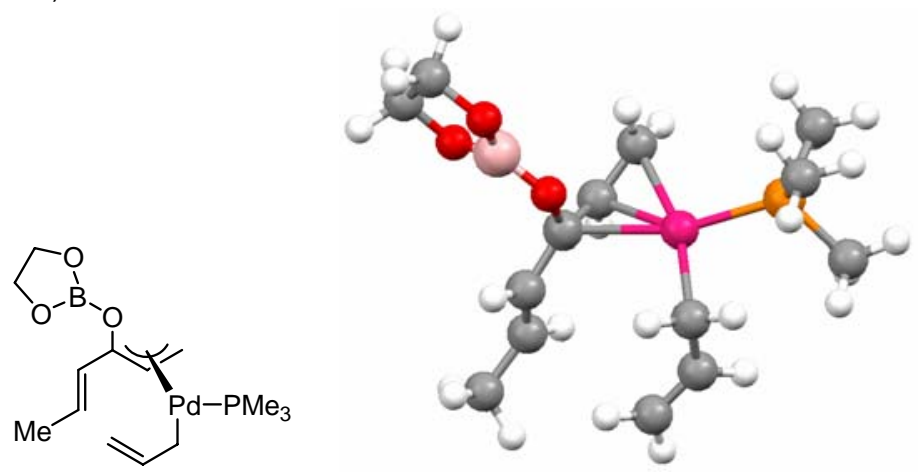

\begin{tabular}{|c|c|c|c|}
\hline C & -2.21400 & 4.32800 & 0.38200 \\
\hline $\mathrm{H}$ & -1.45700 & 5.10300 & ๑. 21800 \\
\hline $\mathrm{H}$ & -2.84100 & 4.67800 & 1.21100 \\
\hline $\mathrm{H}$ & -2.84300 & 4.27600 & -0.51100 \\
\hline B & -3.03100 & -1.03000 & -0.44800 \\
\hline $\mathrm{C}$ & -1.33600 & 0.53100 & ๑. 28900 \\
\hline $\mathrm{C}$ & 3.16900 & -2.19400 & -1.51400 \\
\hline C & 3.32900 & -2.44700 & 1.34000 \\
\hline $\mathrm{C}$ & 4.53300 & $-\odot .19900$ & 0.04200 \\
\hline C & -0.74800 & $\odot .10100$ & 1.49100 \\
\hline $\mathrm{C}$ & -5.12300 & -1.49800 & ๑. 29100 \\
\hline $\mathrm{C}$ & -4.73400 & -2.47100 & -0.84800 \\
\hline $\mathrm{C}$ & -1.84100 & 1.87100 & 0.02100 \\
\hline C & -0.18400 & -1.17200 & 1.69100 \\
\hline $\mathrm{C}$ & -1.58900 & 3.00300 & 0.69800 \\
\hline C & 1.25700 & 3.71300 & -0.75200 \\
\hline C & 1.57700 & 1.30800 & -1.42700 \\
\hline C & 1.96800 & 2.57100 & -0.79200 \\
\hline $\mathrm{H}$ & 3.09000 & -1.54300 & -2.38600 \\
\hline $\mathrm{H}$ & 4.13000 & -2.71600 & -1.54300 \\
\hline $\mathrm{H}$ & 2.36300 & -2.92800 & -1.57300 \\
\hline $\mathrm{H}$ & 2.52200 & -3.18100 & 1.36900 \\
\hline $\mathrm{H}$ & 4.27700 & -2.96500 & 1.16700 \\
\hline $\mathrm{H}$ & 3.36800 & -1.95300 & 2.31200 \\
\hline $\mathrm{H}$ & 4.60100 & 0.36200 & $\odot .97700$ \\
\hline $\mathrm{H}$ & 5.41800 & -0.83600 & -0.05300 \\
\hline $\mathrm{H}$ & 4.52400 & $\odot .51800$ & -0.78000 \\
\hline $\mathrm{H}$ & -5.44200 & -2.01000 & 1.20200 \\
\hline $\mathrm{H}$ & -5.90700 & -0.79700 & -0.00900 \\
\hline $\mathrm{H}$ & -4.59100 & -3.49500 & -0.48800 \\
\hline $\mathrm{H}$ & -5.45900 & -2.48500 & -1.66300 \\
\hline $\mathrm{H}$ & -2.49300 & 1.92600 & -0.85000 \\
\hline $\mathrm{H}$ & -0.91100 & 2.98700 & 1.54800 \\
\hline $\mathrm{H}$ & 2.38900 & ๑. 86900 & -2.01100 \\
\hline $\mathrm{H}$ & 0.69700 & 1.41000 & -2.06700 \\
\hline $\mathrm{H}$ & 2.93300 & 2.57100 & -0.28000 \\
\hline $\mathrm{H}$ & 0.24600 & -1.39700 & 2.66000 \\
\hline $\mathrm{H}$ & 1.62200 & 4.59000 & -0.22600 \\
\hline $\mathrm{H}$ & 0.30700 & 3.80900 & -1.26600 \\
\hline $\mathrm{H}$ & -0.57000 & $\odot .87000$ & 2.23700 \\
\hline $\mathrm{H}$ & -0.54600 & -2.02900 & 1.13300 \\
\hline 0 & -1.81600 & -0.44600 & -0.60200 \\
\hline 0 & -3.48000 & -1.97700 & -1.34000 \\
\hline 0 & -3.92400 & -0.75700 & 0.56300 \\
\hline $\mathrm{P}$ & 2.98200 & -1.19700 & $\odot .02600$ \\
\hline $\mathrm{Pd}$ & 1.00800 & -0.03800 & 0.15400 \\
\hline
\end{tabular}


Transition State $\left(3,3^{\prime}\right.$-Elmination):

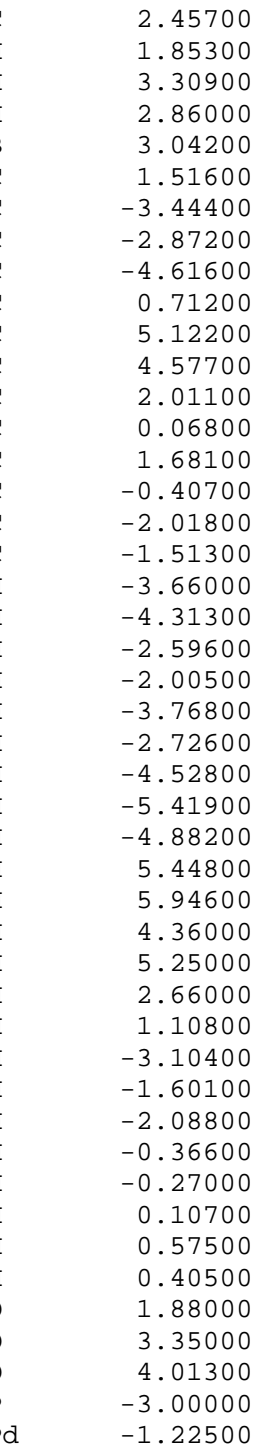
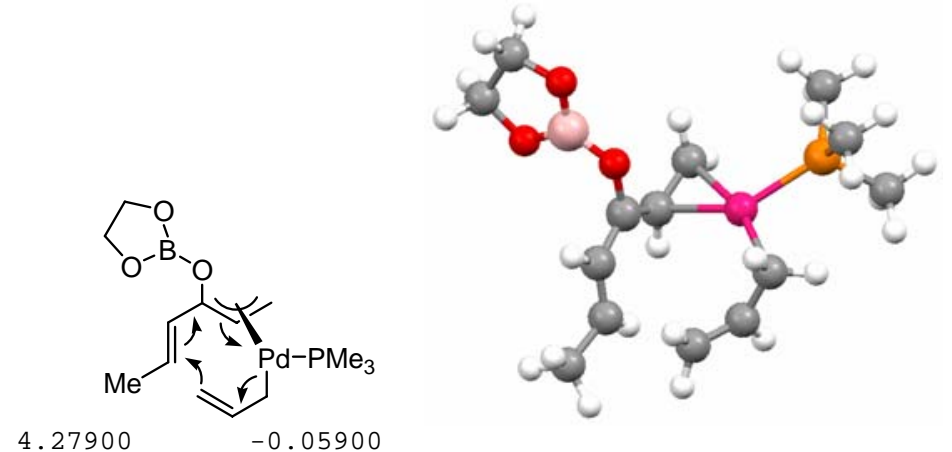
Reactant (1,2 addition to simple enone):

\begin{tabular}{|c|c|}
\hline C & 1.28100 \\
\hline C & $\odot .66100$ \\
\hline C & 0.11200 \\
\hline $\mathrm{H}$ & -0.39100 \\
\hline $\mathrm{H}$ & 0.52800 \\
\hline B & 3.14300 \\
\hline 0 & 3.68300 \\
\hline 0 & 4.01400 \\
\hline $\mathrm{Pd}$ & $-\odot .9700 \odot$ \\
\hline P & -2.97800 \\
\hline C & -3.19000 \\
\hline $\mathrm{H}$ & -3.07700 \\
\hline H & -4.17200 \\
\hline $\mathrm{H}$ & -2.41600 \\
\hline C & -3.38400 \\
\hline $\mathrm{H}$ & -2.61500 \\
\hline H & -4.35600 \\
\hline $\mathrm{H}$ & -3.39700 \\
\hline C & -4.48700 \\
\hline $\mathrm{H}$ & -4.55700 \\
\hline $\mathrm{H}$ & -5.39400 \\
\hline $\mathrm{H}$ & -4.42000 \\
\hline c & 5.28300 \\
\hline $\mathrm{H}$ & 5.66800 \\
\hline $\mathrm{H}$ & 5.98200 \\
\hline C & 4.98400 \\
\hline $\mathrm{H}$ & 4.95100 \\
\hline $\mathrm{H}$ & 5.70000 \\
\hline C & -1.56200 \\
\hline $\mathrm{H}$ & -2.35600 \\
\hline H & -1.99700 \\
\hline c & -0.47800 \\
\hline $\mathrm{H}$ & -0.10300 \\
\hline C & 0.09500 \\
\hline $\mathrm{H}$ & -0.23400 \\
\hline $\mathrm{H}$ & 0.89800 \\
\hline 0 & 1.87000 \\
\hline H & $\odot .40600$ \\
\hline C & 1.78800 \\
\hline H & 2.86800 \\
\hline $\mathrm{H}$ & 1.29300 \\
\hline H & 1.59906 \\
\hline
\end{tabular}

\begin{tabular}{|c|c|}
\hline$\odot .44700$ & -0.93400 \\
\hline$-\odot .55700$ & -1.69600 \\
\hline-1.73500 & -1.15900 \\
\hline-2.42200 & -1.82900 \\
\hline-2.17900 & $-\odot .2600 \odot$ \\
\hline-0.35600 & $\odot .40500$ \\
\hline-0.66400 & 1.63300 \\
\hline-0.55800 & -0.64400 \\
\hline$\odot .0790 \odot$ & -0.36400 \\
\hline$-\odot .82600$ & $\odot .3090 \odot$ \\
\hline-0.93900 & 2.13900 \\
\hline 0.05100 & 2.58400 \\
\hline-1.34100 & 2.40600 \\
\hline-1.58400 & 2.55700 \\
\hline-2.54600 & $-\odot .2270 \odot$ \\
\hline-3.23200 & 0.13100 \\
\hline$-2.8700 \odot$ & $\odot .15600$ \\
\hline-2.59600 & -1.31700 \\
\hline$\odot .0970 \odot$ & -0.21400 \\
\hline$\odot .09600$ & -1.30300 \\
\hline-0.35300 & 0.20000 \\
\hline 1.13400 & $\odot .11800$ \\
\hline-0.93200 & $-\odot .08600$ \\
\hline-1.80200 & -0.62200 \\
\hline-0.10100 & -0.21700 \\
\hline-1.22100 & 1.40500 \\
\hline-2.29300 & 1.62100 \\
\hline$-\odot .7500 \odot$ & 2.08200 \\
\hline 2. 01900 & $\odot .38300$ \\
\hline 1.84600 & 1.11800 \\
\hline 2.55300 & -0.47000 \\
\hline 2.78800 & 1.01500 \\
\hline 2.38900 & 1.95900 \\
\hline 3.91100 & 0.55200 \\
\hline 4.38100 & -0.37200 \\
\hline 4.40500 & 1.09000 \\
\hline ๑.0950९ & 0.29200 \\
\hline-0.28300 & -2.71600 \\
\hline 1.72400 & -1.52100 \\
\hline 1.66700 & -1.70000 \\
\hline 1.93700 & -2.47100 \\
\hline 2.56200 & -0.8430 \\
\hline
\end{tabular}


Transition State (1,2 addition to simple enone):

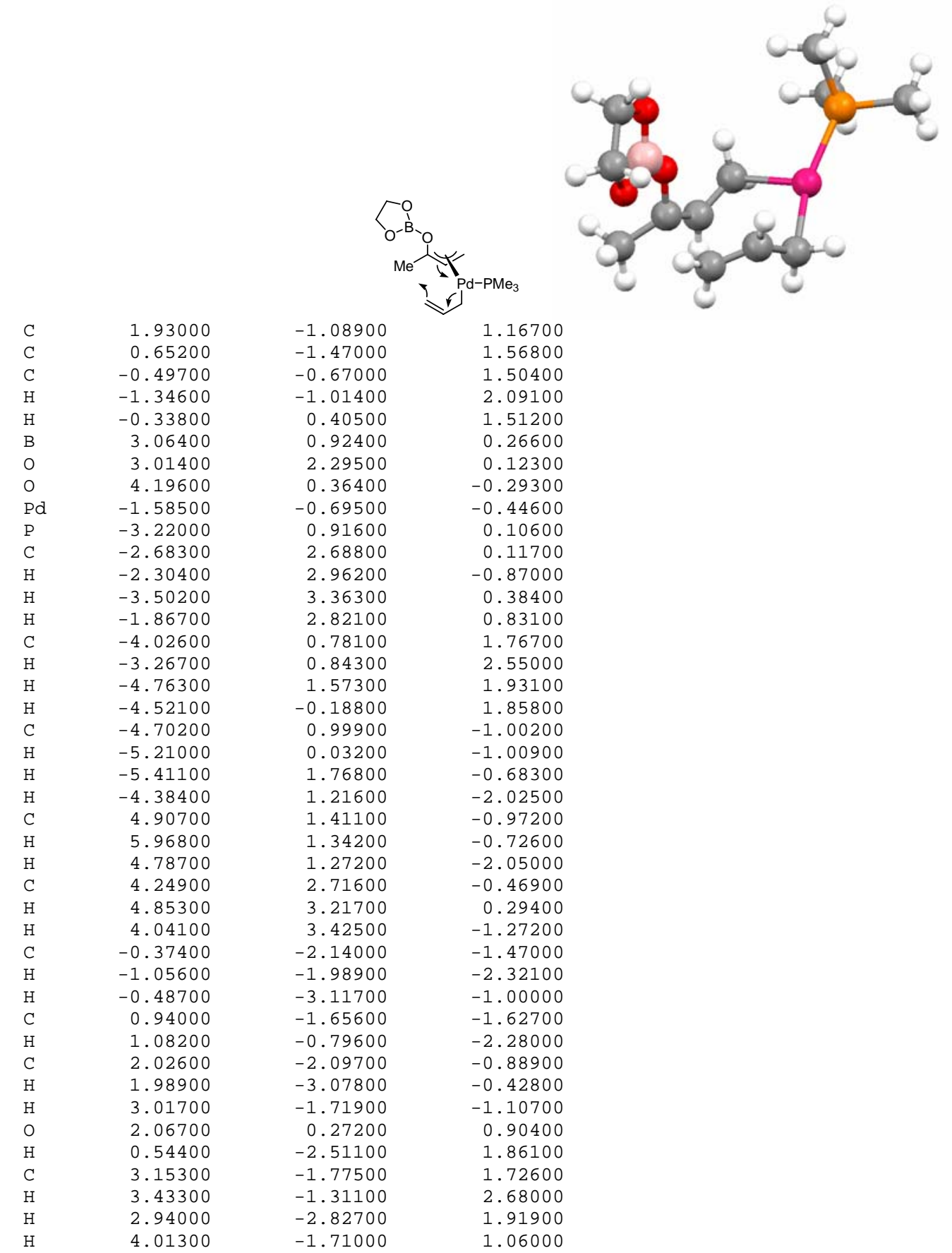


Reactant (1,4 addition to simple enone):

\begin{tabular}{|c|c|}
\hline C & 1.24400 \\
\hline C & 0.77800 \\
\hline C & $\odot .48700$ \\
\hline H & $\odot .07900$ \\
\hline $\mathrm{H}$ & 1.07400 \\
\hline B & 3.22600 \\
\hline 0 & 3.85300 \\
\hline 0 & 4.05300 \\
\hline $\mathrm{Pd}$ & -0.92400 \\
\hline $\mathrm{P}$ & -2.15200 \\
\hline C & -3.95100 \\
\hline $\mathrm{H}$ & -4.41800 \\
\hline $\mathrm{H}$ & -4.46400 \\
\hline H & -4.08100 \\
\hline C & -1.64900 \\
\hline $\mathrm{H}$ & -1.70200 \\
\hline H & -2.28800 \\
\hline $\mathrm{H}$ & -0.61600 \\
\hline C & -2.17500 \\
\hline H & -1.15700 \\
\hline H & -2.79200 \\
\hline H & -2.56300 \\
\hline C & 5.37600 \\
\hline H & 5.80000 \\
\hline H & 5.99800 \\
\hline C & 5.18300 \\
\hline $\mathrm{H}$ & 5.24700 \\
\hline $\mathrm{H}$ & 5.89200 \\
\hline C & -2.29100 \\
\hline $\mathrm{H}$ & -2.89900 \\
\hline H & -1.69800 \\
\hline C & -3.12800 \\
\hline $\mathrm{H}$ & -3.89900 \\
\hline C & -3.03500 \\
\hline $\mathrm{H}$ & -2.30000 \\
\hline $\mathrm{H}$ & -3.68800 \\
\hline 0 & 1.91400 \\
\hline $\mathrm{H}$ & 0.40700 \\
\hline C & 1.44000 \\
\hline $\mathrm{H}$ & 2.48700 \\
\hline $\mathrm{H}$ & $\odot .81400$ \\
\hline $\mathrm{H}$ & 1.19600 \\
\hline
\end{tabular}
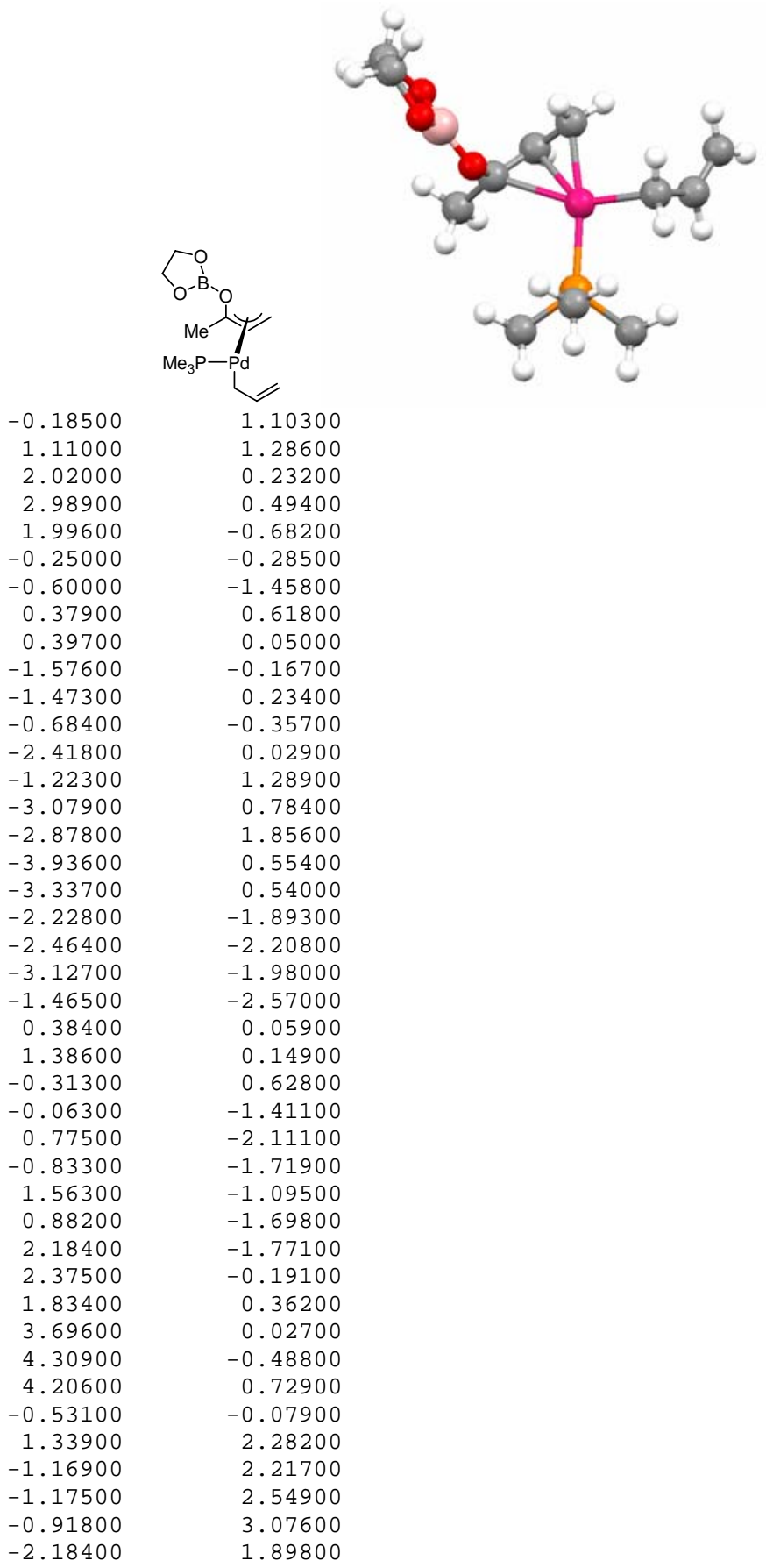

1.10300

1.28600

0.23200

0.49400

$-0.68200$

$-\odot .28500$

$-1.45800$

0.61800

0.05000

$-0.16700$

$\odot .23400$

$-0.35700$

0.02900

1.28900

0.78400

1.85600

0.55400

0.54000

$-1.89300$

$-2.20800$

$-1.98000$

$-2.57000$

0.05900

0.14900

0.62800

$-1.41100$

$-2.11100$

$-1.71900$

$-1.09500$

$-1.69800$

$-1.77100$

$-0.19100$

0.36200

0.02700

$-0.48800$

$\odot .72900$

$-0.07900$

2. 28200

2. 21700

2. 54900

3.07600

1.89800 
Transition State (1,4 addition to simple enone):

\begin{tabular}{|c|c|c|c|}
\hline C & ๑. ๑๑९७९ & $\odot . ๑ \odot \odot \odot \odot$ & $\odot . \odot \odot \odot \odot \odot$ \\
\hline C & 1.42700 & $\odot .0 \odot \odot \odot \odot$ & $\odot .00 \odot \odot \odot$ \\
\hline C & 2.25600 & 1.09300 & $\odot . \odot \odot \odot \odot \odot$ \\
\hline $\mathrm{H}$ & 3.30300 & $\odot .9720 \odot$ & $-\odot .2440 \odot$ \\
\hline H & 1.85800 & 2.09500 & $-\odot .0980 \odot$ \\
\hline B & $-\odot .59700$ & 1.67300 & -1.70400 \\
\hline 0 & -1.09100 & 2.92900 & -1.99700 \\
\hline 0 & -0.15600 & 0.99200 & -2.81900 \\
\hline $\mathrm{Pd}$ & -0.59400 & $-0.0230 \odot$ & 2.11800 \\
\hline $\mathrm{P}$ & -2.95300 & $\odot .30 \odot \odot \odot$ & 1.96800 \\
\hline C & -4.05700 & -1.18600 & 1.88500 \\
\hline $\mathrm{H}$ & -3.82900 & $-1.8600 \odot$ & 2.71400 \\
\hline H & -5.11500 & -0.91100 & 1.93400 \\
\hline H & -3.88000 & -1.73200 & 0.95600 \\
\hline C & -3.68700 & 1.37200 & $\odot .65500$ \\
\hline $\mathrm{H}$ & -3.57500 & $\odot .89500$ & -0.32000 \\
\hline H & -4.75000 & 1.55700 & $\odot .83800$ \\
\hline $\mathrm{H}$ & -3.15200 & 2.32100 & 0.61600 \\
\hline C & -3.59900 & 1.12300 & 3.49800 \\
\hline H & -3.12900 & 2.10100 & 3.61300 \\
\hline H & -4.68500 & 1.25500 & 3.46000 \\
\hline H & -3.34800 & 0.52500 & 4.37700 \\
\hline C & -0.21700 & 1.89800 & -3.93000 \\
\hline $\mathrm{H}$ & 0.80100 & 2.21000 & -4.18400 \\
\hline H & -0.65100 & 1.38500 & -4.79000 \\
\hline C & -1.08200 & 3.07400 & -3.42300 \\
\hline $\mathrm{H}$ & -0.67000 & 4.05100 & -3.68300 \\
\hline $\mathrm{H}$ & -2.11200 & 3.01900 & -3.79100 \\
\hline C & 1.29800 & -0.13000 & 3.14900 \\
\hline $\mathrm{H}$ & 1.89000 & -1.01300 & 2.91000 \\
\hline H & $\odot .69500$ & $-0.2620 \odot$ & 4.06100 \\
\hline C & 1.93200 & 1.12600 & 2.99400 \\
\hline $\mathrm{H}$ & 1.42200 & 1.99800 & 3.40000 \\
\hline C & 3.05200 & 1.33500 & 2.22100 \\
\hline H & 3.71700 & 0.50900 & 1.99800 \\
\hline H & 3.47700 & 2.32700 & 2.11200 \\
\hline 0 & -0.60500 & 1.21300 & -0.43100 \\
\hline $\mathrm{H}$ & 1.87800 & -0.98800 & 0.05700 \\
\hline C & -0.67700 & -1.23100 & -0.58500 \\
\hline H & -0.49900 & -1.27600 & -1.66600 \\
\hline $\mathrm{H}$ & $-\odot .28100$ & -2.14100 & -0.13100 \\
\hline H & -1.75500 & -1.21300 & -0.42300 \\
\hline
\end{tabular}

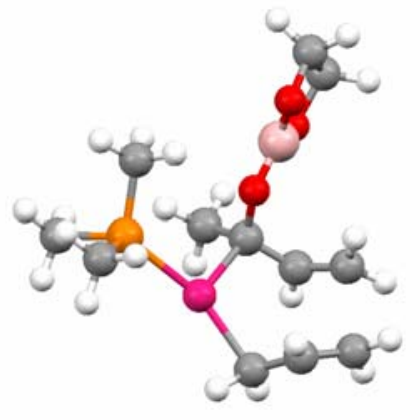

\section{REFERENCES}

(1) A. D. Becke, J. Chem. Phys. 98, 1372 (1993).

(2) C. Lee, W. Yang, and R. G. Parr, Phys. Rev. B 37, 785(1988).

(3) Bergner A, Dolg M, Kuechle W, Stoll H, Preuss H. Mol. Phys. 80, 1431(1993).

(4) R. Krishnan, J. S. Binkley, R. Seeger and J. A. Pople, J. Chem. Phys. 72, 650 (1980).

(5) M. J. Frisch, G. W. Trucks, H. B. Schlegel, G. E. Scuseria, M. A. Robb, J. R. Cheeseman, J. A. Montgomery, Jr., T. Vreven, K. N. Kudin, J. C. Burant, J. M. Millam, S. S. Iyengar, J. Tomasi, V. Barone, B. Mennucci, M. Cossi, G. Scalmani, N. Rega, G. A. Petersson, H. Nakatsuji, M. Hada, M. Ehara, K. Toyota, R. Fukuda, J. Hasegawa, M. Ishida, T. Nakajima, Y. Honda, O. Kitao, H. 
Nakai, M. Klene, X. Li, J. E. Knox, H. P. Hratchian, J. B. Cross, C. Adamo, J. Jaramillo, R. Gomperts, R. E. Stratmann, O. Yazyev, A. J. Austin, R. Cammi, C. Pomelli, J. W. Ochterski, P. Y. Ayala, K. Morokuma, G. A. Voth, P. Salvador, J. J. Dannenberg, V. G. Zakrzewski, S. Dapprich, A. D. Daniels, M. C. Strain, O. Farkas, D. K. Malick, A. D. Rabuck, K. Raghavachari, J. B. Foresman, J. V. Ortiz, Q. Cui, A. G. Baboul, S. Clifford, J. Cioslowski, B. B. Stefanov, G. Liu, A. Liashenko, P. Piskorz, I. Komaromi, R. L. Martin, D. J. Fox, T. Keith, M. A. Al-Laham, C. Y. Peng, A. Nanayakkara, M. Challacombe, P. M. W. Gill, B. Johnson, W. Chen, M. W. Wong, C. Gonzalez, and J. A. Pople, Gaussian, Inc., Wallingford CT, 2004.

(6) http://its.unc.edu/hpc/hardware/\#Cedar/Cypress|outline 


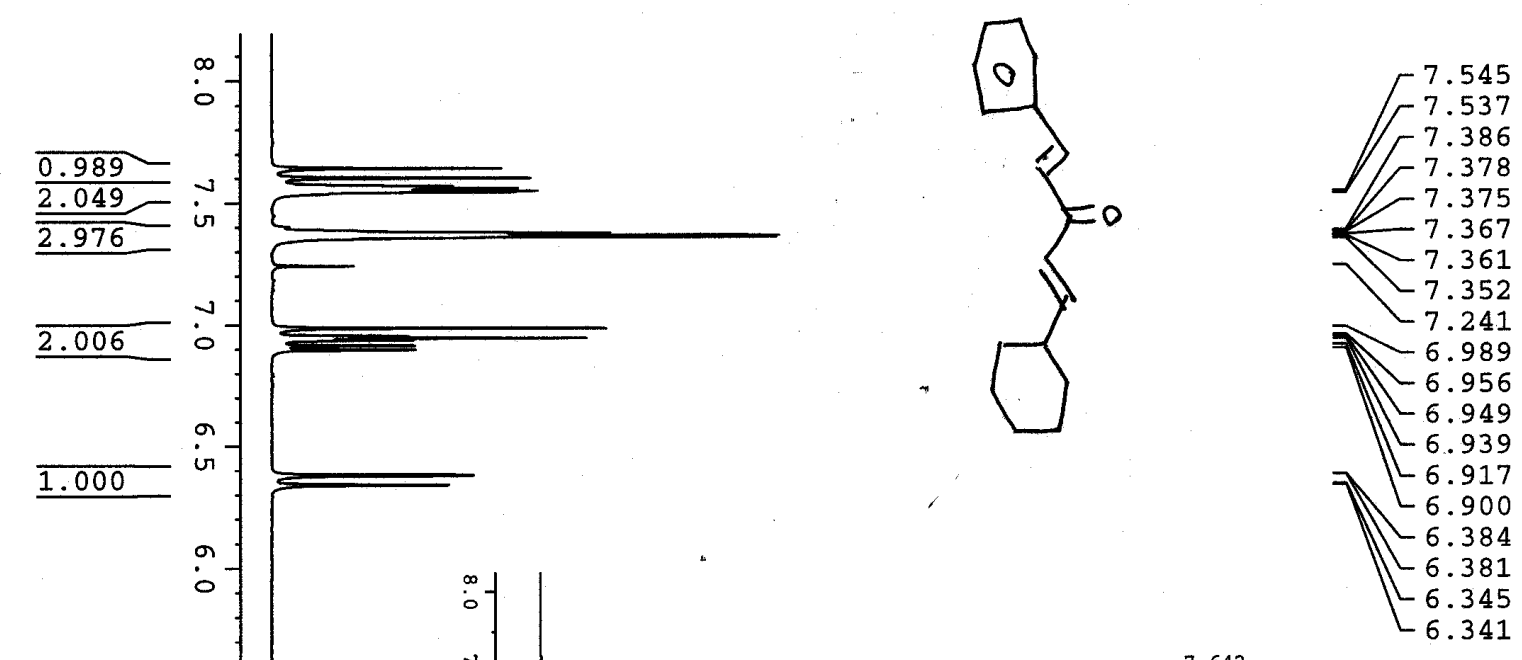

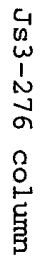



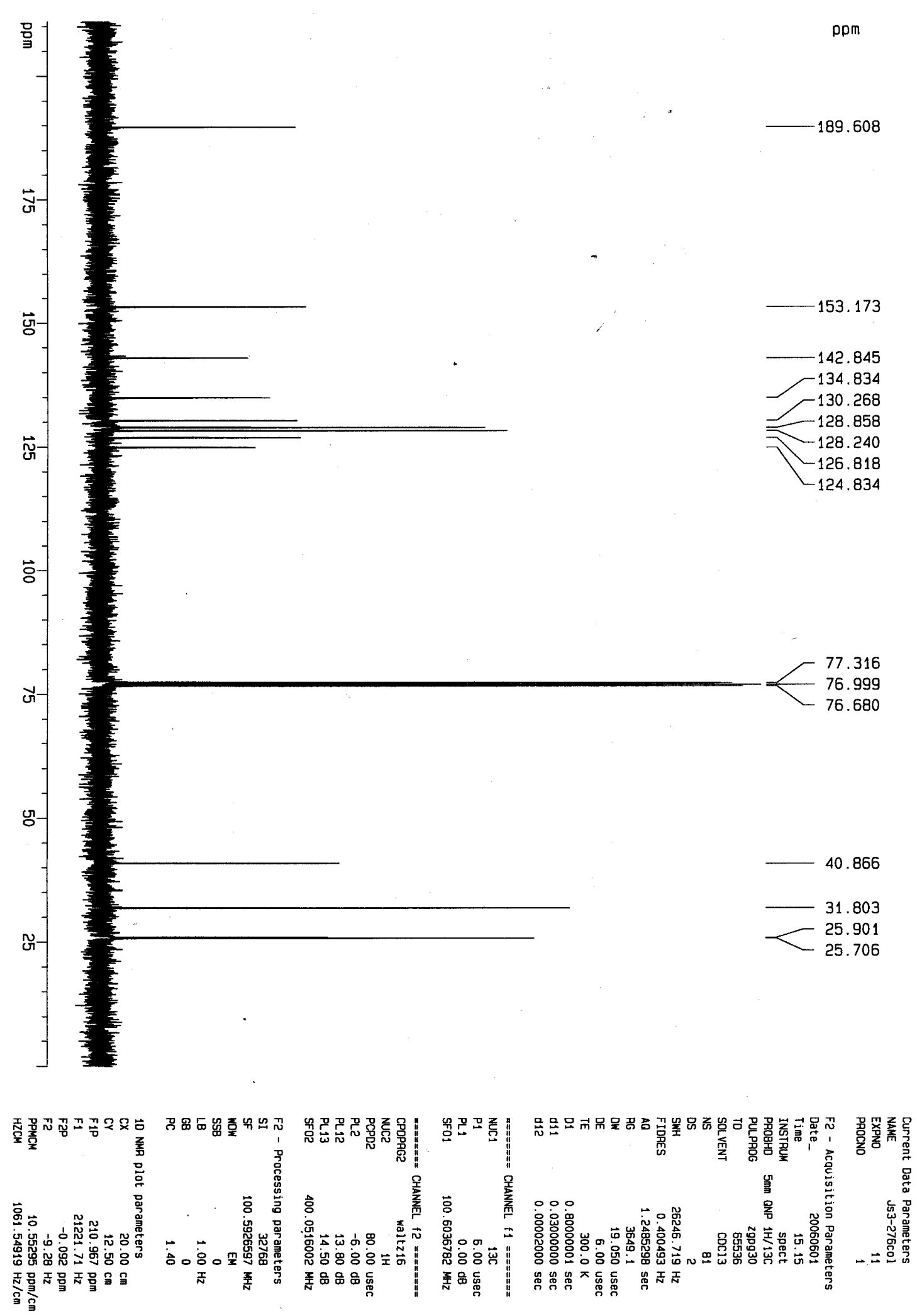


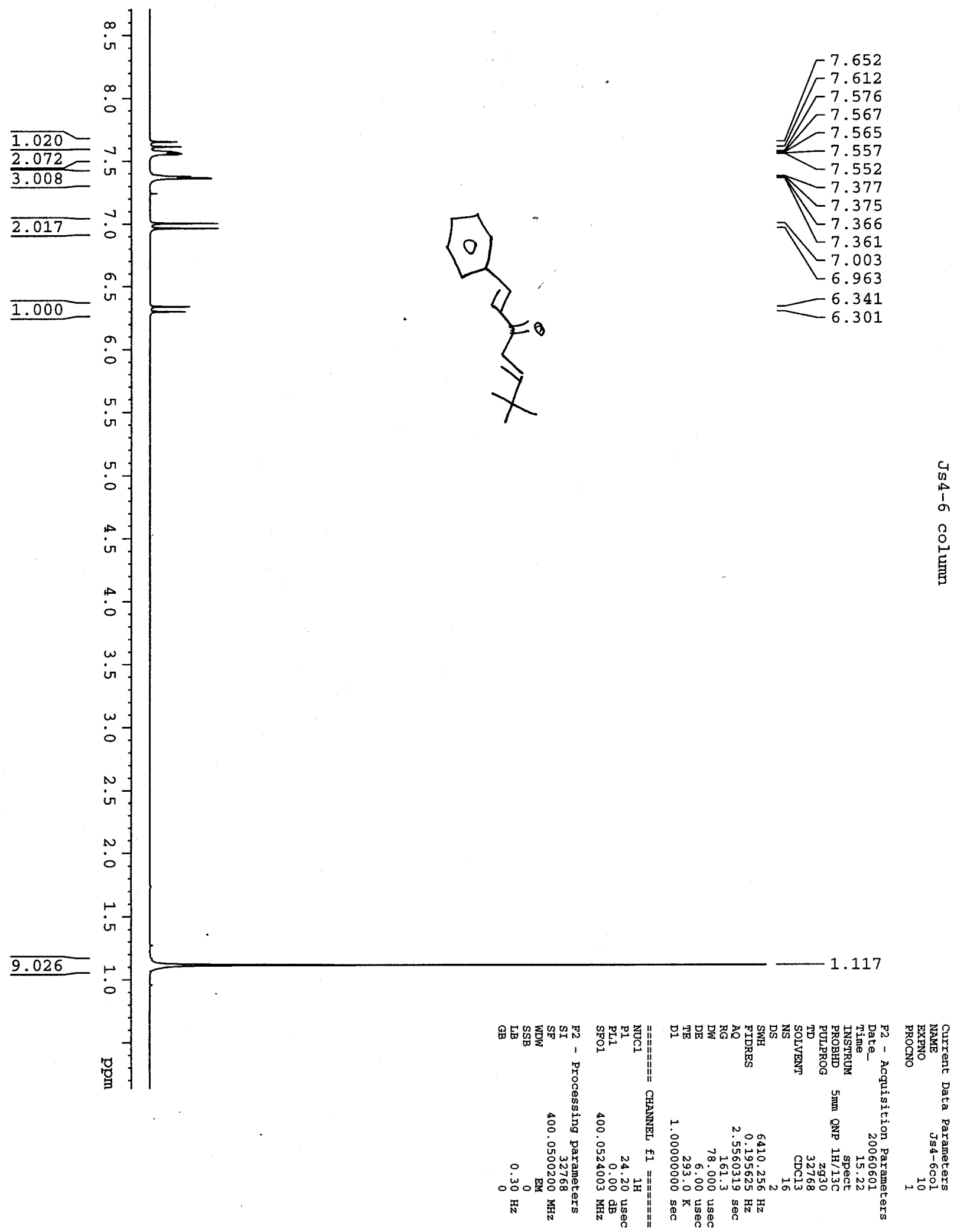

Page SI-22 

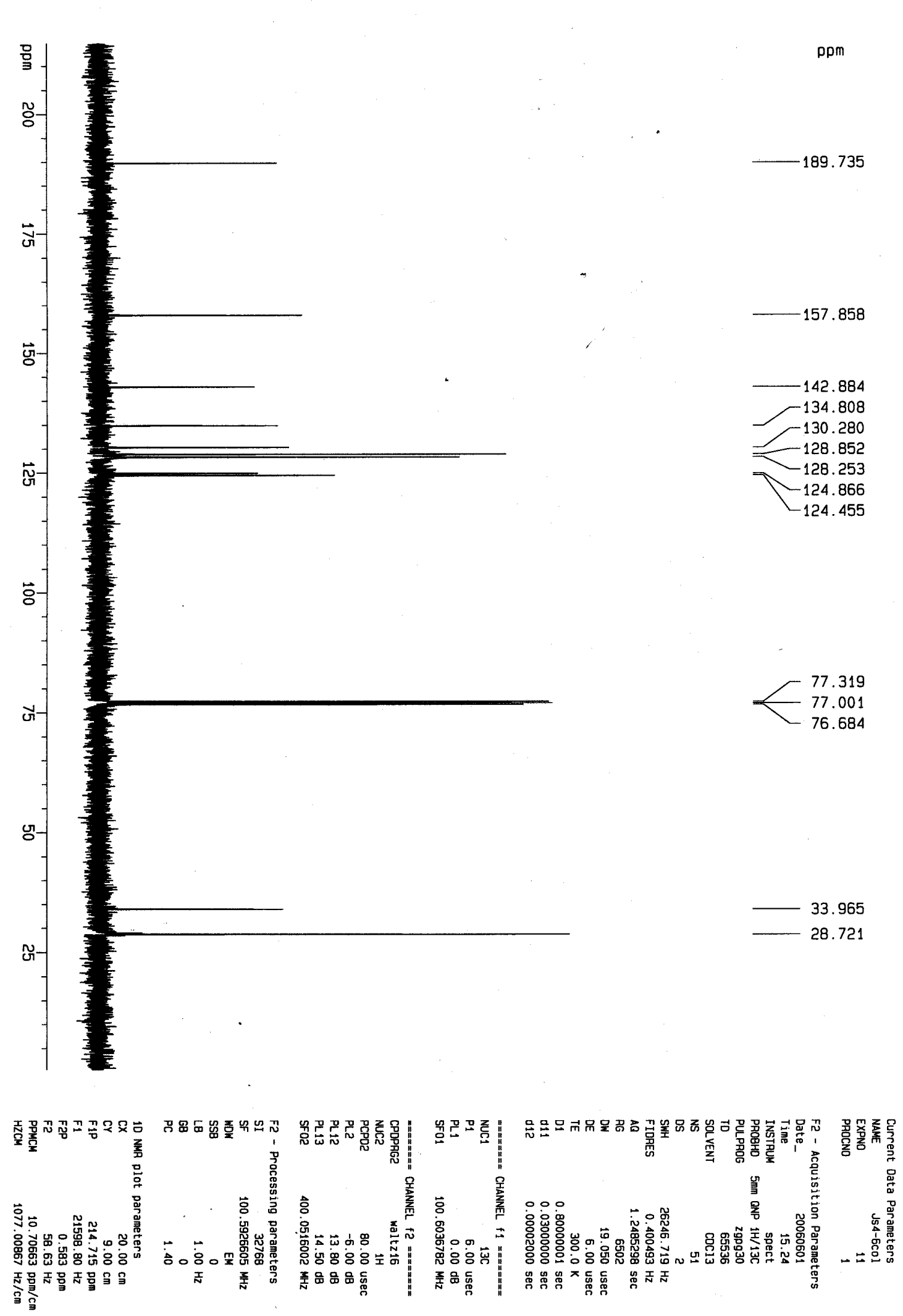


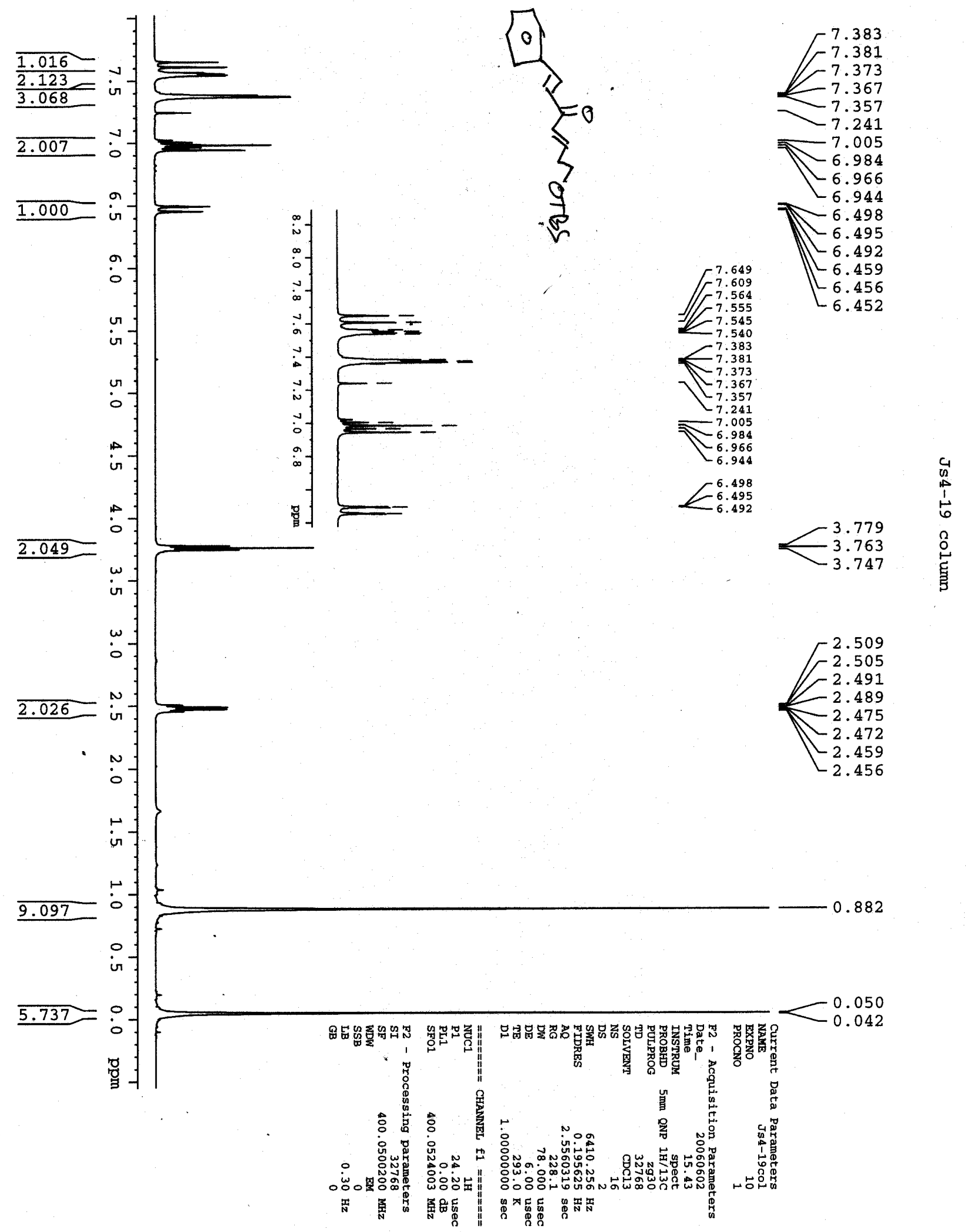

Page SI-24 

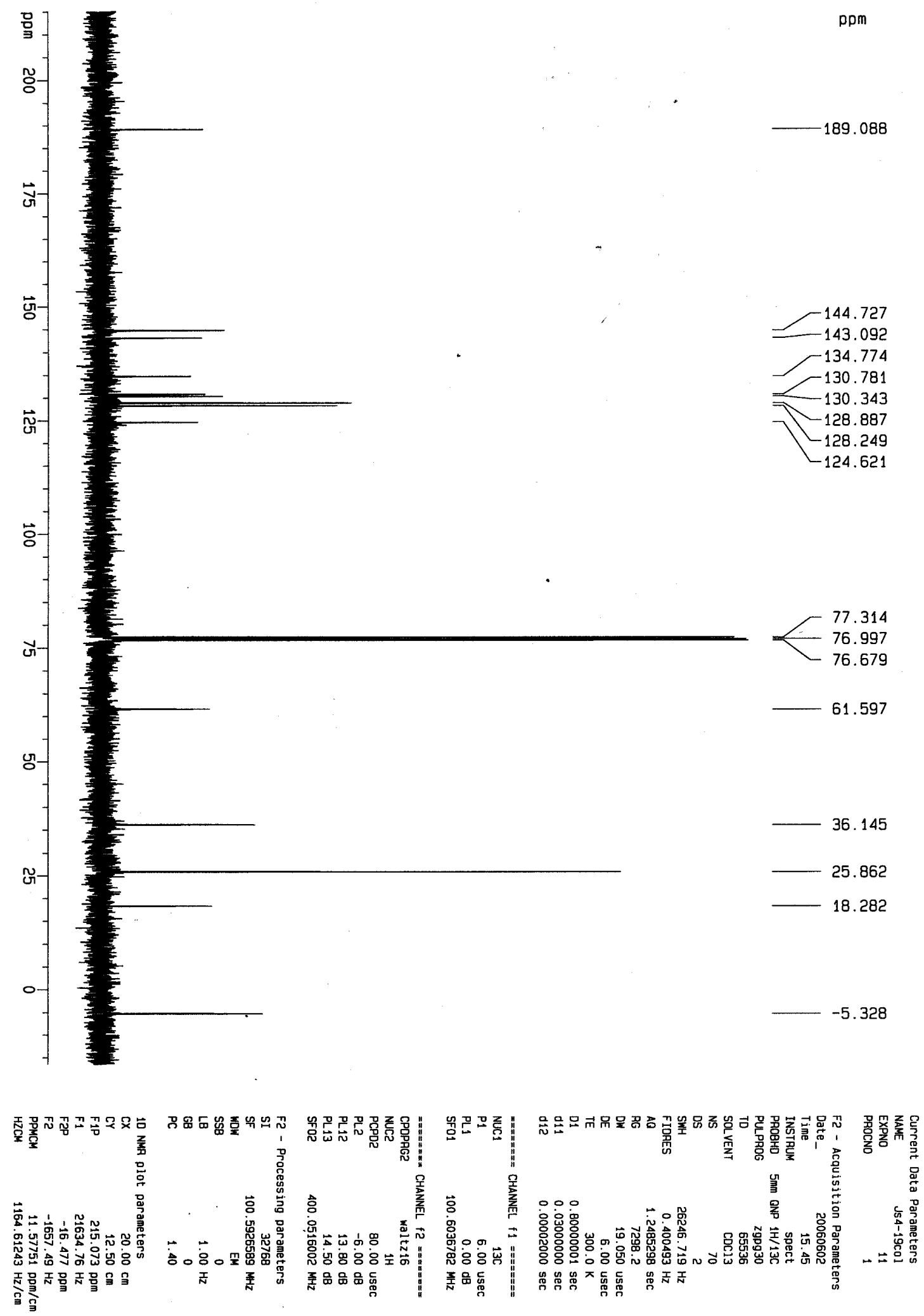


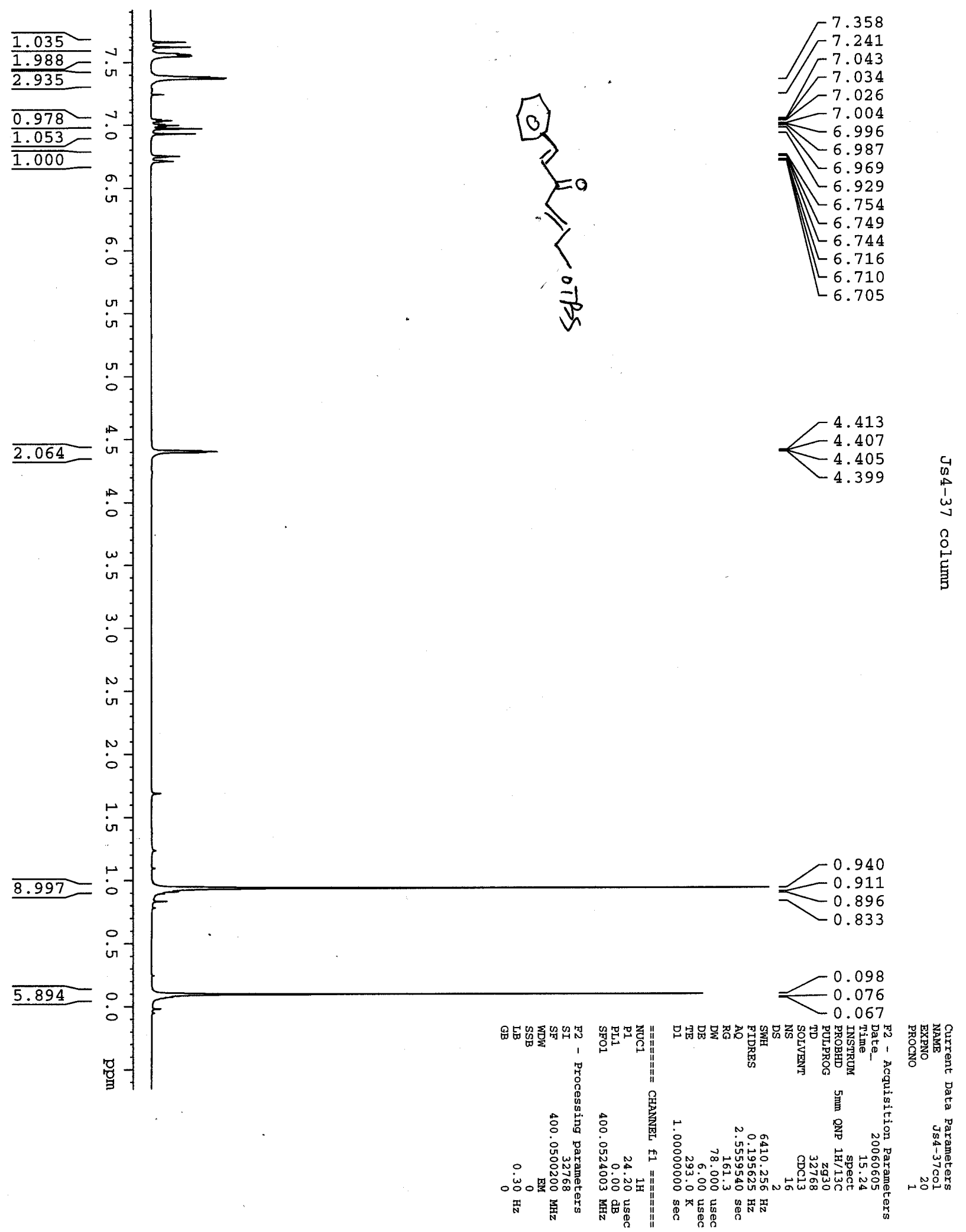

Page SI-26 

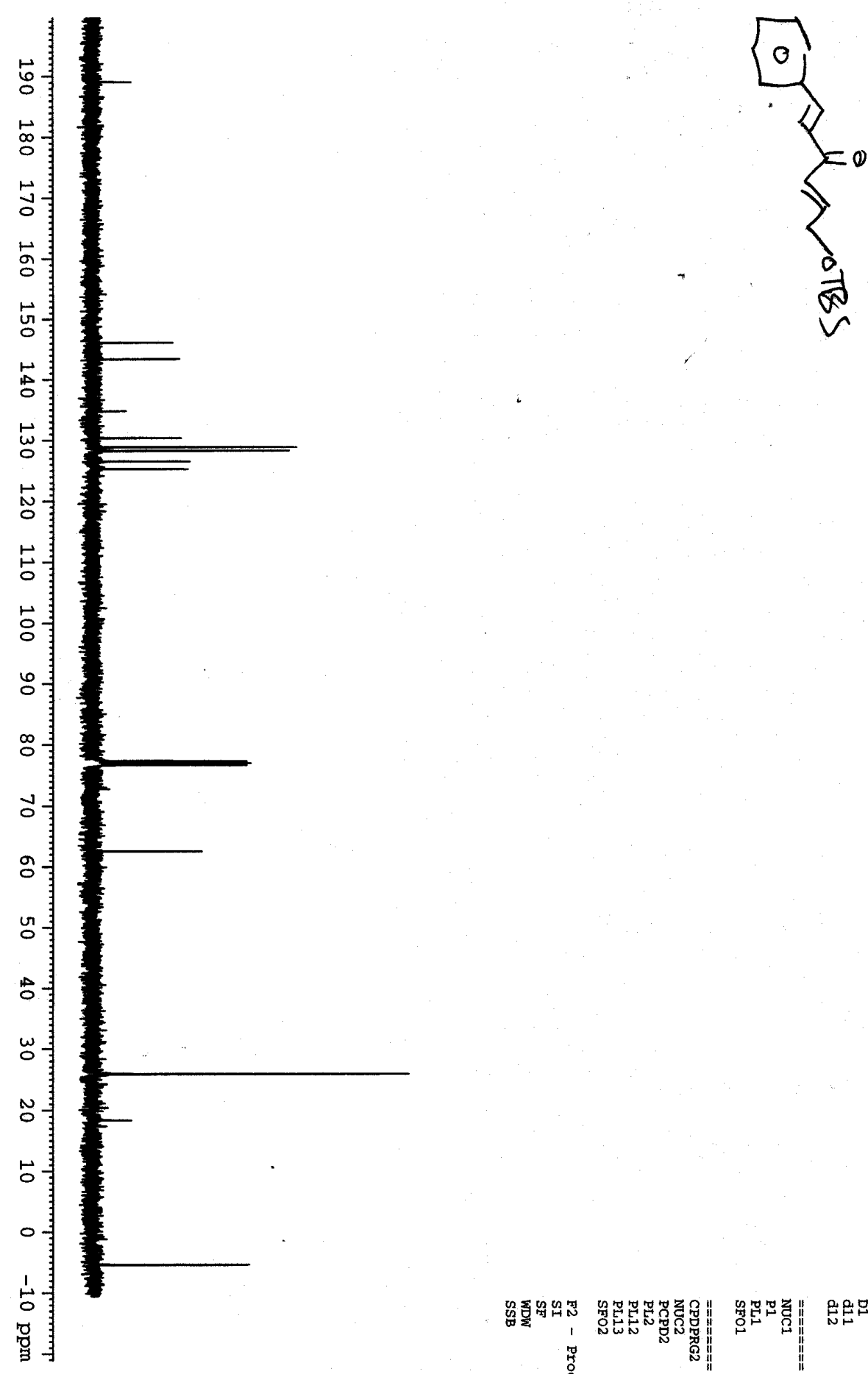

189.051

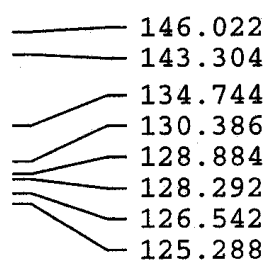

77.315

76.998

62.516

25.855

18.369

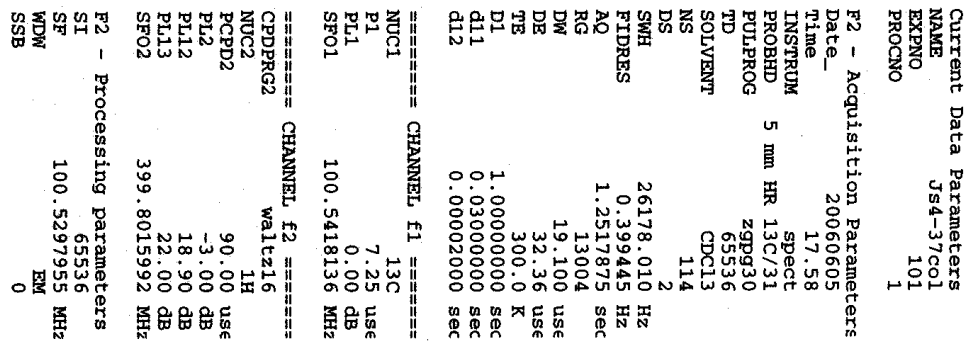

Page SI-27 


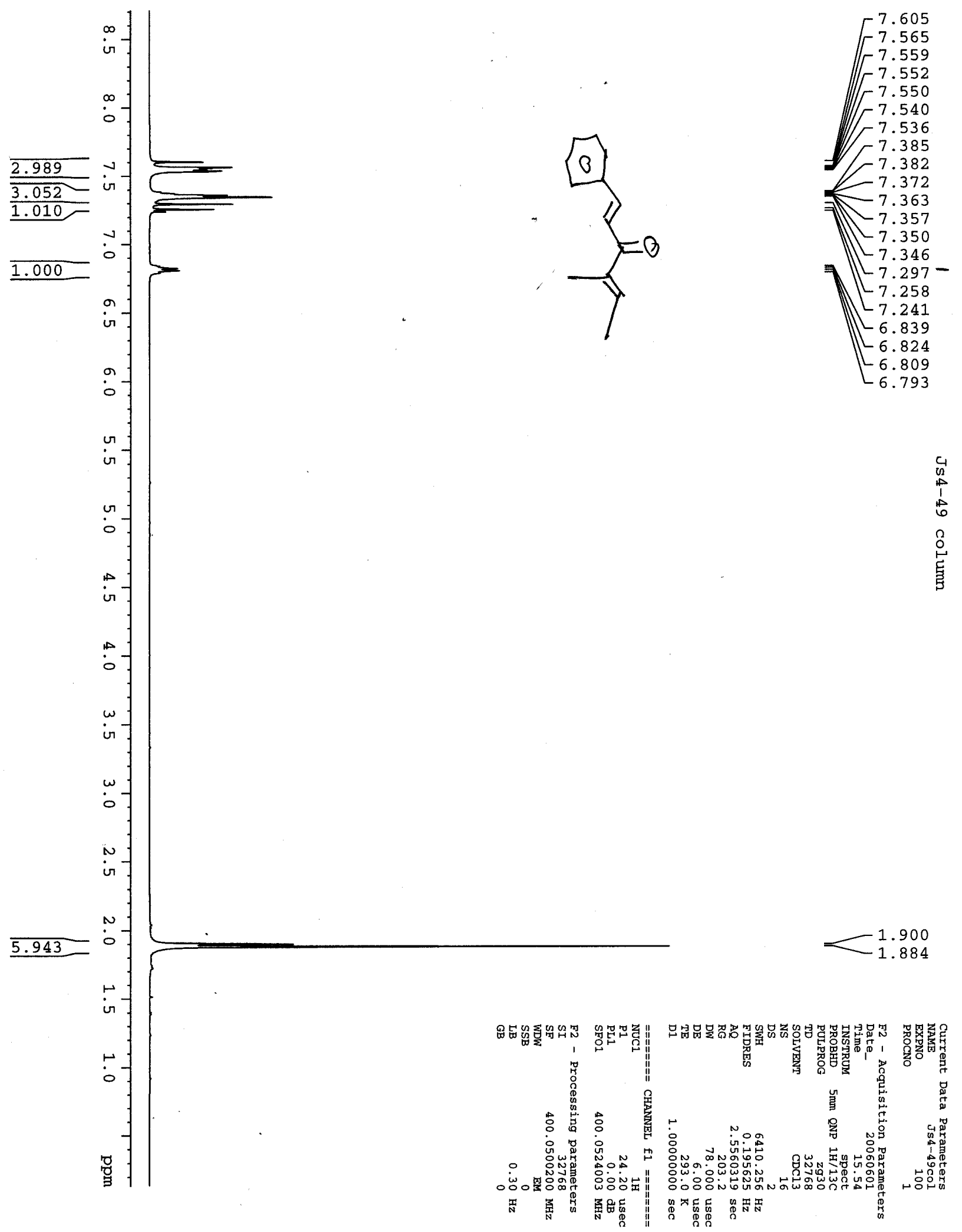

Page SI-28 


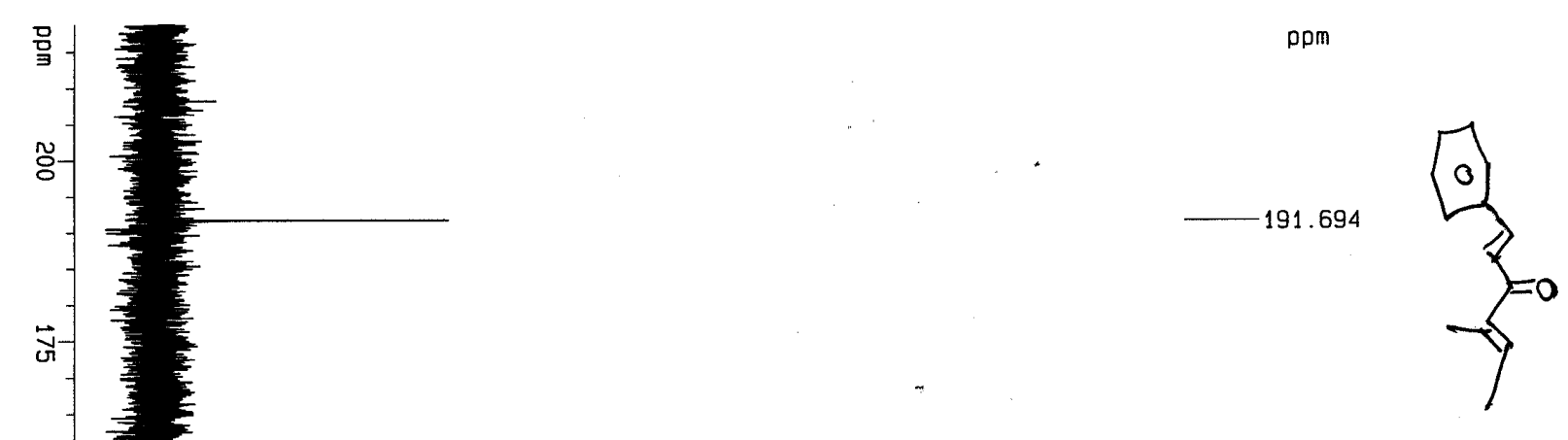

77.317

76.999

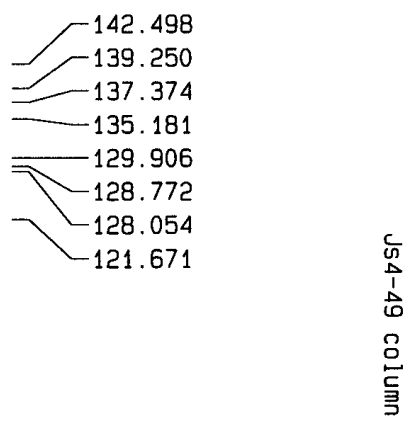

जै

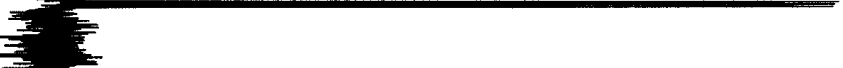

76.680

당-

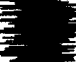

$=$

$-1$

ज-

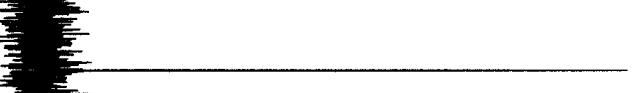

14.797

11.545

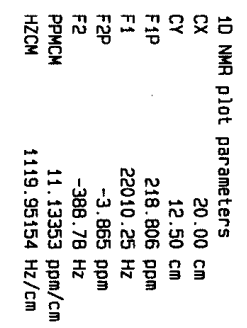

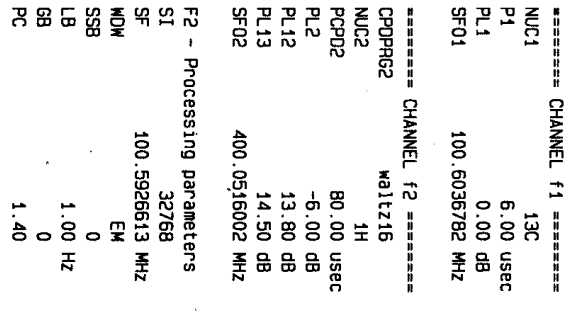

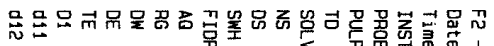

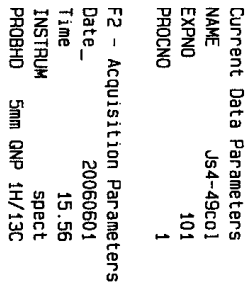



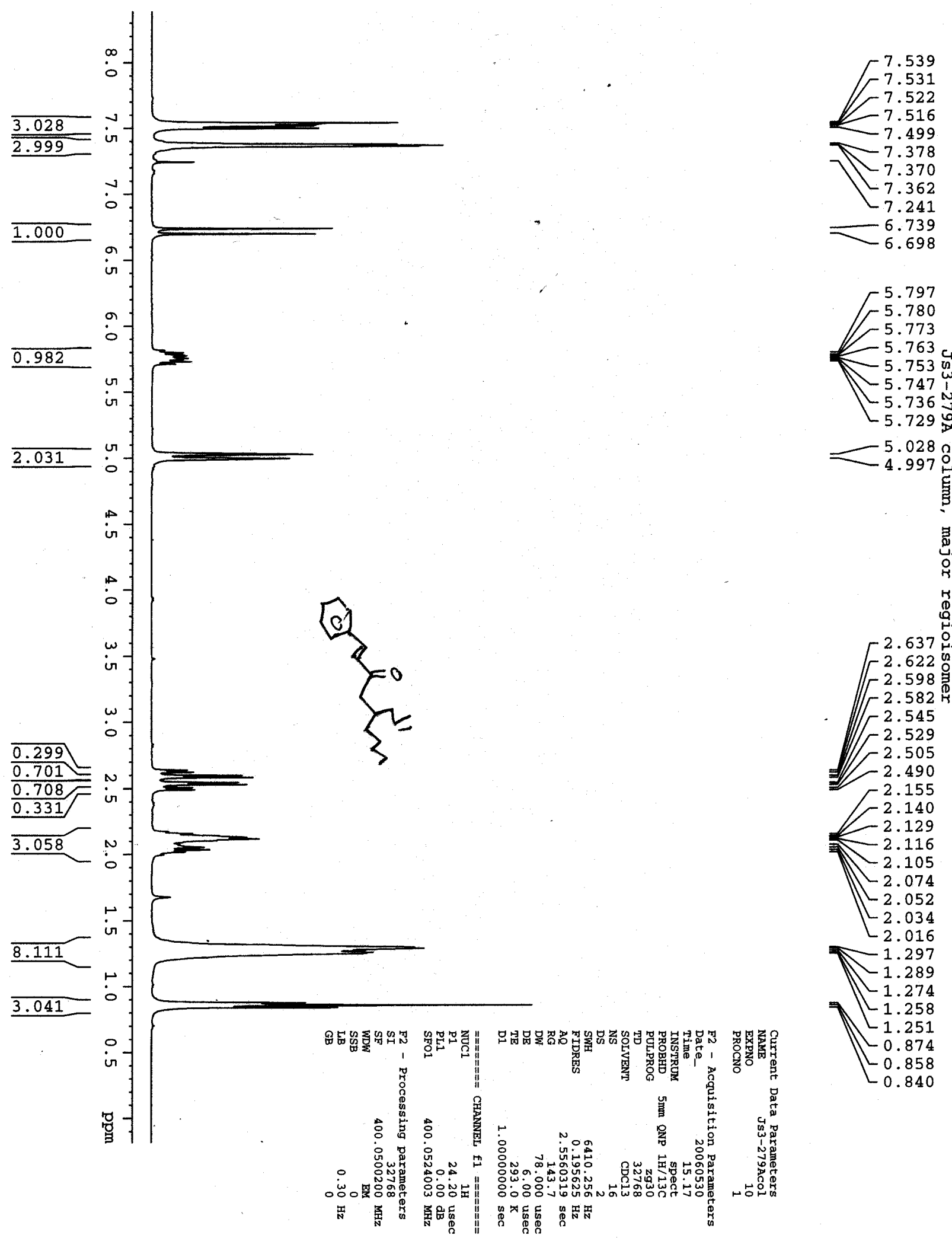

-2.622 \%

-2.598 当

- $-2.582 \stackrel{\mathbb{R}}{\mathrm{H}}$

$-2.545$

$-2.529$

2.505
-2.490

$-2.155$

$-2.140$

$-2.129$

2.116

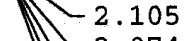

$-2.074$

$-2.052$

$-2.034$

2.016

1.297

$-1.289$

$-1.274$

$-1.258$

$\lcm{1.251}$

$-0.858$

$L_{0.840}$

Page SI-30 

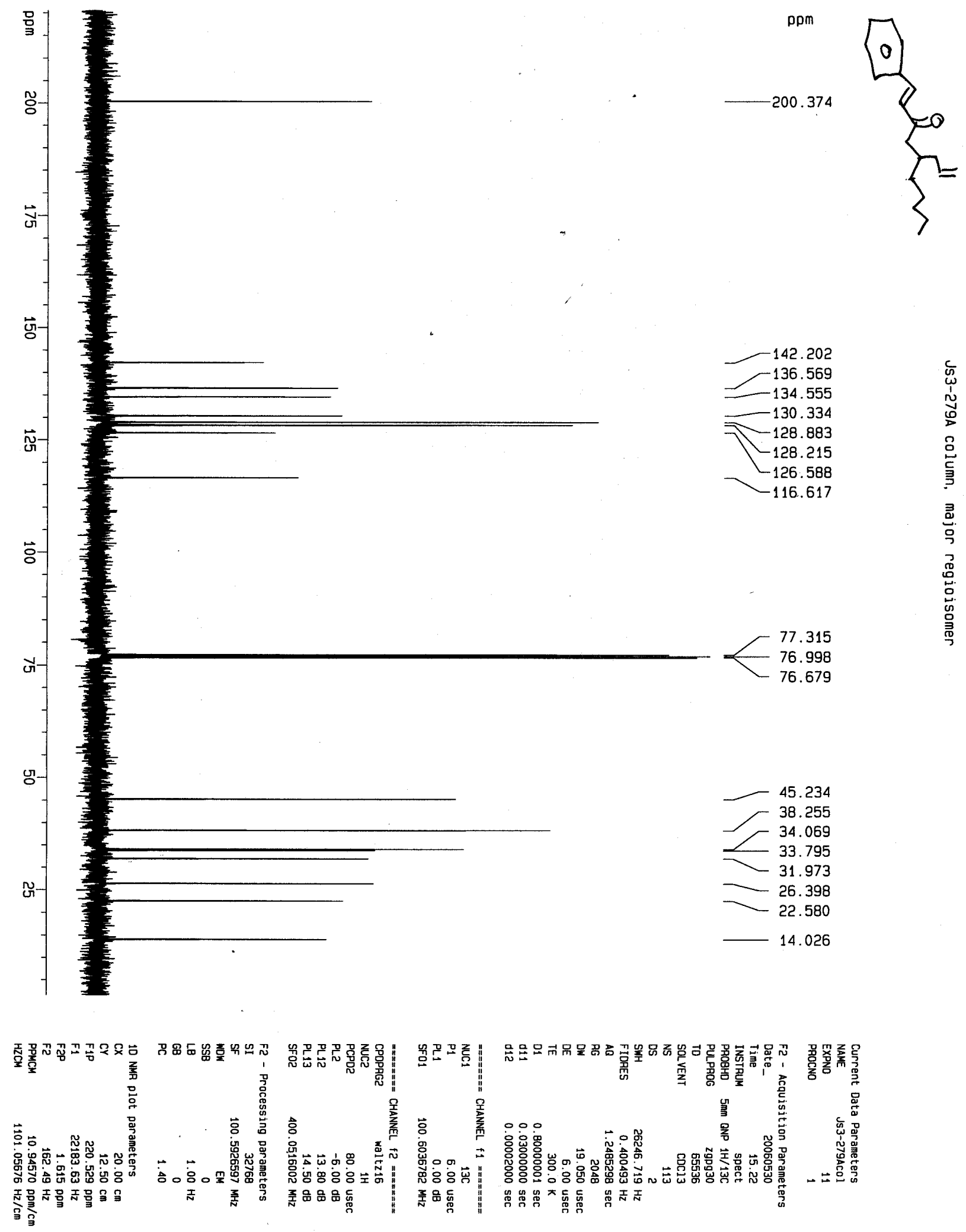

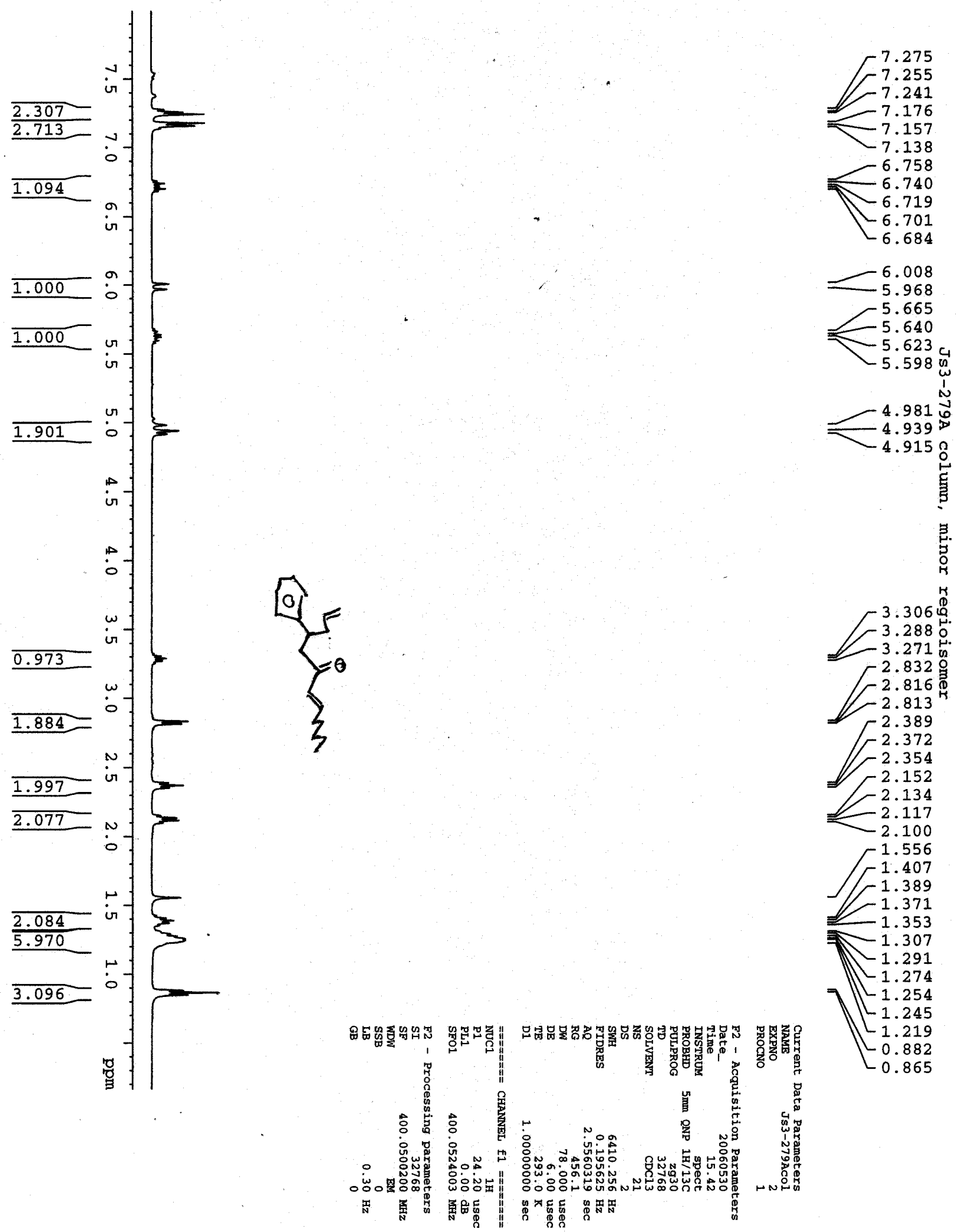

Page SI-32 

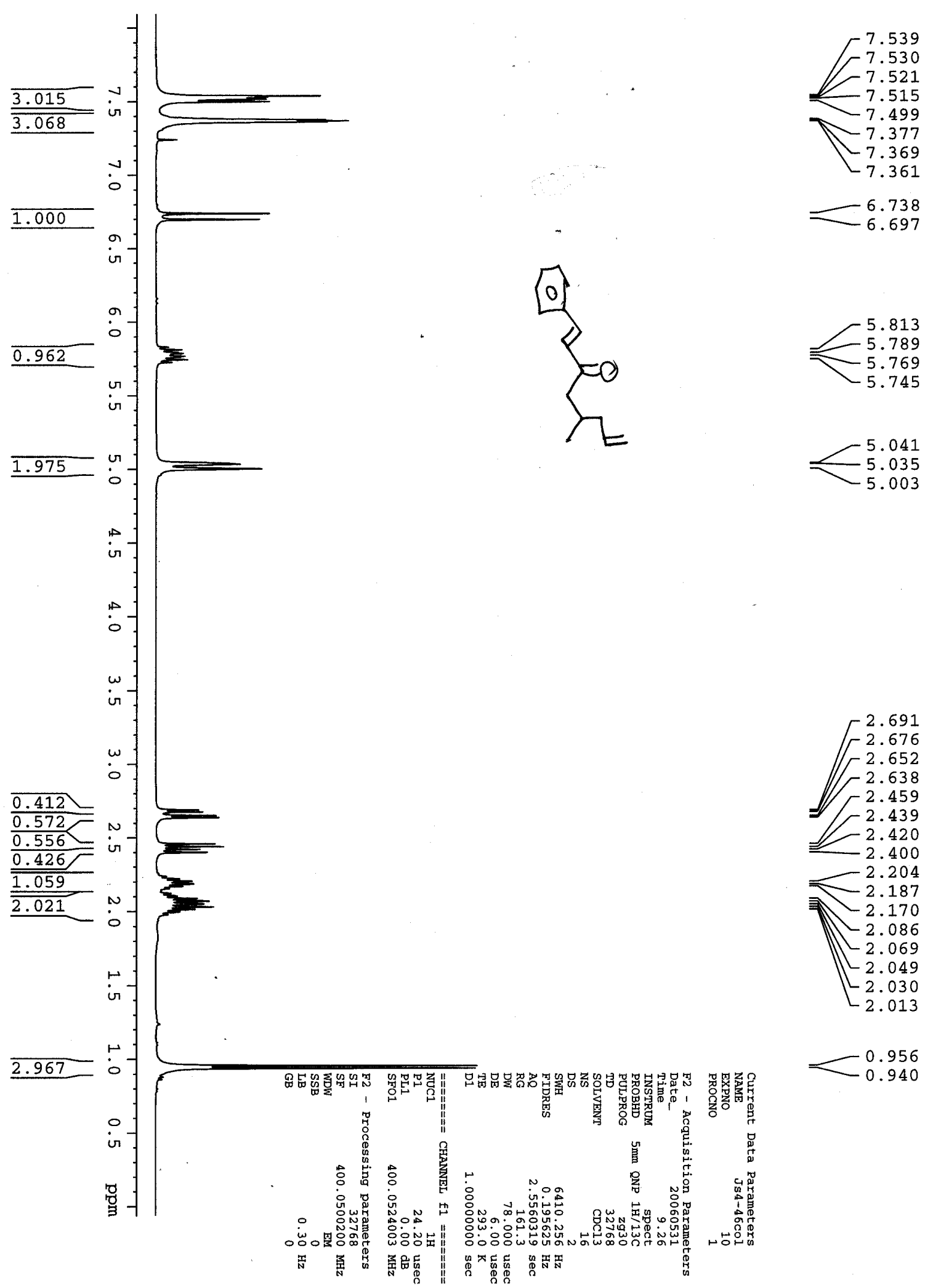

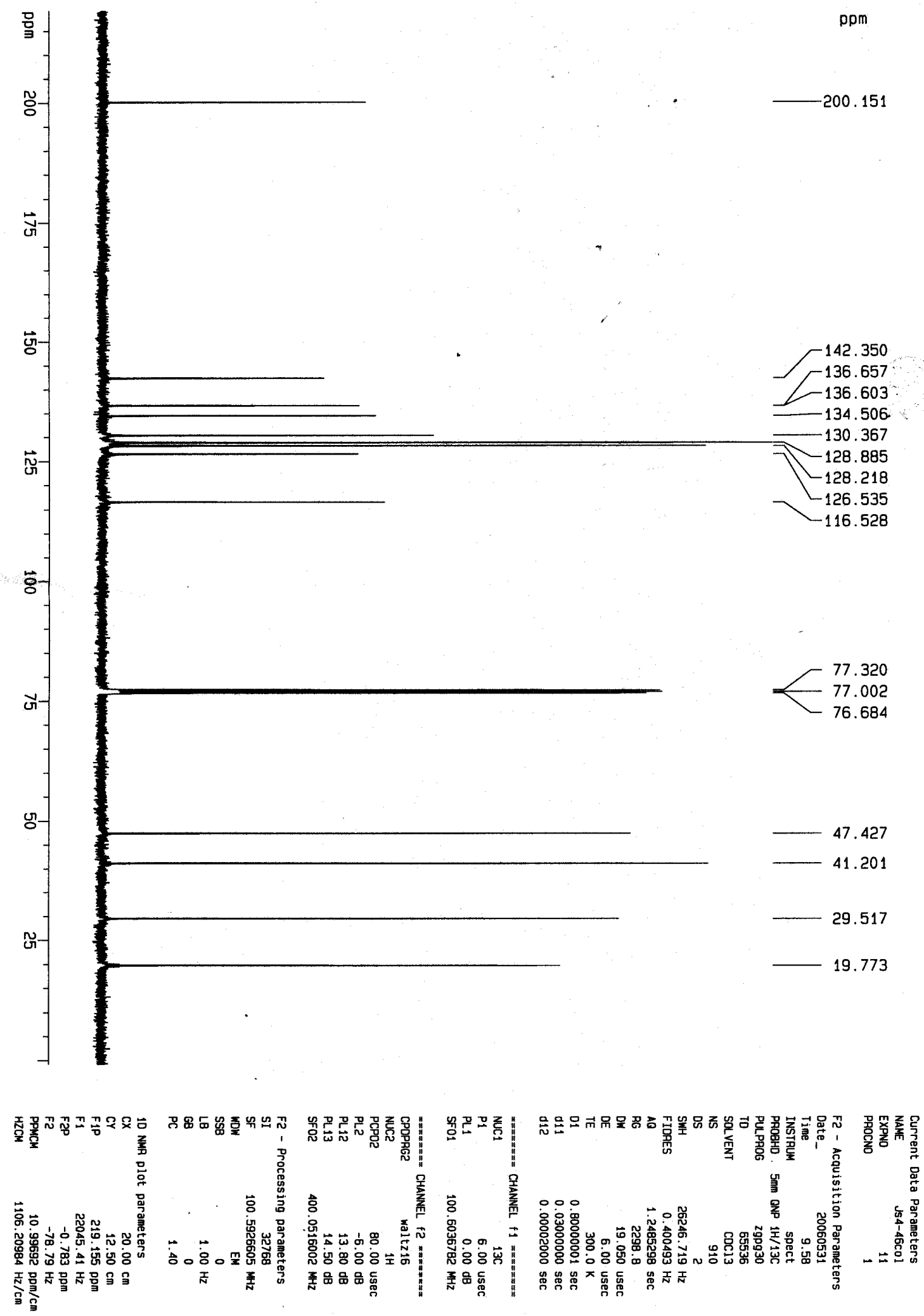

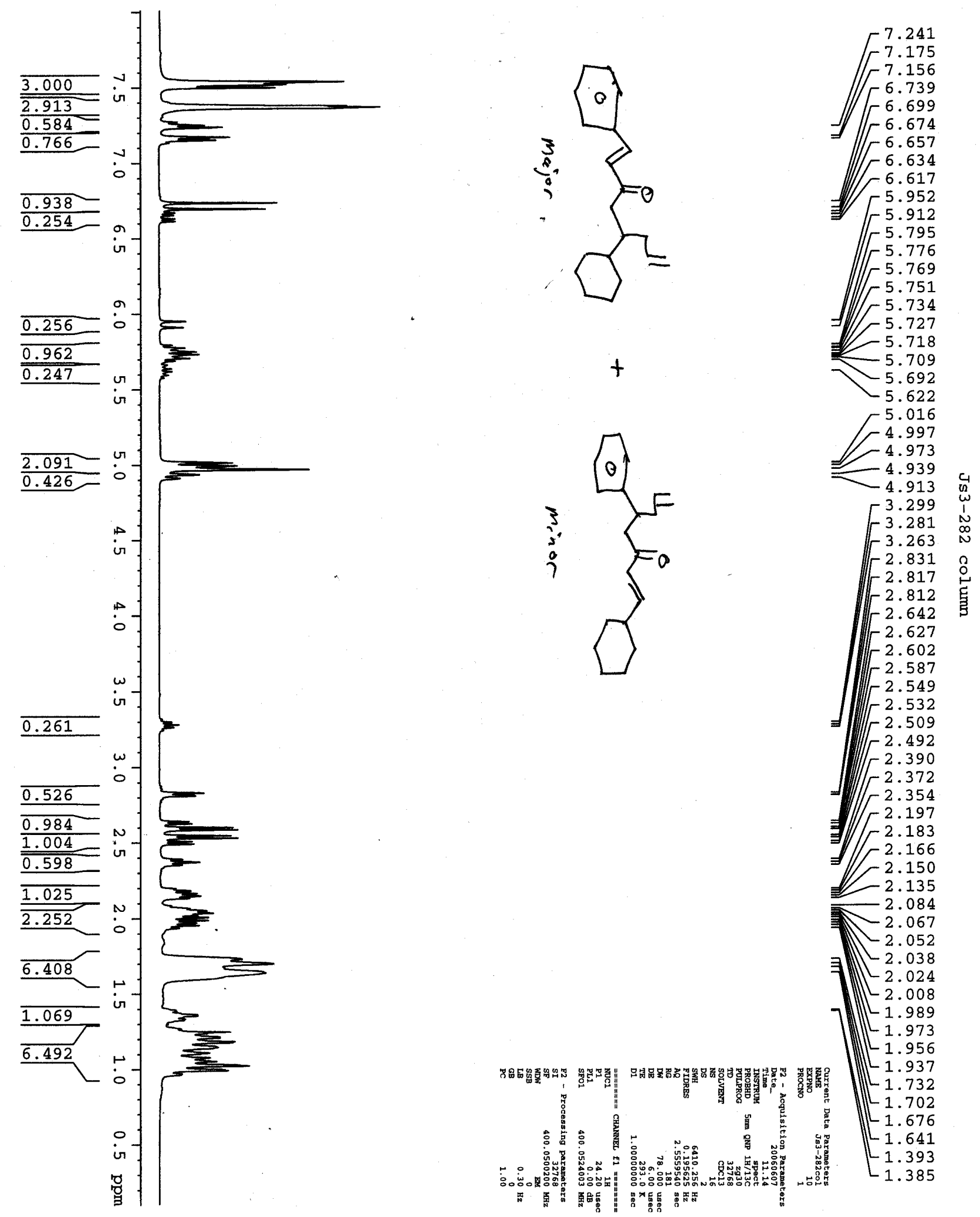


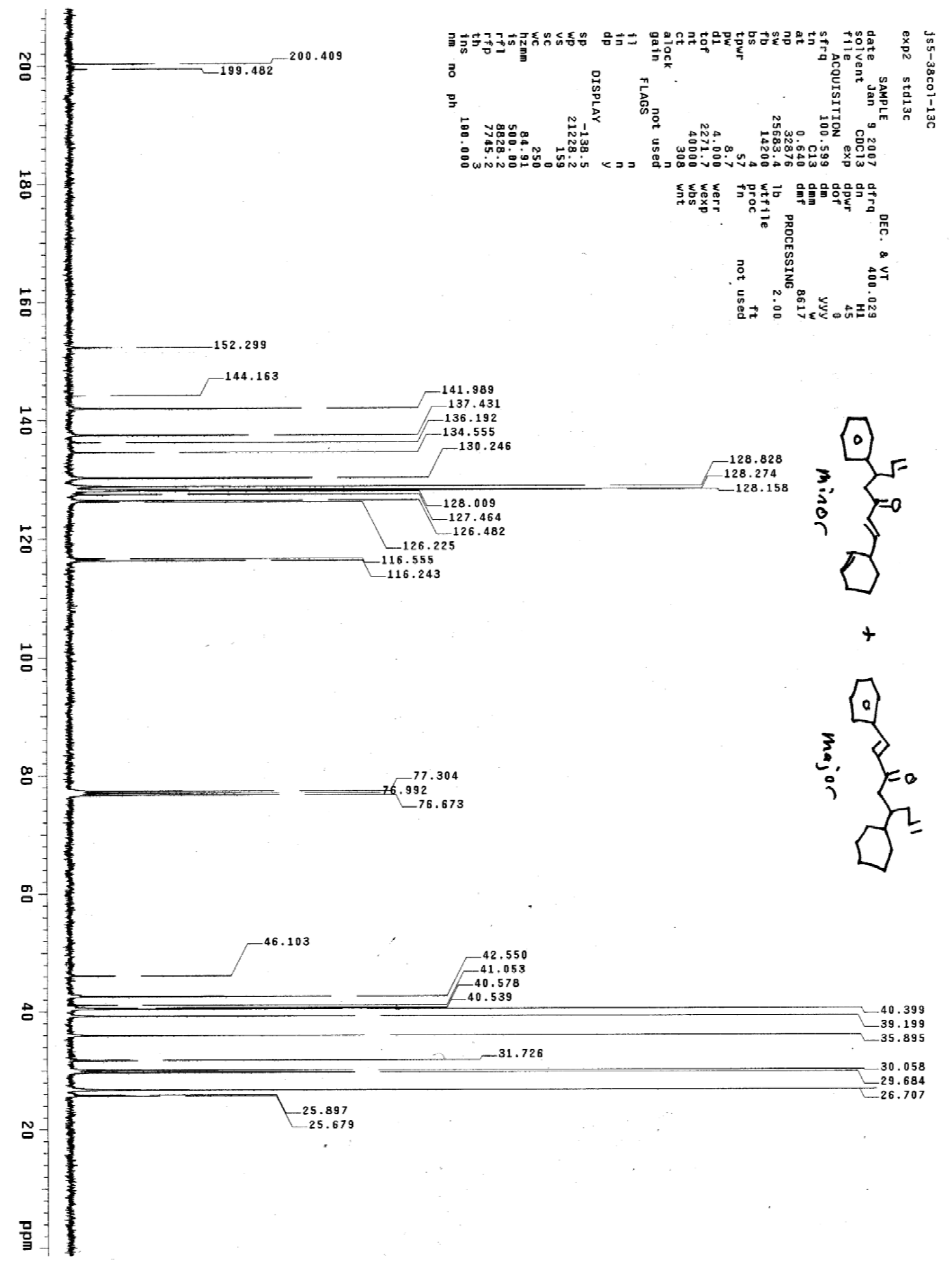




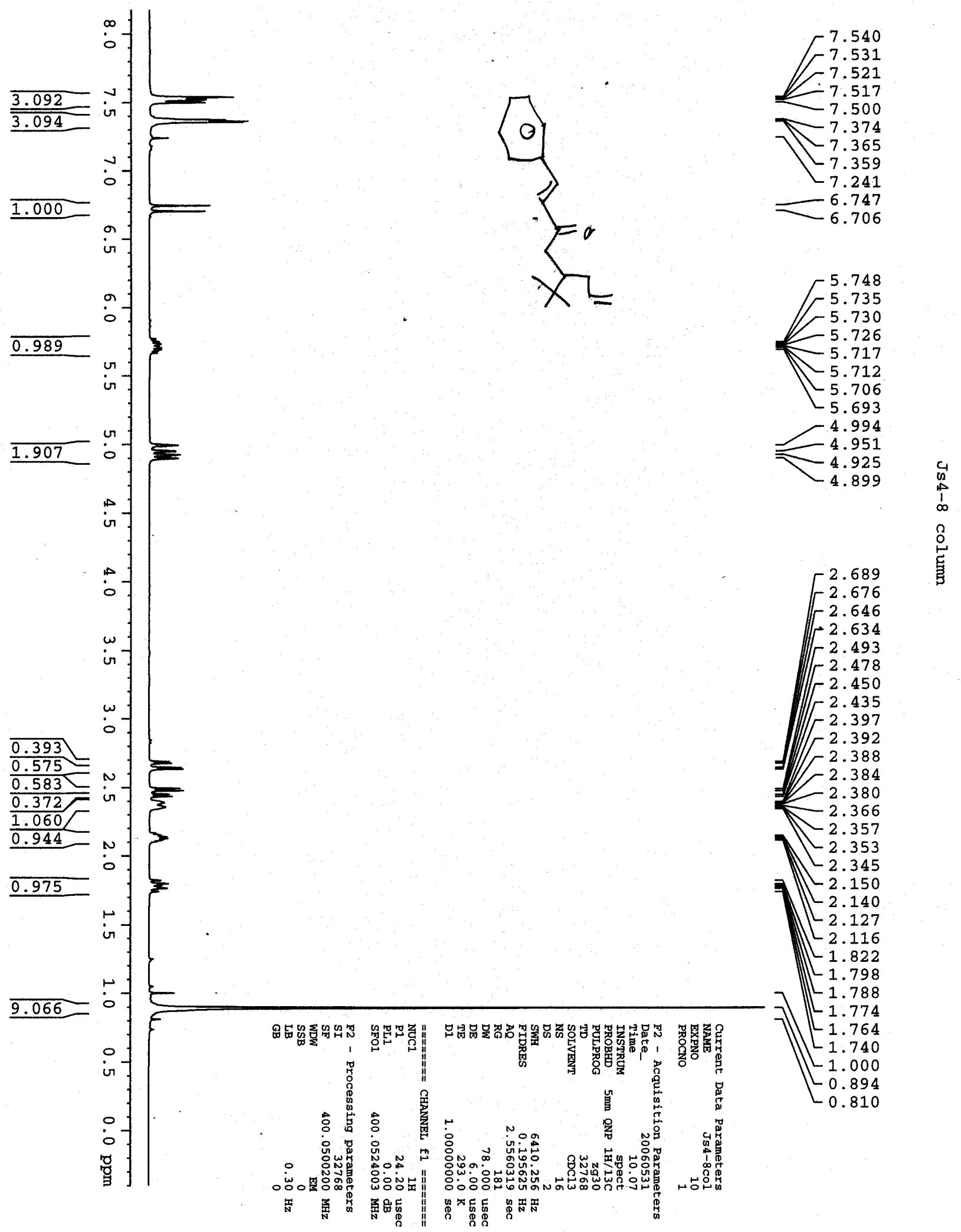

Page SI-37 

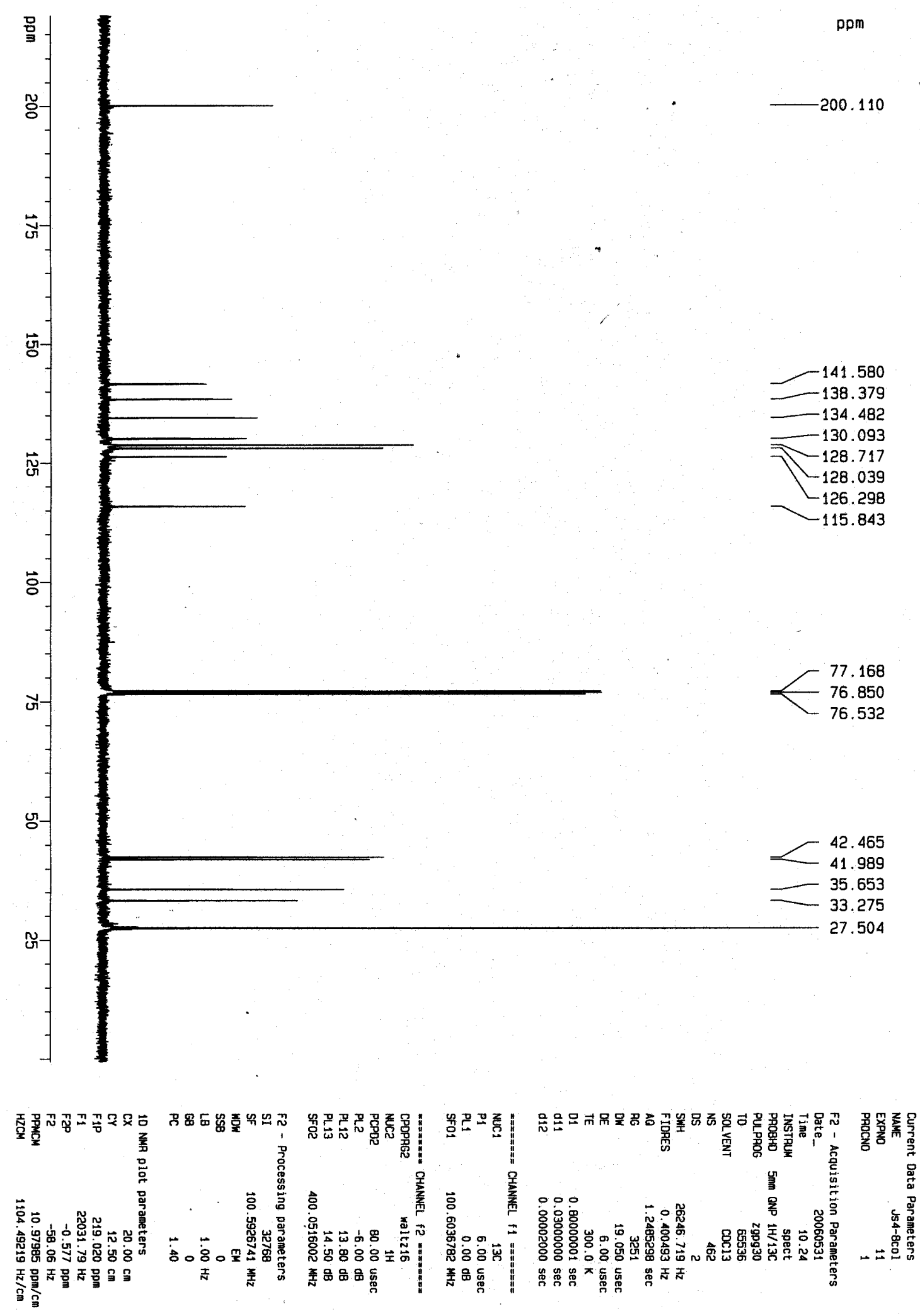

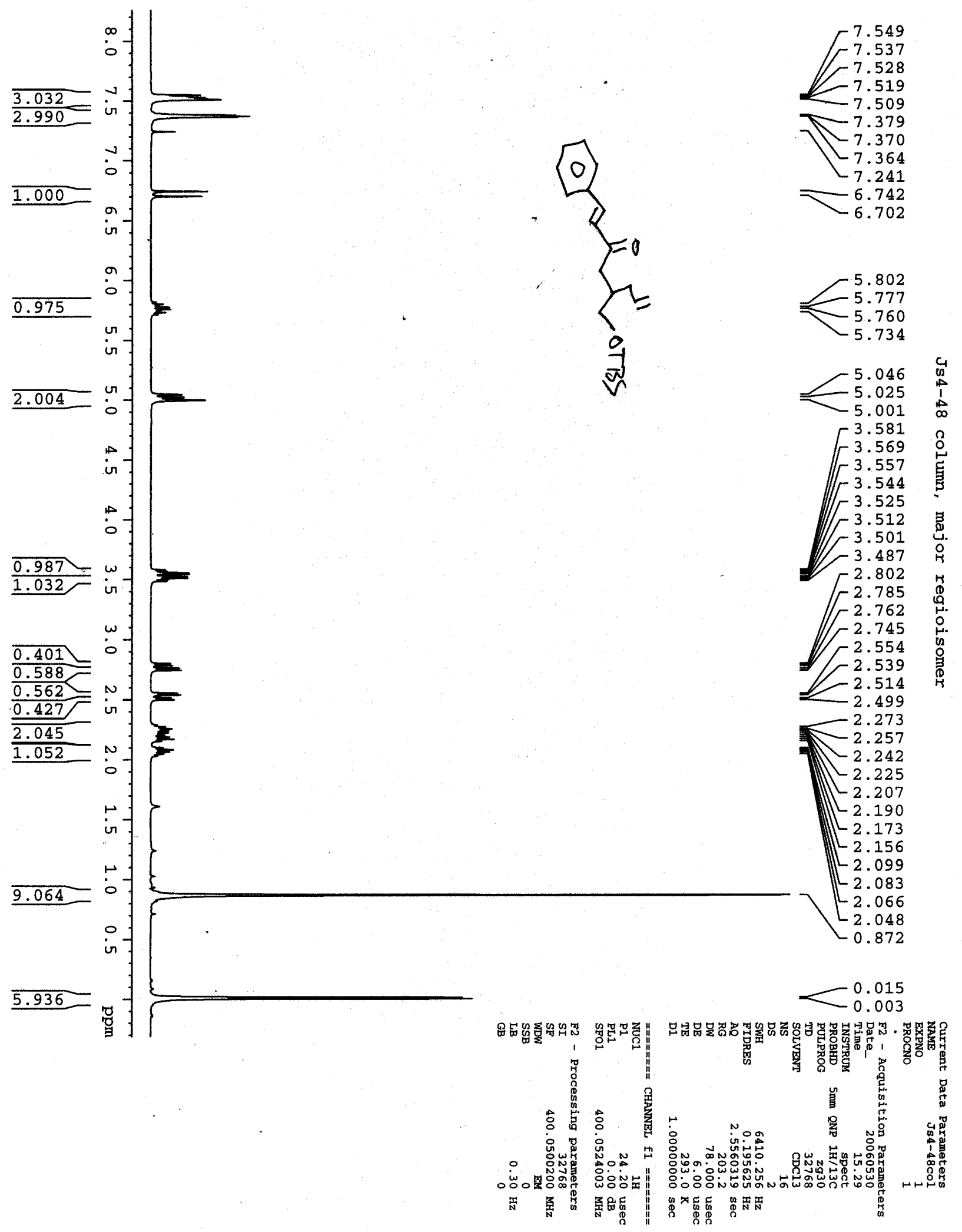

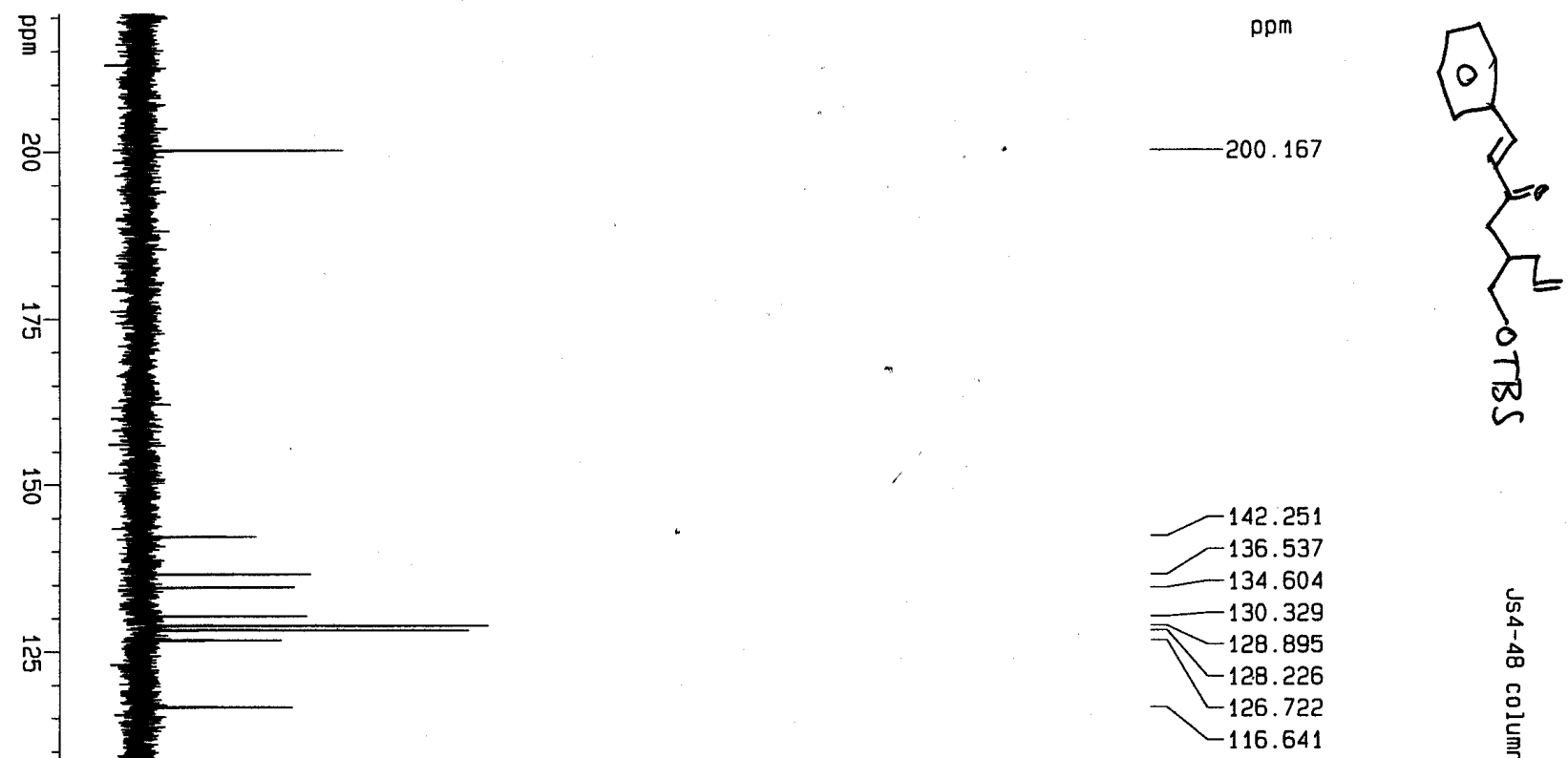

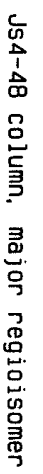

77.317

76.681

64.728

당

ษ
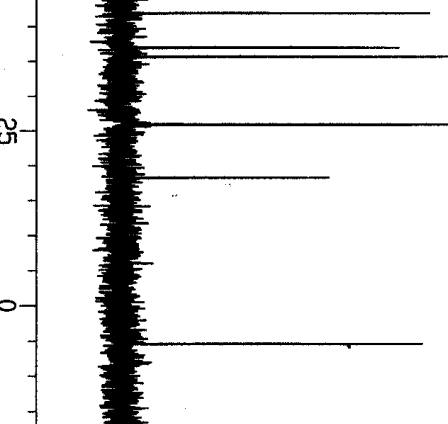

- 36.941

35.632

25.889

18.260

$-5.463$

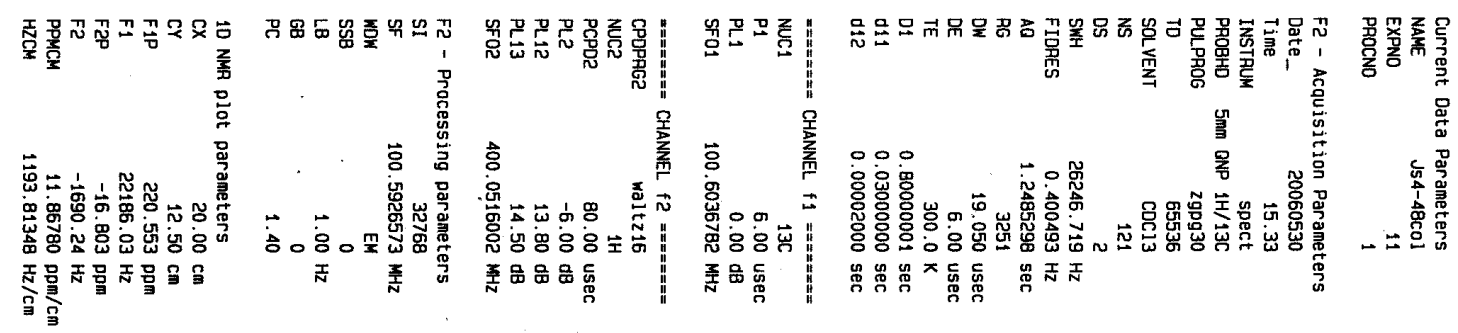




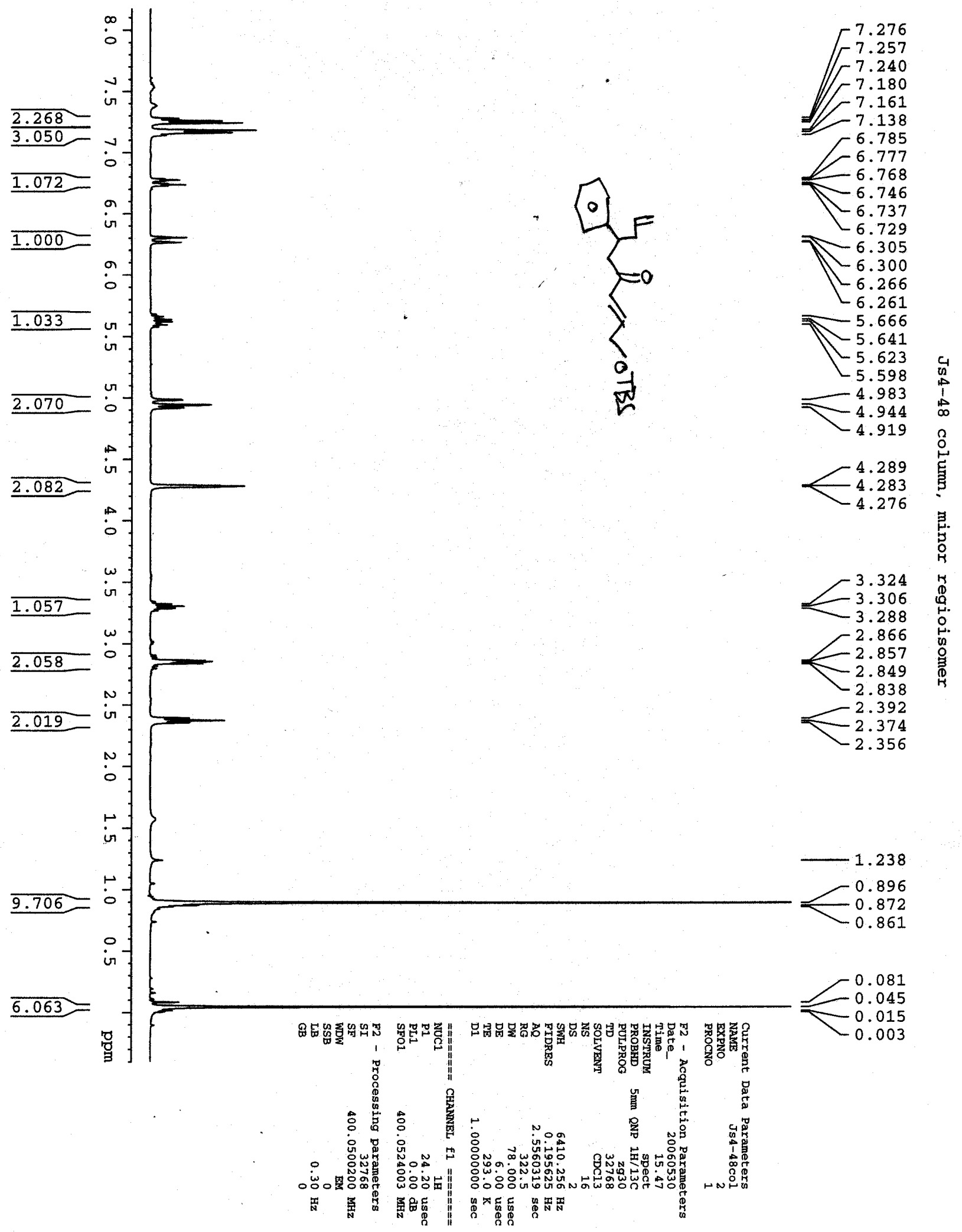

Page SI-41 

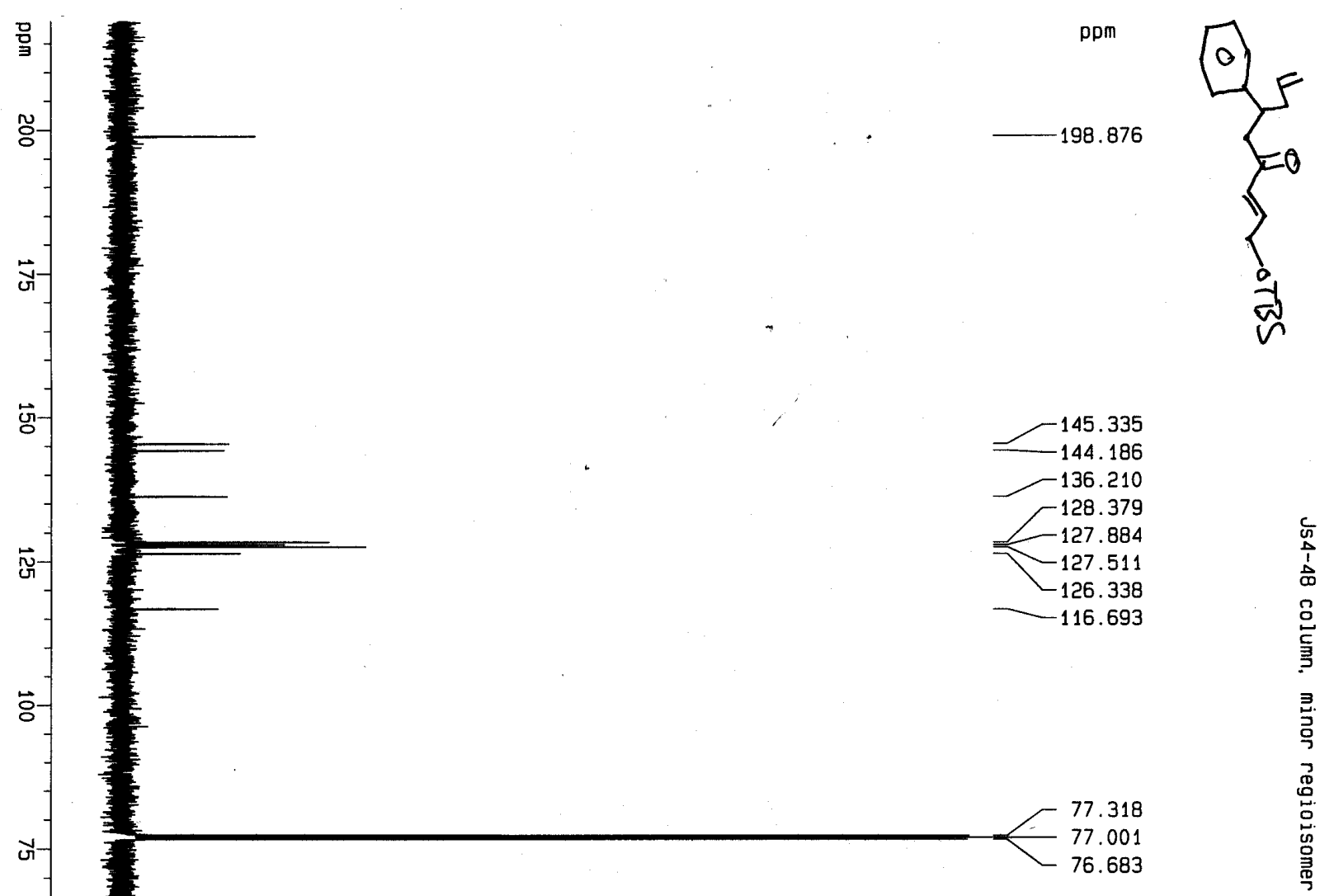

62.229

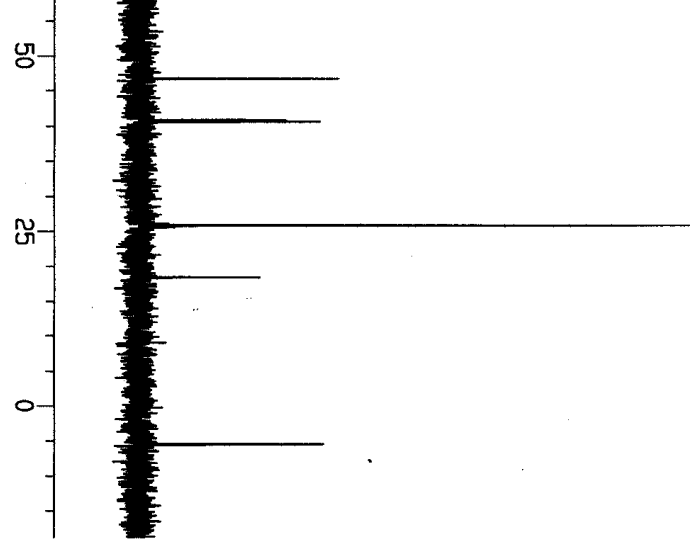

46.704

40.813

40.599

25.837

18. 346

$-5.446$

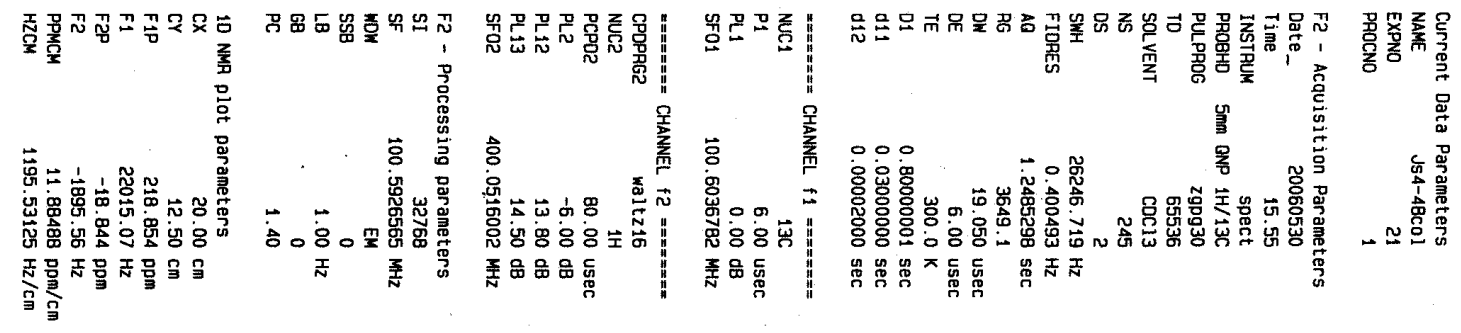




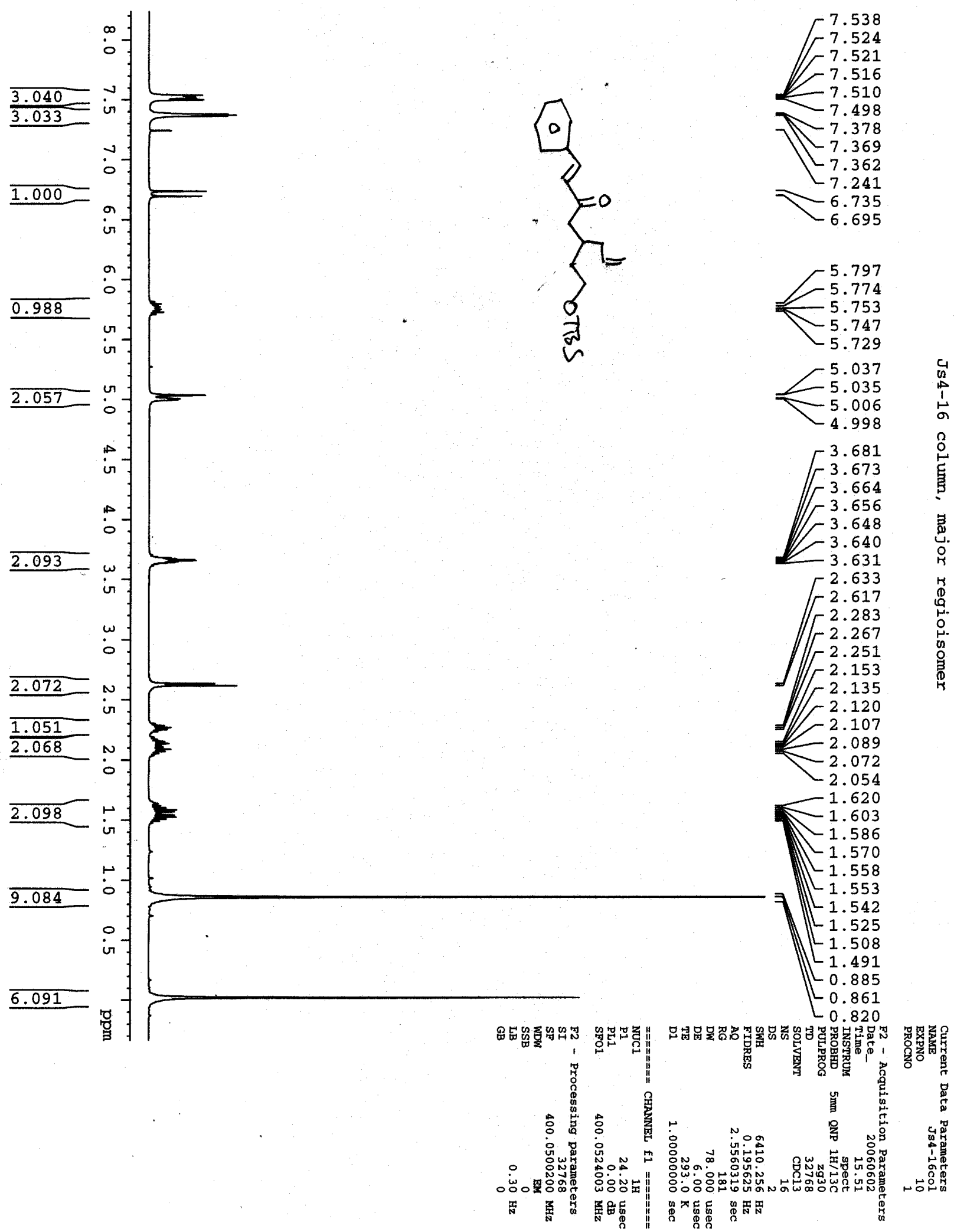




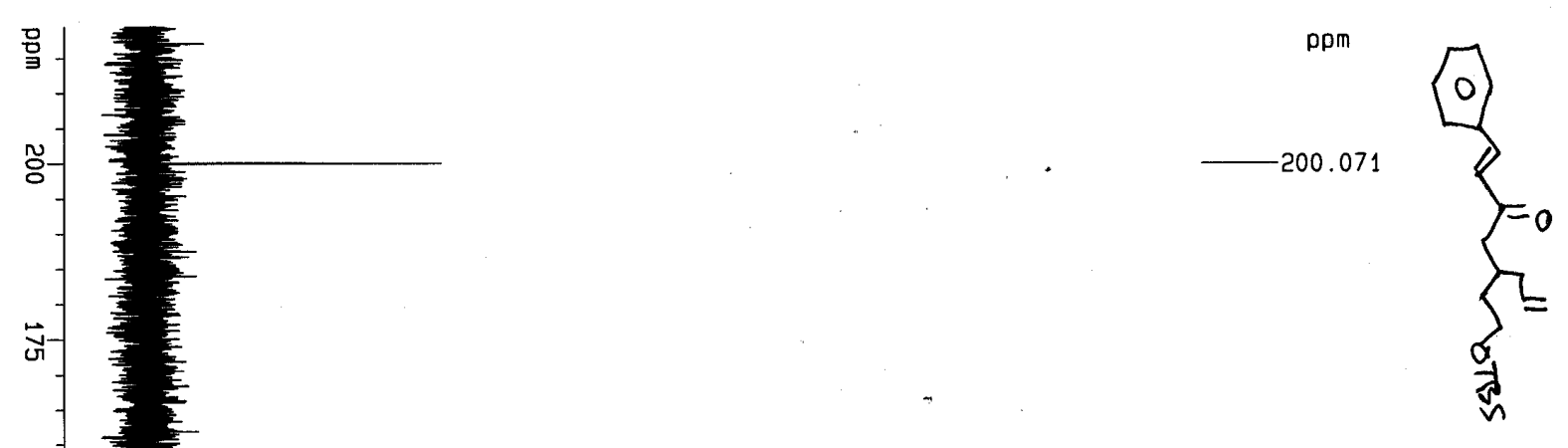

병-

G-

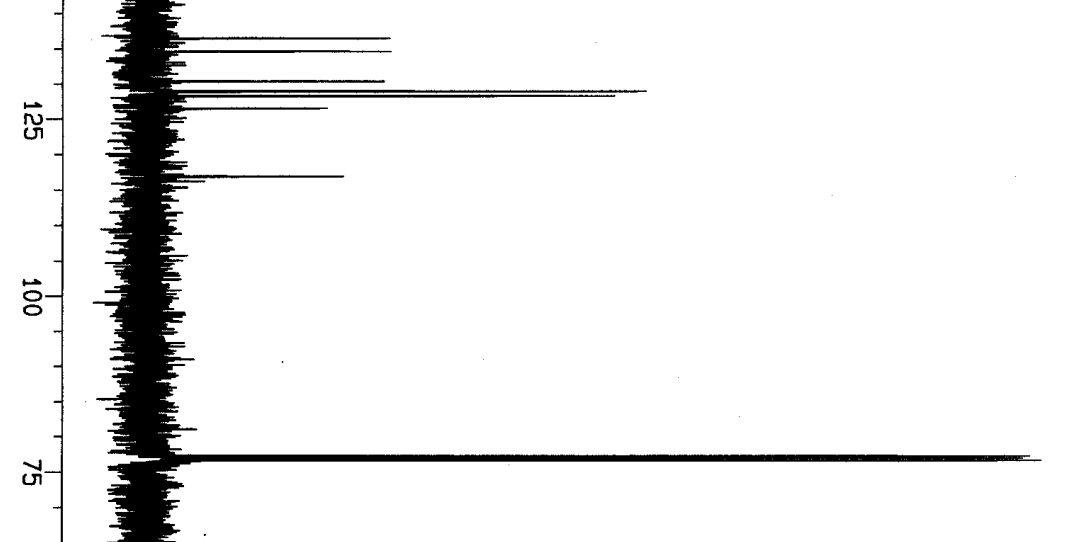

$-136.385$

$-130.348$

$-128.886$

$-128.229$

$-126.475$

$-116.857$

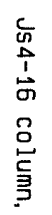

77.317

76.999

76.681

61.195

당-

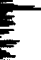

(1)

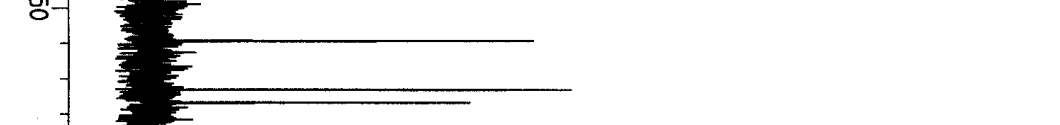

$-45.286$

$-38.422$

$-36.605$

31.228

ㄴ-

25.919

18.256

o-

$-1$
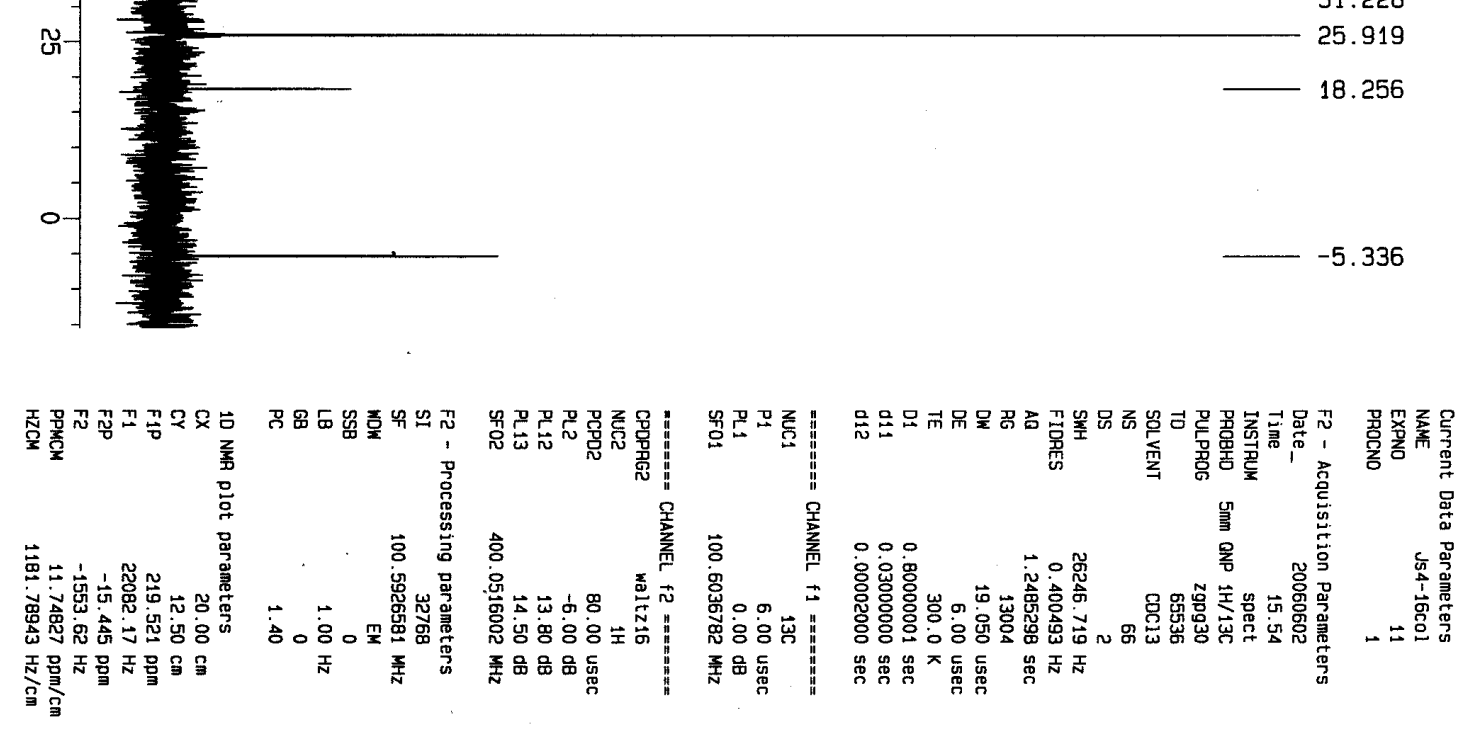


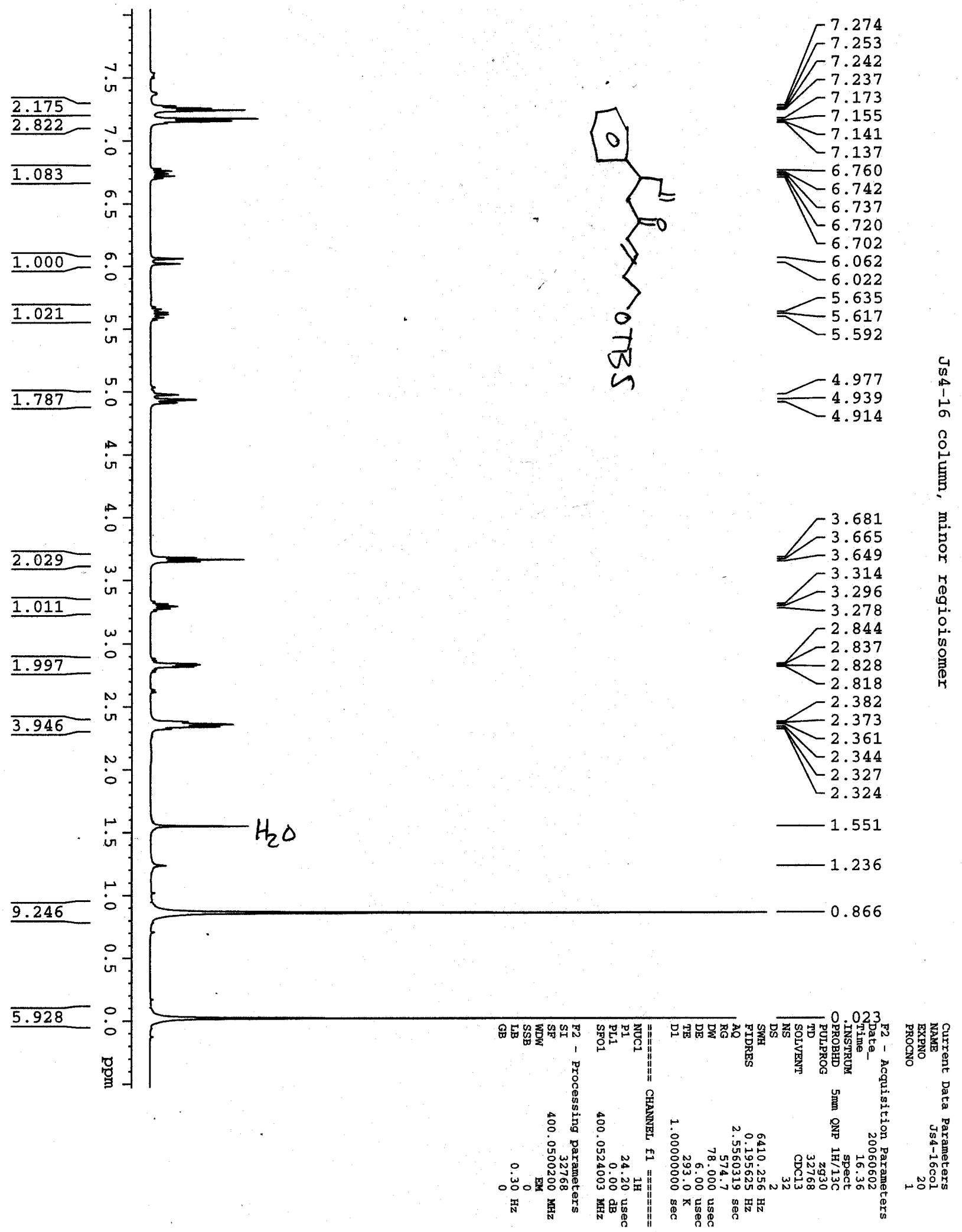




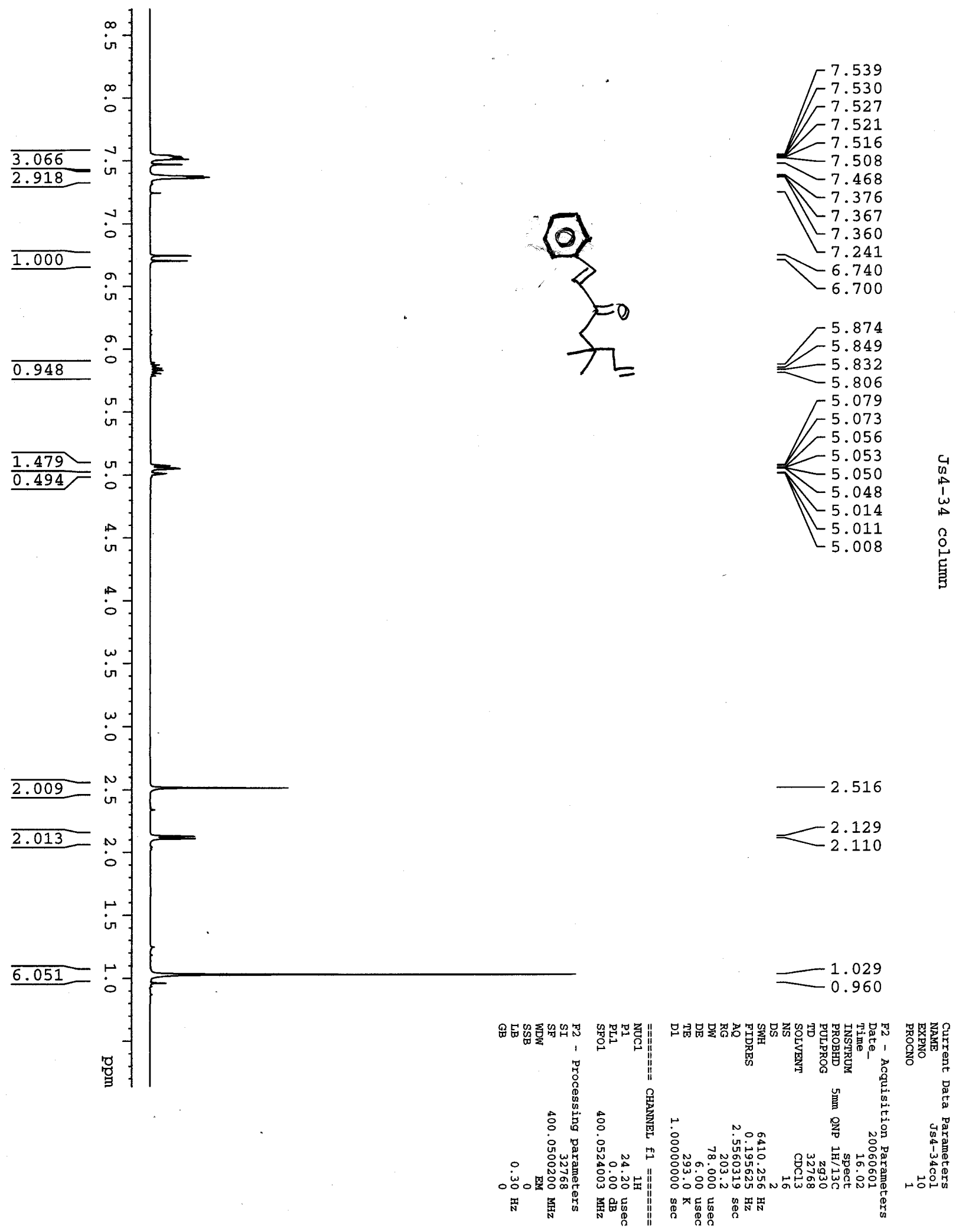



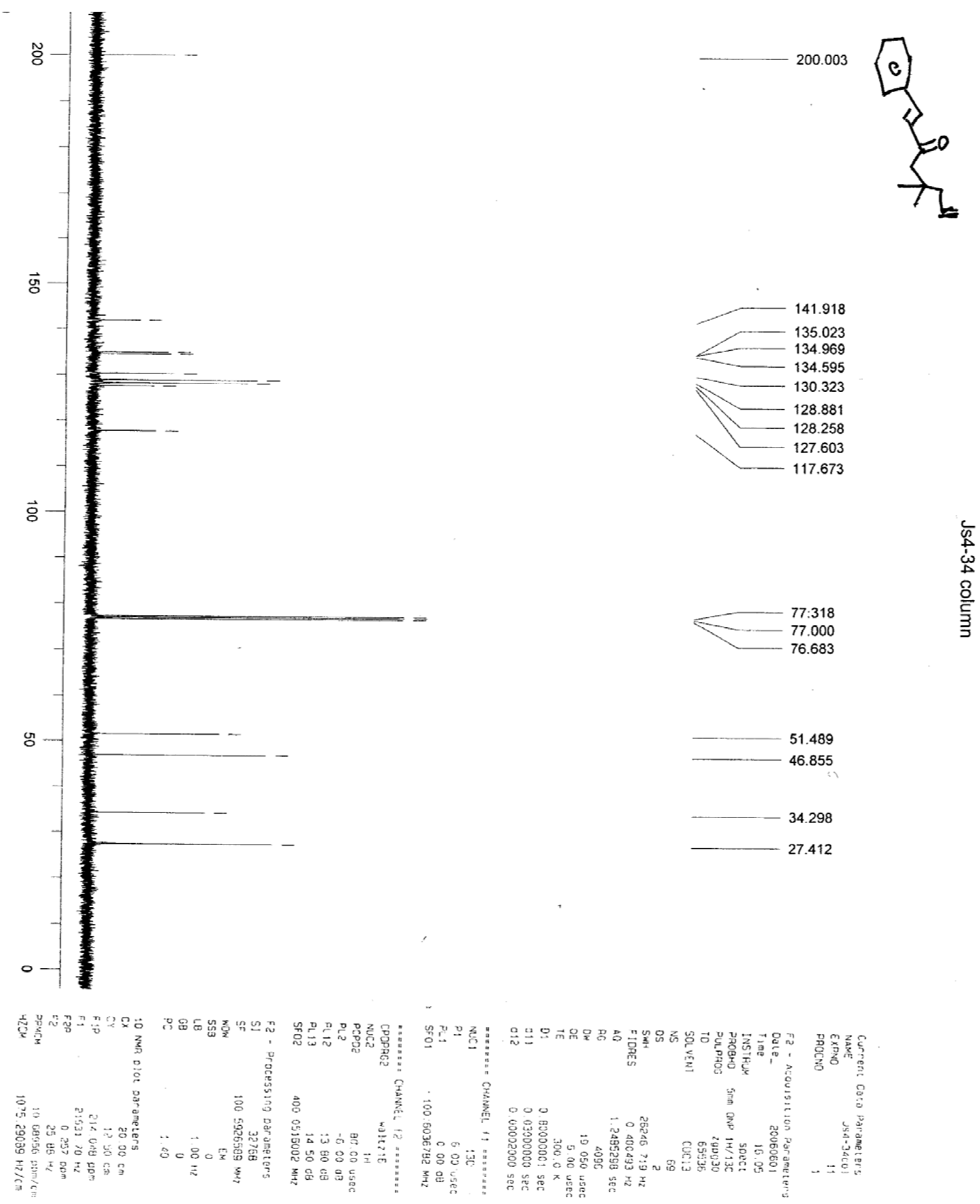

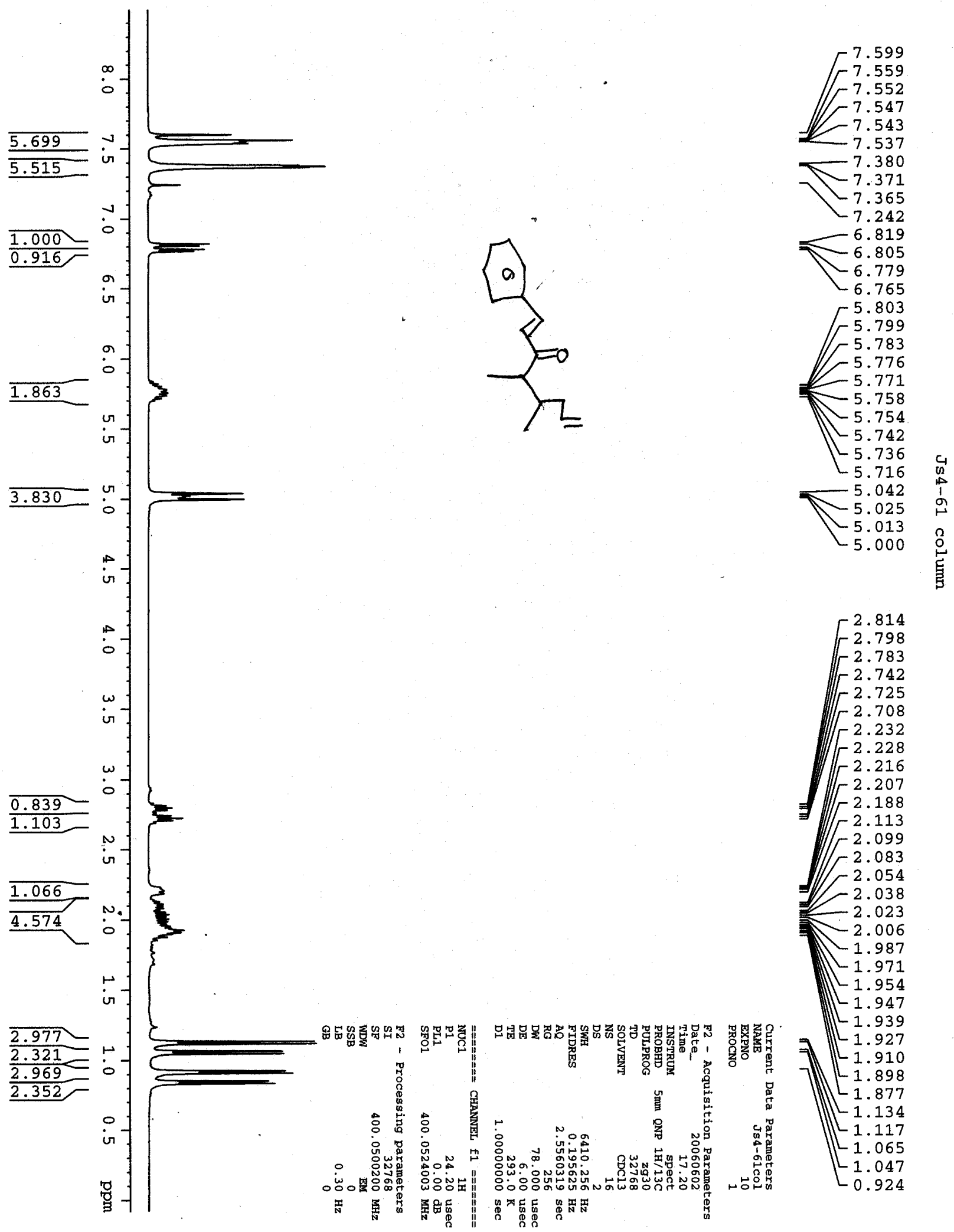

Page SI-48 

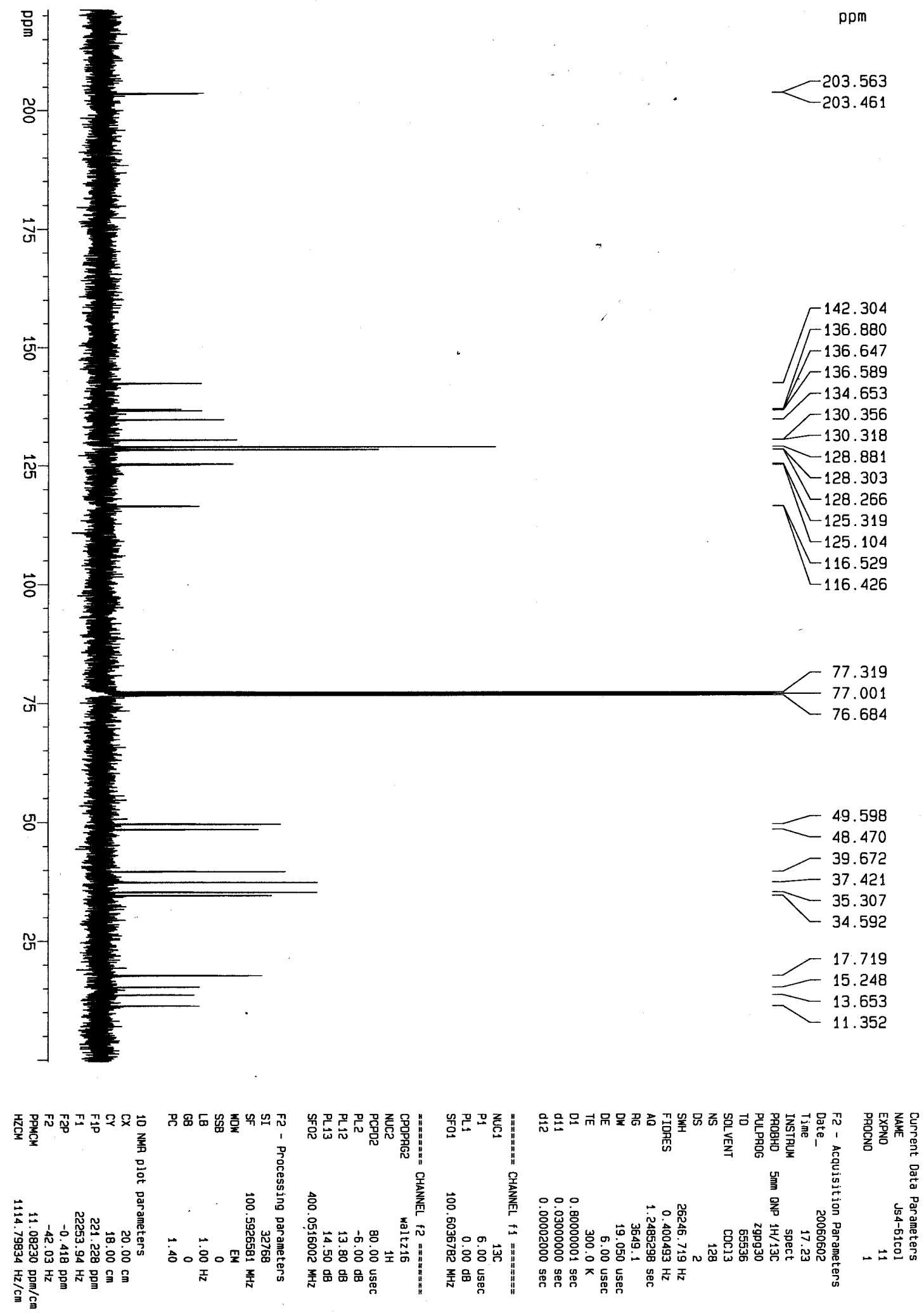


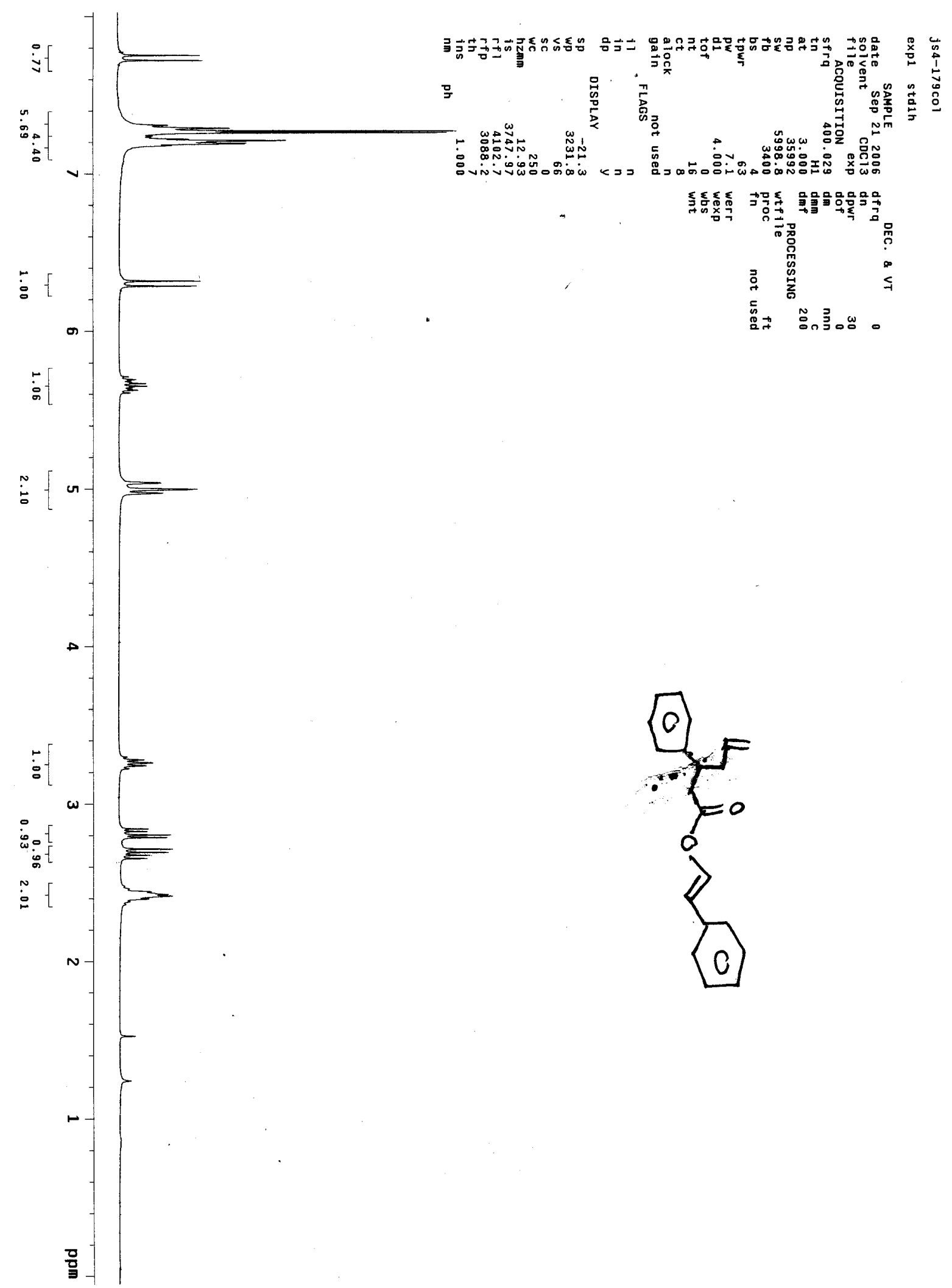

Page SI-50 


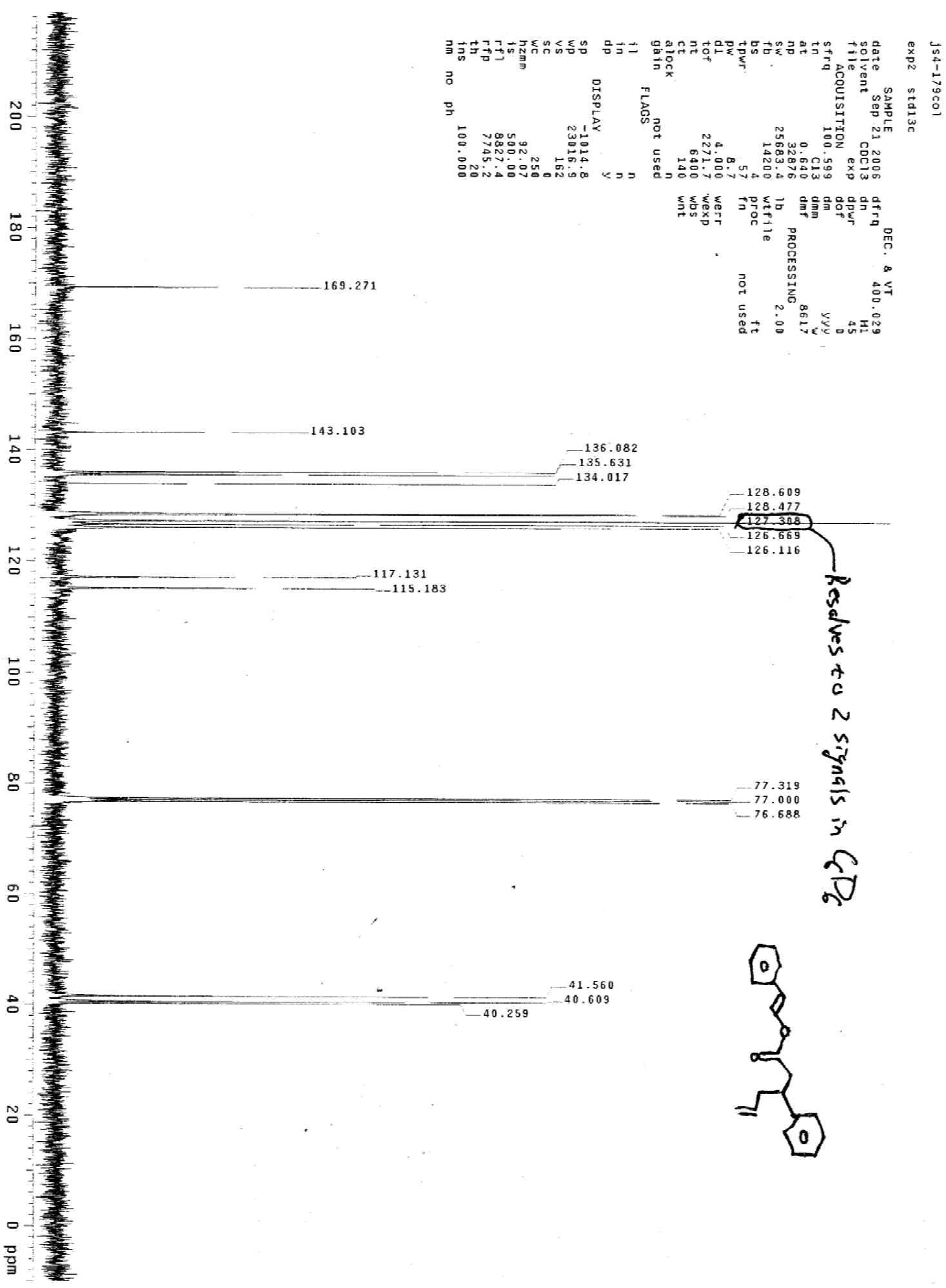




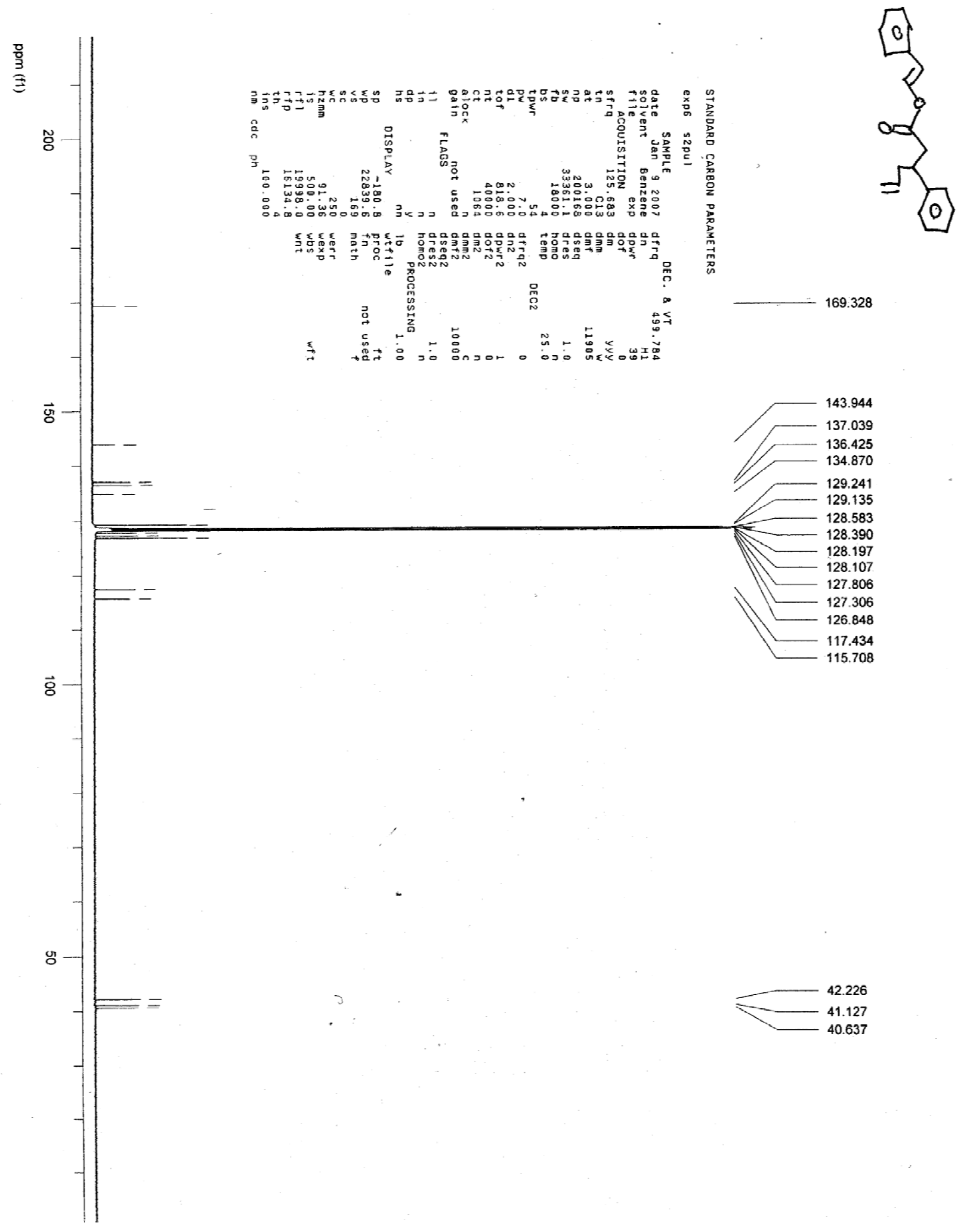

Page SI-52 